\title{
THE INVARIANT TRACE FORMULA. I. LOCAL THEORY
}

\author{
JAMES ARTHUR
}

\section{Contents}

Introduction

1. Invariant harmonic analysis

2. The invariant distributions $I_{M}(\gamma)$

3. The invariant distributions $I_{M}(\pi, X)$

4. Some further maps and distributions

5. A contour integral

6. Reduction of induction hypotheses

7. A property of $(G, M)$-families

8. Descent

9. Splitting

10. The example of $G L(n)$. Local vanishing properties

Appendix. Convex polytopes

\section{INTRODUCTION}

The well-known Poisson summation formula applies to a lattice $\Gamma$ in $\mathbb{R}$ and a function $f \in C_{c}^{\infty}(\mathbb{R})$. It can be written

$$
\sum_{\gamma \in \mathbf{R}} a^{\Gamma}(\gamma) f(\gamma)=\sum_{\lambda \in \mathbf{R}} \hat{a}^{\Gamma}(\lambda) \hat{f}(\lambda),
$$

where $\hat{f}$ is the Fourier transform of $f$, while

$$
a^{\Gamma}(\gamma)=\left\{\begin{array}{l}
\text { volume }(\mathbb{R} / \Gamma), \quad \text { if } \gamma \in \Gamma, \\
0, \quad \text { if } \gamma \notin \Gamma,
\end{array}\right.
$$

and

$$
\hat{a}^{\Gamma}(\lambda)= \begin{cases}1, & \text { if } \lambda \Gamma \subset \mathbb{Z}, \\ 0, & \text { otherwise. }\end{cases}
$$

Notice the general structure of the terms. The functions $f(\gamma)$ and $\hat{f}(\lambda)$ are independent of $\Gamma$, while the coefficients $a^{\Gamma}(\gamma)$ and $\hat{a}^{\Gamma}(\lambda)$ are independent of $f$. The Poisson summation formula has a number of applications. They all involve playing some of the terms off against the others.

Received by the editors January 5, 1987.

1980 Mathematics Subject Classification (1985 Revision). Primary 22E55. 
The Poisson summation formula has a generalization to a discrete subgroup of a general locally compact (unimodular) group with compact quotient. It is the Selberg trace formula. For example, suppose that $G / \mathbb{Q}$ is a semisimple algebraic group which is anisotropic. Then $G(\mathbb{Q})$ is a discrete subgroup of the locally compact group

$$
G(\mathbb{A})=G(\mathbb{R}) \times G\left(\mathbb{Q}_{2}\right) \times G\left(\mathbb{Q}_{3}\right) \times G\left(\mathbb{Q}_{5}\right) \times \cdots
$$

such that $G(\mathbb{Q}) \backslash G(\mathrm{~A})$ is compact. The Selberg trace formula is

$$
\sum_{\gamma \in(G(\mathbb{Q}))} a^{G}(\gamma) I_{G}(\gamma, f)=\sum_{\pi \in \Pi(G)} a^{G}(\pi) I_{G}(\pi, f), \quad f \in C_{c}^{\infty}(G(\mathrm{~A})),
$$

where $(G(\mathbb{Q}))$ is the set of conjugacy classes in $G(\mathbb{Q}), \Pi(G)$ is a set of (equivalence classes of) irreducible unitary representations of $G(\mathrm{~A})$, and

$$
\begin{gathered}
a^{G}(\gamma)=\operatorname{volume}(G(\mathbb{Q}, \gamma) \backslash G(\mathbf{A}, \gamma)), \\
a^{G}(\pi)=\operatorname{Multiplicity}\left(\pi, L^{2}(G(\mathbb{Q}) \backslash G(\mathrm{~A})),\right. \\
I_{G}(\gamma, f)=\int_{G(\mathbf{A}, \gamma) \backslash G(\mathbf{A})} f\left(x^{-1} \gamma x\right) d x, \\
I_{G}(\pi, f)=\operatorname{trace}\left(\int_{G(\mathbf{A})} f(x) \pi(x) d x\right) .
\end{gathered}
$$

Again, the terms have the same general structure. The functions $I_{G}(\gamma, f)$ and $I_{G}(\pi, f)$ are invariant distributions on $G(\mathrm{~A})$ which do not really depend on the discrete subgroup $G(\mathbb{Q})$. The coefficients $a^{G}(\gamma)$ and $a^{G}(\pi)$ depend strongly on $G(\mathbb{Q})$, but are independent of $f$. The Selberg trace formula also has many applications. Again, one obtains information about one set of terms from a knowledge of the others.

If $G / \mathbb{Q}$ is not anisotropic, the quotient $G(\mathbb{Q}) \backslash G(\mathrm{~A})$ is no longer compact, and the situation changes rather drastically. The terms in (2) diverge (in several senses) and are in general not defined. There are natural ways to truncate the integrals that diverge, however, and one ends up with a trace formula that appears quite complicated. In this paper and the next one [1(f)], we shall show that the general structure of the trace formula is rather simple. We shall establish an identity of the general form

$$
\begin{aligned}
& \sum_{M}\left|W_{0}^{M}\right|\left|W_{0}^{G}\right|^{-1} \sum_{\gamma \in(M(\mathbb{Q}))} a^{M}(\gamma) I_{M}(\gamma, f) \\
& \quad=\sum_{M}\left|W_{0}^{M}\right|\left|W_{0}^{G}\right|^{-1} \int_{\Pi(M)} a^{M}(\pi) I_{M}(\pi, f) d \pi,
\end{aligned}
$$

in which $M$ ranges over a finite set of rational Levi subgroups of $G$. The terms corresponding to $M \neq G$ represent contributions from the boundary. They are what is left of the original integrals that had to be truncated. The functions $a^{M}(\gamma)$ and $a^{M}(\pi)$ depend only on the group $M$ and not its embedding in $G$. They are global in nature, in that they depend on the rational structure 
of $M$. The functions $I_{M}(\gamma, f)$ and $I_{M}(\pi, f)$ are invariant linear forms in $f$. They are local objects which are essentially independent of the discrete subgroup $G(\mathbb{Q})$ of $G(\mathrm{~A})$. The applications of the general trace formula are only beginning. If they follow the pattern of $G L(2)$, one will be able to deduce information about the discrete spectrum, which is a priori wrapped up in the definition of the function $a^{G}(\pi)$, from the other terms in the trace formula.

We shall leave the global theory of (3) and the proof of the formula itself for the next paper [1(f)]. In this paper, we shall study the functions $I_{M}(\gamma, f)$ and $I_{M}(\pi, f)$. These are interesting objects in their own right. If $M=G$, $I_{M}(\gamma, f)$ is just the orbital integral over $\gamma$ and $I_{M}(\pi, f)$ is the character of $\pi$. For general $M$ they are more complicated, but they retain many of the essential properties of the special case.

It is best to take $G$ to be a connected reductive group over a number field $F$. If $S$ is a finite set of valuations of $F$, one can define

$$
I_{M}(\gamma, f), \quad \gamma \in M\left(F_{S}\right),
$$

and

$$
I_{M}(\pi, f), \quad \pi \in \Pi_{\text {unit }}\left(M\left(F_{S}\right)\right),
$$

as invariant linear forms on the Hecke algebra of $G\left(F_{S}\right)$. It is important to express them in terms of the local groups $G\left(F_{v}\right)$. In $\S 9$, we shall prove splitting formulas for $I_{M}(\gamma, f)$ and $I_{M}(\pi, f)$ in terms of the corresponding objects on the groups $G\left(F_{v}\right), v \in S$. A related question concerns the case that the data $\gamma$ and $\pi$ come from a proper Levi subgroup $M_{1}$ of $M$. In $\S 8$ we shall prove descent formulas for $I_{M}(\gamma, f)$ and $I_{M}(\pi, f)$ in terms of the corresponding objects for $M_{1}$. Both sets of results will be proved from Proposition 7.1, which gives a general descent property for $(G, M)$-families. This in turn is closely related to a similar property for convex polytopes, which we will leave for the appendix.

It is perhaps helpful to think of the distributions $I_{M}(\gamma, f)$ and $I_{M}(\pi, f)$ themselves in terms of convex polytopes. Indeed, the chambers of the restricted Weyl group are dual to a certain convex polytope $\Pi_{0}$. The groups $M$ are parametrized by subspaces which intersect faces of $\Pi_{0}$ orthogonally. If we project $\Pi_{0}$ onto such a subspace, we obtain another convex polytope $\Pi_{M}$. The geometry of $\Pi_{M}$ then governs the descent and splitting properties of the corresponding distributions.

The invariant distributions $I_{M}(\gamma, f)$ are obtained from the weighted orbital integrals $J_{M}(\gamma, f)$ studied in [1(d)]. In $\S 2$ we shall list the various properties that $I_{M}(\gamma, f)$ inherits from $J_{M}(\gamma, f)$. They all generalize well-known properties of ordinary orbital integrals. For example, the value of $I_{M}(\gamma, f)$ at a general point $\gamma \in M\left(F_{S}\right)$ can be approximated by its values at $G$-regular points in $M\left(F_{S}\right)$. If $S$ consists of one Archimedean valuation, $I_{M}(\gamma, f)$ satisfies a differential equation in $\gamma$. It also has a simple formula for the jump across the singular hyperplane of a real root. If $S$ consists of one discrete valuation, $I_{M}(\gamma, f)$ satisfies a germ expansion in $\gamma$. 
The distributions $I_{M}(\pi, f)$ are the values at $X=0$ (and $\pi$ unitary) of a more general family of invariant distributions

$$
I_{M}(\pi, X, f), \quad \pi \in \Pi\left(M\left(F_{S}\right)\right), X \in \mathfrak{a}_{M, S},
$$

which we introduce in $\S 3$. These are defined in terms of the weighted characters

$$
J_{M}(\pi, X, f)=\int_{i \mathfrak{a}_{M} . S} J_{M}\left(\pi_{\lambda}, f\right) e^{-\lambda(X)} d \lambda,
$$

studied in [1(e)]. It will follow from the definition that $I_{M}(\pi, X, f)$ is trivial if $\pi$ is tempered (Lemma 3.1). However, for general $\pi$, the distribution is more interesting. It turns out to be closely related to the residues (in $\pi_{\lambda}$ ) of $J_{M}\left(\pi_{\lambda}, f\right)$. There are hints of this in Lemmas 3.2 and 3.3, but a full explanation will have to await another paper.

It happens that the distributions $I_{M}(\gamma)$ and $I_{M}(\pi, X)$ are not independent of each other. This is fortunate because it enhances the possibility of playing them off against each other in the trace formula. If $\gamma$ is restricted to a maximal torus $T\left(F_{S}\right)$ in $M\left(F_{S}\right)$, the weighted orbital integral $J_{M}(\gamma, f)$ is compactly supported in $\gamma$. However, it turns out that $I_{M}(\gamma, f)$ is not compactly supported in $\gamma$. The distributions $I_{M}(\pi, X, f)$ may be viewed as the obstruction to this. In $\S 4$ we shall study various objects which arise naturally when one tries to analyze the asymptotic behavior of $I_{M}(\gamma, f)$. We shall define new invariant distributions ${ }^{c} I_{M}(\gamma, f)$ and ${ }^{c} I_{M}(\pi, X, f)$ by improving the support properties at the expense of properties of smoothness. In particular, we shall show that ${ }^{c} I_{M}(\gamma, f)$ is compactly supported if $\gamma$ lies in $T\left(F_{S}\right)$ (Lemma 4.4.). We shall also define certain maps $\theta_{M}$ and ${ }^{c} \theta_{M}$ that provide expansions for $I_{M}$ and ${ }^{c} I_{M}$ in terms of each other. These maps are in fact determined by the asymptotic behavior of $I_{M}(\gamma, f)$. This sets the stage for Proposition 5.4. The result is an important formula for ${ }^{c} I_{M}(\pi, X, f)$ as a contour integral involving ${ }^{c} \theta_{M}(f)$. It follows that the distributions ${ }^{c} I_{M}(\pi, X, f)$ and $I(\pi, X, f)$ may be determined, at least in principle, from the asymptotic behavior of $I_{M}(\gamma, f)$.

In $\S 6$ we shall give a simple example of how Proposition 5.4 can be applied in practice. It is not known in general that an invariant distribution annihilates functions whose orbital integrals vanish. In Theorem 6.1 we shall show that this property holds for $I_{M}(\pi, X)$ provided that it holds for $I_{M}(\gamma)$. (We will establish the property for $I_{M}(\gamma)$ in the next paper [1(f)].)

We have already mentioned the descent and splitting formulas that are proved in $§ \S 7-9$. To illustrate the descent formulas, we shall end the paper by discussing the example of $G L(n)$. We shall show that our invariant distributions often vanish on functions associated with base change or the comparison with central simple algebras. These vanishing formulas (Propositions 10.2 and 10.3) will in fact be required for base change. Together with global vanishing results in $[1(f), \S 8]$, they are the starting point for a comparison of the full trace formula of $G L(n)$ with the twisted trace formula over a cyclic extension. 


\section{INVARIANT HARMONIC ANALYSIS}

Let $G$ be a connected component of a reductive algebraic group over a field $F$. We assume that $G(F) \neq \varnothing$. We write $G^{+}$for the group generated by $G$, and $G^{0}$ for the identity component of $G^{+}$. A simple example to keep in mind is the component

$$
G^{*}=\underbrace{(G L(n) \times \cdots \times G L(n))}_{l} \rtimes \theta^{*},
$$

when $\theta^{*}$ is the permutation

$$
(1, \ldots, l) \rightarrow(2, \ldots, l, 1) .
$$

Then $\left(G^{*}\right)^{+}$is the semidirect product of $l$ copies of $G L(n)$ with the cyclic group of order $l$ generated by $\theta^{*}$. A more general example is that in which $G$ is an inner twist of $G^{*}$. By this we mean that there is a morphism

$$
\eta: G \rightarrow G^{*} \text {, }
$$

which extends to an isomorphism $\eta$ from $G^{+}$to $\left(G^{*}\right)^{+}$, such that for every $\tau \in \operatorname{Gal}(\bar{F} / F), \eta^{-1} \eta^{\tau}$ equals a conjugation by an element in $G^{+}$. If $G$ is of this form it is essentially the connected component obtained from a central simple algebra by cyclic base change.

We assume that $F$ is a local or a global field of characteristic 0 . In this paper, $S$ always stands for a finite set of valuations of $F$ with the closure property [1(e), §1]. This simply means that if $S$ contains no Archimedean valuations, it consists entirely of valuations which divide a fixed rational prime $p$. We fix a maximal compact subgroup

$$
K^{+}=\prod_{v \in S} K_{v}^{+}
$$

of $G^{+}\left(F_{S}\right)$, such that the group

$$
K_{v}=K_{v}^{+} \cap G^{0}\left(F_{v}\right)
$$

is special for every non-Archimedean valuation $v \in S$. Clearly,

$$
K=\prod_{v \in S} K_{v}
$$

is a maximal compact subgroup of $G^{0}\left(F_{S}\right)$. Having fixed $K$, we can form the Hecke space $\mathscr{H}\left(G\left(F_{S}\right)\right.$ ). It consists of the smooth, compactly supported functions on $G\left(F_{S}\right)$ which are left and right $K$-finite.

The Hecke space seems to be the correct space of test functions to use in the trace formula. We are interested in the continuous linear functionals or "distributions" on $\mathscr{H}\left(G\left(F_{S}\right)\right)$ which make up the individual terms in the trace formula. In the papers [1(d)] and [1(e)], we studied the local properties of two such families of distributions. The present article is a natural successor to $[1(d)]$ and $[1(e)]$ and in a sense unites these previous two papers. We shall 
attach invariant distributions to each of the distributions in the two families. By studying the parallel behavior of these, we shall find that the two families are really quite closely related.

We shall routinely adopt the notation of [1(d)] and [1(e)], especially that of $\S 1$ of each paper. In particular, the letter $M$ is always understood to be a Levi subset of $G$ which is in good relative position with respect to $K$. More precisely, we require that each $K_{v}$ be admissible relative to $M^{0}$ in the sense of $\S 1$ of [1(a)]. Recall that $\mathscr{L}(M)$ denotes the collection of Levi subsets of $G$ which contain $M$, and $\mathscr{F}(M)$ denotes the set of parabolic subsets

$$
P=M_{P} N_{P}, \quad M_{P} \in \mathscr{L}(M),
$$

which contain $M$. Recall also that we have the real vector space

$$
\mathfrak{a}_{M}=\operatorname{Hom}\left(X(M)_{F}, \mathbb{R}\right),
$$

which we assume has been assigned a suitable Euclidean metric. This provides a Euclidean metric by restriction on any subspace of $\mathfrak{a}_{M}$.

In $\S 11$ of $[1(\mathrm{e})]$ we defined the Paley-Wiener space $\mathscr{I}\left(G\left(F_{S}\right)\right)$ of functions on

$$
\Pi_{\text {temp }}\left(G\left(F_{S}\right)\right) \times \mathfrak{a}_{G, S} .
$$

(Recall that $\mathfrak{a}_{G, S}$ is the subgroup $H_{G}\left(G^{+}\left(F_{S}\right)\right)$ of $\mathfrak{a}_{G}$.) There is a continuous map

$$
\mathscr{T}: f \rightarrow f_{G}, \quad f \in \mathscr{H}\left(G\left(F_{S}\right)\right),
$$

with

$$
f_{G}(\pi, X)=\int_{\mathfrak{i a}_{G . S}^{*}} \operatorname{tr}\left(\pi_{\lambda}(f)\right) e^{-\lambda(X)} d \lambda, \quad \pi \in \Pi_{\mathrm{temp}}\left(G\left(F_{S}\right)\right), X \in \mathfrak{a}_{G, S},
$$

from $\mathscr{H}\left(G\left(F_{S}\right)\right)$ to $\mathscr{I}\left(G\left(F_{S}\right)\right)$. More generally, consider the function

$$
\begin{aligned}
f_{M}(\pi, X)=\left(f_{P}\right)_{M}(\pi, X)=\int_{i a_{M}^{*} S} \operatorname{tr}\left(\mathscr{I}_{P}\left(\pi_{\lambda}, f\right)\right) e^{-\lambda(X)} d \lambda, \\
\quad \pi \in \Pi_{\text {temp }}\left(M\left(F_{S}\right)\right), \quad X \in \mathfrak{a}_{M, S},
\end{aligned}
$$

where for any $P \in \mathscr{P}(M), f_{P}$ is the function

$$
m \rightarrow \delta_{P}(m)^{1 / 2} \int_{K} \int_{N_{P}\left(F_{S}\right)} f\left(k^{-1} m n k\right) d n d k
$$

in $\mathscr{H}\left(M\left(F_{S}\right)\right)$, and $\mathscr{F}_{P}\left(\pi_{\lambda}\right)$ is the representation in $\Pi_{\text {temp }}\left(G\left(F_{S}\right)\right)$ induced from $\pi_{\lambda}$. Then $f \rightarrow f_{M}$ is a continuous linear map from $\mathscr{H}\left(G\left(F_{S}\right)\right)$ to $\mathscr{I}\left(M\left(F_{S}\right)\right)$.

It is actually necessary to work with the larger spaces

$$
\mathscr{H}_{\text {ac }}\left(G\left(F_{S}\right)\right)=\underset{\Gamma}{\lim } \mathscr{H}_{\text {ac }}\left(G\left(F_{S}\right)\right)_{\Gamma}
$$

and

$$
\mathscr{I}_{\mathrm{ac}}\left(G\left(F_{S}\right)\right)=\underset{\Gamma}{\lim } \mathscr{I}_{\mathrm{ac}}\left(G\left(F_{S}\right)\right)_{\Gamma}
$$


introduced also in $\S 11$ of [1(e)]. (Recall that $\Gamma$ denotes a finite subset of $\Pi(K)$, and $\mathscr{H}_{\mathrm{ac}}\left(G\left(F_{S}\right)\right)_{\Gamma}$ is the space of functions $f$ on $G\left(F_{S}\right)$ such that for any $b \in C_{c}^{\infty}\left(\mathfrak{a}_{G, S}\right)$, the function

$$
f^{b}(x)=f(x) b\left(H_{G}(x)\right)
$$

belongs to $\mathscr{H}\left(G\left(F_{S}\right)\right)_{\Gamma}$. Similarly, $\mathscr{F}_{\text {ac }}\left(G\left(F_{S}\right)\right)_{\Gamma}$ is the space of functions $\phi$ on $\Pi_{\text {temp }}\left(G\left(F_{S}\right)\right) \times \mathfrak{a}_{G, S}$ such that for every $b$, the function

$$
\phi^{b}(\pi, X)=\phi(\pi, X) b(X)
$$

belongs to $\left.\mathscr{I}\left(G\left(F_{S}\right)\right)_{\Gamma}\right)$. For there is an important map $\phi_{M}$ which sends $\mathscr{H}\left(G\left(F_{S}\right)\right)$ to a space of functions on

$$
\Pi_{\text {temp }}\left(M\left(F_{S}\right)\right) \times \mathfrak{a}_{M, S}
$$

which is not contained in $\mathscr{I}\left(M\left(F_{S}\right)\right)$. However, $\phi_{M}$ can be defined on $\mathscr{H}_{\mathrm{ac}}\left(G\left(F_{S}\right)\right)$, and it does map this space into $\mathscr{I}_{\mathrm{ac}}\left(M\left(F_{S}\right)\right)$ [1(e), Corollary 12.2]. Moreover, it follows directly from the definition that $f \rightarrow f_{M}$ extends to a continuous map from $\mathscr{H}_{\mathrm{ac}}\left(G\left(F_{S}\right)\right)$ into $\mathscr{I}_{\mathrm{ac}}\left(M\left(F_{S}\right)\right)$. In particular,

$$
\mathscr{T}: f \rightarrow f_{G}, \quad f \in \mathscr{H}_{\mathrm{ac}}\left(G\left(F_{S}\right)\right),
$$

maps $\mathscr{H}_{\mathrm{ac}}\left(G\left(F_{S}\right)\right)$ continuously into $\mathscr{I}_{\mathrm{ac}}\left(G\left(F_{S}\right)\right)$.

Proposition 1.1. Suppose that $G$ either equals $G^{0}$ or is an inner twist of the component $G^{*}$ in (1.1). Then

$$
\mathscr{T}: f \rightarrow f_{G}, \quad f \in \mathscr{H}_{\mathrm{ac}}\left(G\left(F_{S}\right)\right),
$$

is an open, surjective map from $\mathscr{H}_{\mathrm{ac}}\left(G\left(F_{S}\right)\right)$ onto $\mathscr{I}_{\mathrm{ac}}\left(G\left(F_{S}\right)\right)$.

Proof. It is enough to establish the result with the spaces $\mathscr{H}_{\text {ac }}\left(G\left(F_{S}\right)\right)$ and $\mathscr{F}_{\text {ac }}\left(G\left(F_{S}\right)\right)$ replaced by $\mathscr{H}\left(G\left(F_{S}\right)\right)$ and $\mathscr{I}\left(G\left(F_{S}\right)\right)$. Indeed, the topologies on the larger spaces are defined so that the openness assertion extends immediately. One extends the surjectivity to the larger spaces by a partition of unity argument on $\mathfrak{a}_{G, S}$. It is also clear that the valuations in $S$ may be treated separately. We shall therefore assume that $S$ consists of one valuation, $\{v\}$, and that $F$ is a local field. Then $F_{S}=F_{v}=F$.

Suppose first that $F$ is non-Archimedean. The surjectivity of the map $\mathscr{H}(G(F)) \rightarrow \mathscr{I}(G(F))$ follows directly from the trace Paley-Wiener theorem of Bernstein, Deligne and Kazhdan [3], and its extension to nonconnected groups by Rogawski [8]. It holds without restriction on $G$. The openness is trivial since $\mathscr{H}(G(F))$ and $\mathscr{I}(G(F))$ are topological direct limits of finite dimensional spaces.

Suppose next that $F$ is Archimedean. In the case that $G=G^{0}$, the surjectivity has been proved by Clozel and Delorme ([5(a)], [5(b)]). In [5(b)], the authors note that the theorem can be claimed only for connected Lie groups. However, the results of Knapp and Zuckerman, which were the reason for the restriction, are known to hold in general [9]. The openness assertion can also 
be extracted from the work of Clozel and Delorme. For implicit in their proof of surjectivity is the construction of a continuous section

$$
\mathscr{I}\left(G\left(F_{S}\right)\right) \rightarrow \mathscr{H}\left(G\left(F_{S}\right)\right) .
$$

(See the appendix to [1(f)].) If $G$ is an inner twist of $G^{*}$, the trace PaleyWiener theorem can be proved in the same way as for connected groups. For the special case of base change for $G L(n)$, see [2, Lemma I.7.1]. The more general case follows the same way. Again, the openness of the map is implicit in the proof of its surjectivity.

For the rest of this paper and also the next one [1(f)], we shall assume that $G$ satisfies the conditions of Proposition 1.1. That is, $G$ equals $G^{0}$ or $G$ is an inner twist of the component $G^{*}$ in (1.1). This is only because of the limitations of Proposition 1.1. We shall, in fact, write the papers as if they applied to general $G$. In the next paper, there will be one argument in Galois cohomology that relies on the special nature of $G$ [1(f), Theorem 5.1]. However, it seems likely that both this argument and Proposition 1.1 could soon be strengthened to include all $G$. The results of our two papers would then apply without restriction.

Suppose that $\theta$ is a continuous linear map from $\mathscr{H}_{\mathrm{ac}}\left(G\left(F_{S}\right)\right)$ to another topological vector space $\mathscr{V}$. We shall say that $\theta$ is supported on characters if it vanishes on the kernel of $\mathscr{T}$. That is, if $\theta(f)=0$ for every function $f \in \mathscr{H}_{\text {ac }}\left(G\left(F_{S}\right)\right)$ such that $f_{G}=0$. If $\theta$ has this property, there is a unique continuous map

$$
\hat{\theta}: \mathscr{I}_{\mathrm{ac}}\left(G\left(F_{S}\right)\right) \rightarrow \mathscr{V}
$$

such that

$$
\hat{\theta}\left(f_{G}\right)=\theta(f), \quad f \in \mathscr{H}_{\mathrm{ac}}\left(G\left(F_{S}\right)\right) .
$$

This is an immediate consequence of Proposition 1.1. Consider the special case that $\mathscr{V}=\mathbb{C}$. Then $\theta$ is supported on characters if and only if it lies in the image of the transpose map

$$
\mathscr{T}^{\prime}: \mathscr{F}_{\mathrm{ac}}^{\prime}\left(G\left(F_{S}\right)\right) \rightarrow \mathscr{H}_{\mathrm{ac}}^{\prime}\left(G\left(F_{S}\right)\right) .
$$

The function $\hat{\theta}$ is then just equal to the inverse image of $\theta$ under $\mathscr{T}^{\prime}$. As in [1(e)], we shall often refer to elements in the dual spaces $\mathscr{H}_{\text {ac }}^{\prime}\left(G\left(F_{S}\right)\right)$ and $\mathscr{F}_{\mathrm{ac}}^{\prime}\left(G\left(F_{S}\right)\right)$ as distributions on $\mathscr{H}_{\mathrm{ac}}\left(G\left(F_{S}\right)\right)$ and $\mathscr{F}_{\mathrm{ac}}\left(G\left(F_{S}\right)\right)$.

Any map

$$
\theta: \mathscr{H}_{\text {ac }}\left(G\left(F_{S}\right)\right) \rightarrow \mathscr{V}
$$

which is supported on characters is also invariant. That is,

$$
\theta\left(L_{h} f\right)=\theta\left(R_{h} f\right), \quad h \in \mathscr{H}\left(G^{0}\left(F_{S}\right)^{1}\right), f \in \mathscr{H}_{\mathrm{ac}}\left(G\left(F_{S}\right)\right),
$$

in the notation of $\S 6$ of [1(e)]. Conversely, it is likely that every map which is invariant is supported on characters. However, we shall not try to prove this. We shall be content simply to show that those invariant maps and distributions 
which arise from the trace formula are supported on characters. The proof will be based on a long induction and will not be completed until the next paper [1(f), Corollary 5.3], where we will use a global argument introduced by Kazhdan. The proof does not require that we keep track of which maps are invariant. However, we shall do so, in order to motivate our constructions. In fact, the reader might find it easier to proceed as if it were known that all invariant maps were supported on characters.

\section{The INVARIANt Distributions $I_{M}(\gamma)$}

We shall introduce one of the two families of invariant distributions which occur in the trace formula. These distributions are parametrized by elements in $M\left(F_{S}\right)$ and are obtained from weighted orbital integrals. They were defined in $\S 10$ of [1(a)] in the special case that $G=G^{0}$ and the element in $M\left(F_{S}\right)$ was $G$-regular. The definitions of [1(a)] relied on various hypotheses from local harmonic analysis.

Suppose that $\gamma$ is an element in $M\left(F_{S}\right)$. In $\S 6$ of [1(d)] we defined the weighted orbital integral

$$
J_{M}(\gamma, f), \quad f \in C_{c}^{\infty}\left(G\left(F_{S}\right)\right) .
$$

It is a distribution which depends only on the restriction of $f$ to

$$
G\left(F_{S}\right)^{Z}=\left\{x \in G\left(F_{S}\right): H_{G}(x)=Z\right\},
$$

for $Z=H_{G}(\gamma)$. The restriction of any function in $\mathscr{H}_{\mathrm{ac}}\left(G\left(F_{S}\right)\right)$ to this set coincides with that of a function in $\mathscr{H}\left(G\left(F_{S}\right)\right)$. Consequently, $J_{M}(\gamma)$ may be regarded as a distribution on $\mathscr{H}_{\mathrm{ac}}\left(G\left(F_{S}\right)\right)$. Arguing as in the proof of Lemma 6.2 of [1(e)], we can transform the formula

$$
J_{M}\left(\gamma, f^{y}\right)=\sum_{Q \in \mathscr{F}(M)} J_{M}^{M_{Q}}\left(\gamma, f_{Q, y}\right), \quad f \in C_{c}^{\infty}\left(G\left(F_{S}\right)\right),
$$

established in Lemma 8.1 of [1(a)], into

$$
J_{M}\left(\gamma, L_{h} f\right)=\sum_{Q \in \mathscr{F}(M)} J_{M}^{M_{Q}}\left(\gamma, R_{Q, h} f\right), \quad h \in \mathscr{H}_{\mathrm{ac}}\left(G^{0}\left(F_{S}\right)^{1}\right) .
$$

A similar formula,

$$
\phi_{M}\left(L_{h} f\right)=\sum_{Q \in \mathscr{F}(M)} \phi_{M}^{M_{Q}}\left(R_{Q, h} f\right)
$$

holds for the map

$$
\phi_{M}: \mathscr{H}_{\mathrm{ac}}\left(G\left(F_{S}\right)\right) \rightarrow \mathscr{F}_{\mathrm{ac}}\left(M\left(F_{S}\right)\right) .
$$

(See [1(e), (12.2)].) This suggests that we define an invariant distribution

$$
I_{M}(\gamma, f)=I_{M}^{G}(\gamma, f), \quad f \in \mathscr{H}_{\mathrm{ac}}\left(G\left(F_{S}\right)\right),
$$

inductively by setting

$$
J_{M}(\gamma, f)=\sum_{L \in \mathscr{L}(M)} \widehat{I}_{M}^{L}\left(\gamma, \phi_{L}(f)\right), \quad f \in \mathscr{H}_{\mathrm{ac}}\left(G\left(F_{S}\right)\right) .
$$


However, we cannot say that $I_{M}(\gamma)$ is supported on characters, so we do not know that $\widehat{I}_{M}(\gamma)$ is defined. We must proceed as follows. Let $\mathscr{L}_{0}(M)$ denote the set of elements $L \in \mathscr{L}(M)$ with $L \neq G$. Assume inductively that for every $L \in \mathscr{L}_{0}(M)$ (and for every $S$ ) that the distributions $I_{M}^{L}(\gamma)$ are defined and are supported on characters. We then define

$$
I_{M}(\gamma, f)=J_{M}(\gamma, f)-\sum_{L \in \mathscr{L}_{0}(M)} \widehat{I}_{M}^{L}\left(\gamma, \phi_{L}(f)\right) .
$$

The invariance of $I_{M}(\gamma)$ follows easily from the two formulas above. (See [1(a), Proposition 4.1].) We shall carry this induction assumption throughout the rest of this paper and also for much of the next one. The argument will be completed only by Corollary 5.3 of [1(f)], in which we shall show that $I_{M}^{G}(\gamma)$ is also supported on characters. Only then will $\widehat{I}_{M}(\gamma)$ be defined and will we be able to write

$$
J_{M}(\gamma, f)=\sum_{L \in \mathscr{L}(M)} \hat{I}_{M}^{L}\left(\gamma, \phi_{L}(f)\right) .
$$

In the paper [1(d)] we investigated the local behavior of $J_{M}(\gamma, f)$ as a function of $\gamma$. It is easy to see that $I_{M}(\gamma, f)$ has similar properties. They can all be established inductively from the corresponding properties of $J_{M}(\gamma, f)$. For example, if $\gamma$ is a general element in $M\left(F_{S}\right), J_{M}(\gamma, f)$ is given in [1(d), (6.5)] by a limit

$$
J_{M}(\gamma, f)=\lim _{a \rightarrow 1} \sum_{L \in \mathscr{L}(M)} r_{M}^{L}(\gamma, a) J_{L}(a \gamma, f) .
$$

The functions $r_{M}^{L}(\gamma, a)$ here are defined in $\S 5$ of [1(d)] in terms of a certain $(G, M)$-family, and the limit is taken over $a$ in $A_{M \text {,reg }}\left(F_{S}\right)$, the set of points in $A_{M}\left(F_{S}\right)$ whose centralizer in $G\left(F_{S}\right)$ equals $M\left(F_{S}\right)$. Assume inductively that

$$
I_{M}^{M_{1}}(\gamma, g)=\lim _{a \rightarrow 1} \sum_{L \in \mathscr{L}^{M_{1}}(M)} r_{M}^{L}(\gamma, a) I_{L}^{M_{1}}(a \gamma, g),
$$

for any $M_{1} \in \mathscr{L}_{0}(M)$ and $g \in \mathscr{I}_{\text {ac }}\left(M_{1}\left(F_{S}\right)\right)$. A similar formula then holds if $I_{M}^{M_{1}}$ is replaced by $\widehat{I}_{M}^{M_{1}}$. It follows from the definition (2.1) that $I_{M}(\gamma, f)$ equals

$$
\lim _{a \rightarrow 1} \sum_{L \in \mathscr{L}(M)} r_{M}^{L}(\gamma, a)\left(J_{L}(a \gamma, f)-\sum_{M_{1} \in \mathscr{L}_{0}(L)} \widehat{I}_{L}^{M_{1}}\left(a \gamma, \phi_{M_{1}}(f)\right)\right) .
$$

Applying the definition again, we see that

$$
I_{M}(\gamma, f)=\lim _{a \rightarrow 1} \sum_{L \in \mathscr{L}(M)} r_{M}^{L}(\gamma, a) I_{L}(a \gamma, f),
$$

with $a \in A_{M \text {,reg }}\left(F_{S}\right)$. More generally, suppose that $L_{1} \in \mathscr{L}(M)$. The induced space $\gamma^{L_{1}} \subset L_{1}\left(F_{S}\right)$ was defined in $\S 6$ of [1(d)]. It is a finite union of $L_{1}^{0}\left(F_{S}\right)$-orbits. In Corollary 6.3 of $[1(\mathrm{~d})]$ we found that

$$
J_{L_{1}}\left(\gamma^{L_{1}}, f\right)=\lim _{a \rightarrow 1} \sum_{L \in \mathscr{L}\left(L_{1}\right)} r_{L_{1}}^{L}(\gamma, a) J_{L}(a \gamma, f)
$$


with $a \in A_{M, \mathrm{reg}}\left(F_{S}\right)$. The formula

$$
I_{L_{1}}\left(\gamma^{L_{1}}, f\right)=\lim _{a \rightarrow 1} \sum_{L \in \mathscr{L}\left(L_{1}\right)} r_{L_{1}}^{L}(\gamma, a) I_{L}(a \gamma, f),
$$

with $a \in A_{M \text {,reg }}\left(F_{S}\right)$, follows inductively from this. In particular, the limit on the right exists.

Suppose that $\sigma \in M\left(F_{S}\right)$ is a semisimple element such that $G_{\sigma}$ is contained in $M$. Then

$$
J_{M}(\gamma, f) \stackrel{\left(M_{\dot{\sim}} \sigma\right)}{\sim} 0, \quad \gamma \in \sigma M_{\sigma}\left(F_{S}\right),
$$

in the notation of Lemma 2.2 of $[1(\mathrm{~d})]$. Recall that this means that $J_{M}(\gamma, f)$ coincides with the orbital integral of a smooth function of compact support on $M\left(F_{S}\right)$ for $\gamma$ near $\sigma$ in $\sigma M_{\sigma}\left(F_{S}\right)$. It follows inductively from (2.1) that the same property, namely

$$
I_{M}(\gamma, f)^{\left(M_{\dot{\sim}} \sigma\right)} 0, \quad \gamma \in \sigma M_{\sigma}\left(F_{S}\right),
$$

holds for the invariant distributions.

The distribution $I_{M}(\gamma)$ depends only on the $M^{0}\left(F_{S}\right)$-orbit of $\gamma$ since the same is true of $J_{M}(\gamma)$. More generally suppose that $y$ belongs to $M^{0}\left(F_{S}\right) G^{0}(F)$. Then $y^{-1} M y$ is another Levi subset of $G$. If $f$ belongs to $\mathscr{H}\left(G\left(F_{S}\right)\right)$, the function

$$
f^{y}(x)=f\left(y x y^{-1}\right)
$$

belongs to the Hecke space with respect to the maximal compact subgroup $y^{-1} K y$. We have the formula

$$
J_{y^{-1} M y}\left(y^{-1} \gamma y, f^{y}\right)=J_{M}(\gamma, f) .
$$

(See the remark following the proof of Lemma 8.1 of [1(d)].) It follows from (2.1) that

$$
I_{y^{-1} M y}\left(y^{-1} \gamma y, f^{y}\right)=I_{M}(\gamma, f) .
$$

Suppose that $y$ belongs to

$$
M^{0}\left(F_{S}\right) G^{0}(F) \cap K .
$$

Then it is not hard to show that

$$
I_{y^{-1} M y}\left(y^{-1} \gamma y, f\right)=I_{M}(\gamma, f)
$$

since $I_{M}(\gamma)$ is invariant.

Consider the case that $S$ consists of one non-Archimedean valuation $v$ and that $F=F_{v}=F_{S}$. Let $\sigma$ be a semisimple element in $M(F)$. In Proposition 9.1 of $[1(d)]$ we established a germ expansion

$$
J_{M}(\gamma, f) \stackrel{\left(M_{\dot{\sim}} \sigma\right)}{\sum_{L \in \mathscr{L}(M)}} \sum_{\delta \in\left(\sigma \mathscr{L}_{L_{\sigma}}(F)\right)} g_{M}^{L}(\gamma, \delta) J_{L}(\delta, f) .
$$


(See [1(d), §9] for an explanation of the notation.) It follows inductively from (2.1) that

$$
I_{M}(\gamma, f)^{(M, \dot{\sim} \sigma)} \sum_{L \in \mathscr{L}(M)} \sum_{\delta \in\left(\sigma \mathscr{L _ { L }}(F)\right)} g_{M}^{L}(\gamma, \delta) I_{L}(\delta, f) .
$$

Consider finally the case that $F=F_{v}=F_{S}$ is an Archimedean local field. Suppose that $T$ is a "maximal torus" of $G$ over $F$, in the sense of $\S 1$ of [1(d)]. If $z$ belongs to the center of the associated universal enveloping algebra, we have the differential equation

$$
J_{M}(\gamma, z f)=\sum_{L \in \mathscr{L}(M)} \partial_{M}^{L}\left(\gamma, z_{L}\right) J_{L}(\gamma, f),
$$

for $\gamma$ in the open set $T_{\text {reg }}(F)$ of $G$-regular elements in $T(F)$ [1(d), Proposition 11.1]. Using the definition (2.1) inductively again, we convert this to a differential equation

$$
I_{M}(\gamma, z f)=\sum_{L \in \mathscr{L}(M)} \partial_{M}^{L}\left(\gamma, z_{L}\right) I_{L}(\gamma, f), \quad \gamma \in T_{\mathrm{reg}}(F)
$$

for the invariant distributions. The behavior of $I_{M}(\gamma, f)$ as $\gamma$ approaches the singular set is also identical with that of $J_{M}(\gamma, f)$. In particular, the jump around a semiregular point of noncompact type can be computed for any derivative of $J_{M}(\gamma, f)$. It is given by a formula

$$
\lim _{r \rightarrow 0}\left(\partial(u) I_{M}^{\beta}\left(\gamma_{r}, f\right)-\partial(u) I_{M}^{\beta}\left(\gamma_{-r}, f\right)\right)=n_{\beta} \lim _{s \rightarrow 0}\left(\partial\left(u_{1}\right) I_{M_{1}}\left(\delta_{s}, f\right)\right),
$$

which is the analogue of Proposition 13.1 of [1(d)]. Similarly, Proposition 13.2 of $[1(d)]$ becomes

$$
\left|\partial(u) I_{M}(\gamma, f)\right| \leq c(f)\left|D^{G}(\gamma)\right|^{-q}, \quad \gamma \in \Delta_{\text {reg }} .
$$

These results follow once again inductively from the definition (2.1).

We conclude the paragraph with a lemma which will be needed for global applications.

Lemma 2.1. Suppose that $v$ is an unramified finite valuation and that $f$ is a function in $\mathscr{H}_{\mathrm{ac}}\left(G\left(F_{v}\right)\right)$ which is bi-invariant under $K_{v}$. Then

$$
I_{M}(\gamma, f)=J_{M}(\gamma, f), \quad \gamma \in M\left(F_{v}\right) .
$$

Proof. Suppose that $L \in \mathscr{L}_{0}(M)$. Then

$$
\phi_{L}(f, \pi, X)=\int_{i \mathrm{a}_{L, V}^{*}, \mathrm{i}_{G, v}^{*}} \operatorname{tr}\left(\mathscr{R}_{L}\left(\pi_{\lambda}, Q_{0}\right) \mathscr{I}_{Q_{0}}\left(\pi_{\lambda}, f^{Z}\right)\right) d \lambda,
$$

in the notation of [1(e), §7]. Here $\pi$ is a representation in $\Pi_{\text {temp }}\left(L\left(F_{v}\right)\right), X$ is a point in $\mathfrak{a}_{L, v}$ whose projection onto $\mathfrak{a}_{G, v}$ equals $Z$, and $Q_{0}$ is any element in $\mathscr{P}(L)$. Since $f$ is bi-invariant under $K_{v}$, the operator $\mathscr{I}_{Q_{0}}\left(\pi_{\lambda}, f^{Z}\right)$ vanishes unless $\pi$ is unramified. Suppose then that $\pi$ is unramified. Let $\phi$ be a vector 
in the space on which $\mathscr{I}_{Q_{0}}\left(\pi_{\lambda}\right)$ acts which is fixed by $K_{v}$. By the condition $\left(R_{8}\right)$ in [1(e), Theorem 2.1], the normalized intertwining operators

$$
R_{Q \mid Q_{0}}\left(\pi_{\lambda}\right), \quad Q \in \mathscr{P}(L),
$$

take values at $\phi$ which are independent of $\lambda$. Recalling the definitions in [1(e)], we see that

$$
\begin{aligned}
\mathscr{R}_{L}\left(\pi_{\lambda}, Q_{0}\right) \phi & =\lim _{\nu \rightarrow 0} \sum_{Q \in \mathscr{P}(L)}\left(R_{Q \mid Q_{0}}\left(\pi_{\lambda}\right)^{-1} R_{Q \mid Q_{0}}\left(\pi_{\lambda+\nu}\right) \phi\right) \theta_{Q}(\nu)^{-1} \\
& =\left(\lim _{\nu \rightarrow 0} \sum_{Q \in \mathscr{P}(L)} \theta_{Q}(\nu)^{-1}\right) \phi \\
& =0 .
\end{aligned}
$$

It thus follows that the function $\phi_{L}(f)$ vanishes. The lemma is then an immediate consequence of the definition (2.1).

\section{The invariant Distributions $I_{M}(\pi, X)$}

Next we shall define the other family of invariant distributions which occur in the trace formula. These distributions are parametrized by pairs

$$
(\pi, X), \quad \pi \in \Pi\left(M\left(F_{S}\right)\right), X \in \mathfrak{a}_{M, S} .
$$

They are related to the weighted characters

$$
J_{M}(\pi, X, f), \quad f \in \mathscr{H}\left(G\left(F_{S}\right)\right),
$$

studied in $[1(\mathrm{e})]$

In $\S 7$ of [1(e)] we observed that $J_{M}(\pi, X, f)$ was dependent only on the restriction of $f$ to $G\left(F_{S}\right)^{Z}$ for $Z=h_{G}(X)$. Thus, as with the weighted orbital integrals, the weighted characters may be regarded as distributions on $\mathscr{H}_{\text {ac }}\left(G\left(F_{S}\right)\right)$. It follows from Lemma 6.2 of [1(e)] that

$$
J_{M}\left(\pi, X, L_{h} f\right)=\sum_{Q \in \mathscr{F}(M)} J_{M}^{M_{Q}}\left(\pi, X, R_{Q, h} f\right),
$$

for any $f \in \mathscr{H}_{\text {ac }}\left(G\left(F_{S}\right)\right)$ and $h \in \mathscr{H}\left(G^{0}\left(F_{S}\right)^{1}\right)$. Since a similar formula holds for the map $\phi_{M}$, we shall define an invariant distribution

$$
I_{M}(\pi, X, f)=I_{M}^{G}(\pi, X, f), \quad f \in \mathscr{H}_{\mathrm{ac}}\left(G\left(F_{S}\right)\right),
$$

inductively by setting

$$
I_{M}(\pi, X, f)=J_{M}(\pi, X, f)-\sum_{L \in \mathscr{L}_{0}(M)} \hat{I}_{M}^{L}\left(\pi, X, \phi_{L}(f)\right) .
$$

Included in the definition is the induction assumption that for any $L \in \mathscr{L}_{0}(M)$ and any pair $(\pi, X)$ the distribution $I_{M}^{L}(\pi, X)$ is supported on characters. Observe that this induction hypothesis is our second. Before we are done, we 
shall be forced to take on several more of the same kind. All but one of these will be resolved presently. We shall show in $\S 6$ that our induction hypotheses are all contained in the one of $\S 2$. But as we have already remarked, we shall carry the hypothesis of $\S 2$ into the next paper.

Lemma 3.1. Suppose that $\pi$ is tempered. Then

$$
I_{M}(\pi, X, f)= \begin{cases}f_{G}(\pi, X), & \text { if } M=G, \\ 0, & \text { if } M \neq G .\end{cases}
$$

Proof. If $M=G$, we have $I_{G}(\pi, X, f)=f_{G}(\pi, X)$, by definition, even if $\pi$ is not tempered. If $M \neq G$, the definitions also imply that

$$
J_{M}(\pi, X, f)=\phi_{M}(f, \pi, X)=\widehat{I}_{M}^{M}\left(\pi, X, \phi_{M}(f)\right),
$$

as long as $\pi$ is tempered. The lemma follows inductively from (3.1).

At first glance, one might guess that the lemma holds for arbitrary $\pi$. However, this is decidedly not the case. If $\pi$ is not tempered and if $M \neq G$ the difference

$$
J_{M}(\pi, X, f)-\phi_{M}(f, \pi, X)
$$

is no longer 0 . For $J_{M}(\pi, X, f)$ is defined directly as an integral over $\left\{\pi_{\lambda}\right\}$, whereas $\phi_{M}(f, \pi, X)$ is defined by analytic continuation from such integrals taken over tempered representations. One finds that the difference depends in a complicated way on the residues discussed in $\S 8$ of [1(e)]. We shall say more about this in another paper.

On the other hand $I_{M}(\pi, X, f)$ does not assume too many values. Set

$$
I_{M, \mu}(\pi, X, f)=I_{M}\left(\pi_{\mu}, X, f\right) e^{-\mu(X)}, \quad \mu \in \mathfrak{a}_{M}^{*},
$$

and consider this expression as a function of $\mu$.

Lemma 3.2. (a) As a function of $\mu, I_{M, \mu}(\pi, X, f)$ is locally constant on the complement of a finite set of hyperplanes of the form $\mu\left(\alpha^{\vee}\right)=N$, for $N \in \mathbb{R}$ and $\alpha$ a root of $\left(G, A_{M}\right)$.

(b) For each $P \in \mathscr{P}(M)$, let $\varepsilon_{P}$ be a small point in the chamber $\left(\mathfrak{a}_{P}^{*}\right)^{+}$, and set $^{1}$

$$
\omega_{P}=\operatorname{vol}\left(\mathfrak{a}_{P}^{+} \cap B\right) \operatorname{vol}(B)^{-1},
$$

where $B$ is a ball in $\mathfrak{a}_{M}$ centered at the origin. Then

$$
I_{M, \mu}=\sum_{P \in \mathscr{P}(M)} \omega_{P} I_{M, \mu+\varepsilon_{P}}(\pi, X, f) .
$$

Proof. The definition (3.1) may be rewritten

$$
I_{M, \mu}(\pi, X, f)=J_{M, \mu}(\pi, X, f)-\sum_{L \in \mathscr{L}_{0}(M)} \hat{I}_{M, \mu}^{L}\left(\pi, X, \phi_{L}(f)\right),
$$

${ }^{1}$ I am indebted to the referee for pointing out that the numbers $\omega_{P}, P \in \mathscr{P}(\boldsymbol{M})$, need not be equal. 
where

$$
J_{M, \mu}(\pi, X, f)=J_{M}\left(\pi_{\mu}, X, f\right) e^{-\mu(X)} .
$$

The first assertion (a) of the lemma will follow inductively from this if we can establish the corresponding statement for $J_{M, \mu}(\pi, X, f)$. We may assume that $f$ belongs to $\mathscr{H}\left(G\left(F_{S}\right)\right)$. Then if $\mu \in \mathfrak{a}_{M}^{*}$ is in general position, we have

$$
J_{M, \mu}(\pi, X, f)=\int_{\mu+i a_{M, S}^{*}} J_{M}\left(\pi_{\lambda}, f\right) e^{-\lambda(X)} d \lambda .
$$

The required assertion then follows from the properties of the function $J_{M}\left(\pi_{\lambda}, f\right)$. (See $\S 6$ of [1(e)].) This proves (a).

Assume inductively that (b) holds if $G$ is replaced by any element $L \in$ $\mathscr{L}_{0}(M)$. Then

$$
\widehat{I}_{M, \mu}^{L}\left(\pi, X, \phi_{L}(f)\right)=\sum_{R \in \mathscr{P} L(M)} \omega_{R} \widehat{I}_{M, \mu+\varepsilon_{R}}^{L}\left(\pi, X, \phi_{L}(f)\right) .
$$

If we apply the assertion (a) to $L$, we see that this may be written as

$$
\sum_{P \in \mathscr{P}(M)} \omega_{P} \widehat{I}_{M, \mu+\varepsilon_{P}}^{L}\left(\pi, X, \phi_{L}(f)\right) .
$$

But it is an immediate consequence of the definition [1(e), §7] of $J_{M}\left(\pi_{\mu}, X, f\right)$ that

$$
J_{M, \mu}(\pi, X, f)=\sum_{P \in \mathscr{P}(M)} \omega_{P} J_{M, \mu+\varepsilon_{P}}(\pi, X, f) .
$$

The second part (b) of the lemma follows from $\left(3.1^{*}\right)$.

Remark. The reader might want to keep a special case in mind. Suppose that $F=\mathbb{R}, \pi$ is tempered, and $M=A_{M}$ (so in particular, $G$ is a connected Chevalley group). Then from the reducibility properties of the representations $\mathscr{I}_{P}\left(\pi_{\mu}\right)$, one can see that the singular hyperplanes are all of the form

$$
\mu\left(\alpha^{\vee}\right)=n, \quad \alpha \in \Sigma\left(G, A_{M}\right), n \in \mathbb{Z} .
$$

Therefore, $I_{M, \mu}(\pi, X, f)$ is constant on the affine Weyl chambers of $\mathfrak{a}_{M}^{*}$.

Lemma 3.3. Suppose that $\pi \in \Pi\left(M\left(F_{S}\right)\right)$ is unitary. Then the function

$$
I_{M, \mu}(\pi, X, f), \quad \mu \in \mathfrak{a}_{M}^{*},
$$

is constant for $\mu$ in a neighborhood of the origin.

Proof. First consider the function $J_{M, \mu}(\pi, X, f)$. As in the proof of the last lemma, we can assume that $f$ belongs to $\mathscr{H}\left(G\left(F_{S}\right)\right)$, so that

$$
J_{M, \mu}(\pi, X, f)=\int_{\mu+i \mathfrak{a}_{M . S}^{*}} J_{M}\left(\pi_{\lambda}, f\right) e^{-\lambda(X)} d \lambda .
$$

By definition [1(e), §6],

$$
J_{M}\left(\pi_{\lambda}, f\right)=\operatorname{tr}\left(\mathscr{R}_{M}\left(\pi_{\lambda}, P_{0}\right) \mathscr{I}_{P_{0}}\left(\pi_{\lambda}, f\right)\right),
$$


where $\mathscr{R}_{M}\left(\pi_{\lambda}, P_{0}\right)$ is constructed from the normalized intertwining operators

$$
R_{P \mid P_{0}}\left(\pi_{\lambda}\right): \mathscr{I}_{P_{0}}\left(\pi_{\lambda}\right) \rightarrow \mathscr{I}_{P}\left(\pi_{\lambda}\right), \quad P, P_{0} \in \mathscr{P}(M) .
$$

In particular, $J_{M}\left(\pi_{\lambda}, f\right)$ is regular at any point $\lambda$ where the intertwining operators are all regular. But by Theorem 2.1 of [1(e)], the operators $R_{P \mid P_{0}}\left(\pi_{\lambda}\right)$ are unitary whenever $\pi_{\lambda}$ is unitary. It follows that $J_{M}\left(\pi_{\lambda}, f\right)$ is regular if the real part of $\lambda$ is near 0 . By changing the contour in the integral above, we see that $J_{M, \mu}(\pi, X, f)$ is constant for $\mu$ near 0 . The lemma then follows inductively from the formula $\left(3.1^{*}\right)$.

For future reference, we state a variant of the last lemma. Its proof is similar. Lemma 3.4. Suppose that $\pi \in \Pi\left(M\left(F_{S}\right)\right)$ is unitary and that $L \in \mathscr{L}(M)$. Then

$$
I_{L}\left(\pi_{\lambda}^{L}, h_{L}(X), f\right), \quad \lambda \in \mathfrak{a}_{M, \mathbb{C}}^{*},
$$

is analytic for the real part of $\lambda$ near 0 .

It is sometimes appropriate to take a standard representation $\rho \in \Sigma\left(M\left(F_{S}\right)\right)$ $[1(\mathrm{e}), \S 5]$ instead of the irreducible $\pi$. We noted in $\S 7$ of [1(e)] that the distributions $J_{M}(\rho, X, f)$ could be defined in the same way as $J_{M}(\pi, X, f)$. We then showed [1(e), Proposition 7.1] that for any $\pi \in \Pi\left(M\left(F_{S}\right)\right), J_{M}(\pi, X, f)$ had an expansion

$$
\sum_{P} \omega_{P} \sum_{L} \sum_{\rho} \int_{\varepsilon_{P}+i a_{M, S}^{*} / \mathrm{ia}_{L, S}^{*}} r_{M}^{L}\left(\pi_{\lambda}, \rho_{\lambda}\right) J_{L}\left(\rho_{\lambda}^{L}, h_{L}(X), f\right) e^{-\lambda(X)} d \lambda,
$$

with $P, L$, and $\rho$ summed over $\mathscr{P}(M), \mathscr{L}(M)$, and $\left\{\Sigma\left(M\left(F_{S}\right)\right)\right\}$ respectively. (The notation here follows [1(e)]. In particular, $r_{M}^{L}\left(\pi_{\lambda}, \rho_{\lambda}\right)$ is a meromorphic function obtained from the ratios of the normalizing factors for $\pi_{\lambda}$ and $\rho_{\lambda}$. As in Proposition 5.1 of [1(e)], we write $\left\{\Sigma\left(M\left(F_{S}\right)\right)\right\}$ and $\left\{\Pi\left(M\left(F_{S}\right)\right)\right\}$ for the set of orbits of the finite group

$$
\Xi_{S}=\prod_{v \in S} \operatorname{Hom}\left(M^{+}\left(F_{v}\right) / M^{0}\left(F_{v}\right), \mathbb{C}^{*}\right)
$$

in $\Sigma\left(M\left(F_{S}\right)\right)$ and $\Pi\left(M\left(F_{S}\right)\right)$ respectively.) Arguing as in the proof of Lemma 3.2(b), we obtain a similar expansion

$$
I_{M}(\pi, X, f)=\sum_{P} \omega_{P} \sum_{L} \sum_{\rho} \int_{\varepsilon_{P}+i a_{M, S}^{*} / i a_{L, S}^{*}} r_{M}^{L}\left(\pi_{\lambda}, \rho_{\lambda}\right) I_{L}\left(\rho_{\lambda}^{L}, h_{L}(X), f\right) e^{-\lambda(X)} d \lambda
$$

in terms of the invariant distributions defined by the analogue of (3.1).

\section{SOME FURTHER MAPS AND DISTRIBUTIONS}

In this section we shall study some supplementary maps and distributions. These do not appear in the trace formula, but they will be needed to relate the two families of distributions we have already described. 
The function $X \rightarrow \phi_{M}(f, \pi, X)$ does not have compact support. Our first task will be to define a different map ${ }^{c} \phi_{M}$, with the property that for any $f \in \mathscr{H}\left(G\left(F_{S}\right)\right)$,

$$
X \rightarrow{ }^{c} \phi_{M}(f, \pi, X)
$$

does have compact support. However, the latter function turns out not to be smooth in $X$. In order to describe it properly, we must first introduce some larger function spaces.

Suppose that $\Phi$ is a finite set of hyperplanes in Euclidean space $\mathfrak{a}$. The complement of $\Phi$ in $\mathfrak{a}$ is a union of a finite set $\mathscr{C}$ of open connected components. If $(c, X), c \in \mathscr{C}, X \in \mathfrak{a}$, is any given pair, we set

$$
\omega(c, X)=\operatorname{vol}\left(c \cap B_{X}\right)\left(\operatorname{vol}\left(B_{X}\right)\right)^{-1},
$$

where $B_{X}$ is a small ball in $\mathfrak{a}$ centered at $X$. This function vanishes for any $X$ outside the closure of $c$. Notice also that $\sum_{c \in \mathscr{C}} \omega(c, X)=1$. As a function of $X, \omega(c, X)$ is locally constant on the strata of $\mathfrak{a}$ defined by intersections of planes in $\Phi$. Suppose that $\Phi^{\prime}$ is a subset of $\Phi$. Then any element $c^{\prime}$ in the corresponding set $\mathscr{C}^{\prime}$ of components is a union of elements in $\mathscr{C}$ together with a set of measure 0 . It is obvious that

$$
\omega\left(c^{\prime}, X\right)=\sum_{\left\{c \in \mathscr{C}: c \subseteq c^{\prime}\right\}} \omega(c, X) .
$$

We take a to be $\mathfrak{a}_{G}$. For a given set $\Phi$, we define $\mathscr{H}^{\Phi}\left(G\left(F_{S}\right)\right)$ to be the space of functions $f$ on $G\left(F_{S}\right)$ such that

$$
f(x)=\sum_{c \in \mathscr{C}} \omega\left(c, H_{G}(x)\right) f_{c}(x), \quad x \in G\left(F_{S}\right),
$$

where each function $f_{c}$ belongs to $\mathscr{H}\left(G\left(F_{S}\right)\right)$. Similarly, let $\mathscr{J}^{\Phi}\left(G\left(F_{S}\right)\right)$ be the space of functions

$$
\phi: \Pi_{\text {temp }}\left(G\left(F_{S}\right)\right) \times \mathfrak{a}_{G} \rightarrow \mathbb{C}
$$

of the form

$$
\phi(\pi, X)=\sum_{c \in \mathscr{C}} \omega(c, X) \phi_{c}(\pi, X),
$$

with $\phi_{c} \in \mathscr{J}\left(G\left(F_{S}\right)\right)$. In the manner of $\S 11$ of [1(e)], we assign topologies to the two spaces. For example, we take $\mathscr{H}^{\Phi}\left(G\left(F_{S}\right)\right)$ to be a topological direct limit

$$
\underset{\Gamma}{\lim } \underset{N}{\lim } \mathscr{H}_{N}^{\Phi}\left(G\left(F_{S}\right)\right)_{\Gamma}
$$

Here $\mathscr{H}_{N}^{\Phi}\left(G\left(F_{S}\right)\right)_{\Gamma}$ denotes the space of functions $f$ such that each $f_{c}$ belongs to the space $\mathscr{H}_{N}\left(G\left(F_{S}\right)\right)_{\Gamma}$ defined in $\S 11$ of $[1(\mathrm{e})]$. The topology on $\mathscr{H}_{N}^{\Phi}\left(G\left(F_{S}\right)\right)_{\Gamma}$ is defined by the seminorms

$$
\|f\|_{D}=\sup _{c \in \mathscr{C}} \sup _{\left\{x \in G\left(F_{S}\right): H_{G}(x) \in c\right\}}\left|D f_{c}(X)\right|
$$


with $D$ a differential operator on $G\left(F_{S \cap S_{\infty}}\right)$. (We write $S_{\infty}$ for the set of Archimedean valuations of $F$.) Now, the collection of all $\Phi$ is a partially ordered set. Define

$$
\widetilde{\mathscr{H}}\left(G\left(F_{S}\right)\right)=\underset{\Phi}{\lim } \mathscr{H}^{\Phi}\left(G\left(F_{S}\right)\right)
$$

and

$$
\widetilde{\mathscr{I}}\left(G\left(F_{S}\right)\right)=\underset{\Phi}{\lim _{\Phi}} \mathscr{I}^{\Phi}\left(G\left(F_{S}\right)\right)
$$

We point out that if $S$ contains no Archimedean valuations, $\mathfrak{a}_{G, S}$ is just a lattice in $\mathfrak{a}_{G}$, and the spaces $\widetilde{\mathscr{H}}\left(G\left(F_{S}\right)\right)$ and $\widetilde{\mathcal{F}}\left(G\left(F_{S}\right)\right)$ equal $\mathscr{H}\left(G\left(F_{S}\right)\right)$ and $\mathscr{I}\left(G\left(F_{S}\right)\right)$ respectively. In general, however, they are proper extensions.

We of course also have spaces $\widetilde{\mathscr{H}}\left(L\left(F_{S}\right)\right)$ and $\widetilde{\mathcal{F}}\left(L\left(F_{S}\right)\right)$ for each $L \in$ $\mathscr{L}(M)$. In a similar fashion, we can define extensions $\widetilde{\mathscr{H}}_{\text {ac }}\left(L\left(F_{S}\right)\right)$ and $\widetilde{\mathscr{F}_{\mathrm{ac}}}\left(L\left(F_{S}\right)\right)$ of the spaces $\mathscr{H}_{\mathrm{ac}}\left(L\left(F_{S}\right)\right)$ and $\mathscr{I}_{\mathrm{ac}}\left(L\left(F_{S}\right)\right)$.

Lemma 4.1. For $L \in \mathscr{L}(M)$, suppose that $\mathscr{H}$ is one of the spaces $\mathscr{H}^{\Phi}\left(L\left(F_{S}\right)\right)$, $\widetilde{\mathscr{H}}\left(L\left(F_{S}\right)\right)$, or $\widetilde{\mathscr{H}}_{\mathrm{ac}}\left(L\left(F_{S}\right)\right)$, and $\mathscr{I}$ is the corresponding space $\mathscr{I}^{\Phi}\left(L\left(F_{S}\right)\right)$, $\widetilde{\mathcal{F}}\left(L\left(F_{S}\right)\right.$ ), or $\widetilde{\mathcal{F}}_{\mathrm{ac}}\left(L\left(F_{S}\right)\right)$. Then $g \rightarrow g_{L}, g \in \mathscr{H}$, is a continuous, open, surjective map from $\mathscr{H}$ onto $\mathscr{I}$.

Proof. As in Proposition 1.1, the lemma follows easily from its analogue for $\mathscr{H}=\mathscr{H}\left(G\left(F_{S}\right)\right)$ and $\mathscr{F}=\mathscr{I}\left(G\left(F_{S}\right)\right)$.

In $\S 12$ of [1(e)] we defined a map

$$
f \rightarrow \phi_{M, \mu}(f), \quad f \in \mathscr{H}_{\mathrm{ac}}\left(G\left(F_{S}\right)\right),
$$

for each $\mu \in \mathfrak{a}_{M}^{*}$. We then established that $\phi_{M, \mu}$ maps $\mathscr{H}_{\text {ac }}\left(G\left(F_{S}\right)\right)$ continuously to $\mathscr{F}_{\mathrm{ac}}\left(M\left(F_{S}\right)\right)$ [1(e), Theorem 12.1]. The values of the function are defined by

$$
\phi_{M, \mu}(f, \pi, X)=J_{M, \mu}(\pi, X, f), \quad \pi \in \Pi_{\text {temp }}\left(M\left(F_{S}\right)\right), X \in \mathfrak{a}_{M, S} .
$$

The value depends only on $f^{h_{G}(X)}$, so it follows that $\phi_{M, \mu}$ can be defined for any $f \in \widetilde{\mathscr{H}}_{\text {ac }}\left(G\left(F_{S}\right)\right)$. The map sends $\widetilde{\mathscr{H}}_{\mathrm{ac}}\left(G\left(F_{S}\right)\right)$ continuously to $\widetilde{\mathcal{F}}_{\mathrm{ac}}\left(M\left(F_{S}\right)\right)$. This applies in particular to $\phi_{M}$, which is the case that $\mu=0$. It follows easily that the distributions $I_{M}(\pi, X)$ and $I_{M}(\gamma)$ can be defined on $\widetilde{\mathscr{H}}_{\mathrm{ac}}\left(G\left(F_{S}\right)\right)$.

The most familiar set of hyperplanes in $\mathfrak{a}_{M}$ is the collection

$$
\Phi=\left\{\mathfrak{a}_{L}: L \in \mathscr{L}(M), \operatorname{dim}\left(A_{M} / A_{L}\right)=1\right\} .
$$

The associated components are just the usual chambers $\left\{\mathfrak{a}_{P}^{+}: P \in \mathscr{P}(M)\right\}$. We shall write

$$
\omega_{P}(X)=\omega\left(\mathfrak{a}_{P}^{+}, X\right), \quad P \in \mathscr{P}(M),
$$

so that $\omega_{P}(0)$ is the number $\omega_{P}$ referred to in $\S 3$. For each $P \in \mathscr{P}(M)$, let $\nu_{P}$ be a point in the associated chamber $\left(\mathfrak{a}_{P}^{*}\right)^{+}$in $\mathfrak{a}_{M}^{*}$ whose distance from the 
walls is very large. The function $\phi_{M, \nu_{P}}(f, \pi, X)$ is then independent of $\nu_{P}$. We define

$$
{ }^{c} \phi_{M}(f, \pi, X)=\sum_{P \in \mathscr{P}(M)} \omega_{P}(X) \phi_{M, \nu_{P}}(f, \pi, X),
$$

for $f \in \widetilde{\mathscr{H}}_{\text {ac }}\left(G\left(F_{S}\right)\right), \pi \in \Pi_{\text {temp }}\left(M\left(F_{S}\right)\right)$ and $X \in \mathfrak{a}_{M, S}$. We have already agreed that $\phi_{M, \nu_{P}}$ maps $\widetilde{\mathscr{H}}_{\mathrm{ac}}\left(G\left(F_{S}\right)\right)$ continuously to $\widetilde{\mathcal{F}}_{\mathrm{ac}}\left(M\left(F_{S}\right)\right)$. It follows easily from the definitions that $f \rightarrow{ }^{c} \phi_{M}(f)$ is a continuous map from $\widetilde{\mathscr{H}}_{\mathrm{ac}}\left(G\left(F_{S}\right)\right)$ to $\widetilde{\mathscr{J}_{\mathrm{ac}}}\left(M\left(F_{S}\right)\right)$.

The reason for introducing ${ }^{c} \phi_{M}$ is that it maps functions of compact support to functions of compact support.

Lemma 4.2. ${ }^{c} \phi_{M}$ maps $\widetilde{\mathscr{H}}\left(G\left(F_{S}\right)\right)$ continuously to $\widetilde{\mathcal{F}}\left(M\left(F_{S}\right)\right)$.

Proof. We must show that there is a positive integer $N$, depending only on the support of $f \in \widetilde{\mathscr{H}}\left(G\left(F_{S}\right)\right)$, such that ${ }^{c} \phi_{M}(f, \pi, X)$ is supported on the ball in $\mathfrak{a}_{M, S}$ of radius $N$. Looking back at the definition of ${ }^{c} \phi_{M}$, we see that it is sufficient to show that for any $P \in \mathscr{P}(M)$, and for $X$ in the closure of $\mathfrak{a}_{P}^{+} \cap \mathfrak{a}_{M, S}, \phi_{M, \nu_{P}}(f, \pi, X)$ is supported on the ball of radius $N$. Consider the decomposition (4.2) for $f$. We can of course assume that the functions on the right-hand side of this formula are each supported on a set which depends only on the support of $f$. We may therefore assume that $f$ itself belongs to $\mathscr{H}\left(G\left(F_{S}\right)\right)$. Then

$$
\phi_{M, \nu_{P}}(f, \pi, X)=\int_{\nu_{P}+i a_{M}^{*} . S} e^{-\lambda(X)} J_{M}\left(\pi_{\lambda}, f\right) d \lambda .
$$

We need only show that as a function of $X \in \mathfrak{a}_{P}^{+}$, (4.3) is supported on a ball which depends only on the support of $f$. The proof of this fact is straightforward and is similar to an argument used in the derivation of Theorem 12.1 of $[1(\mathrm{e})]$. For we have

$$
J_{M}\left(\pi_{\lambda}, f\right)=\operatorname{tr}\left(\mathscr{R}_{M}\left(\pi_{\lambda}, P_{0}\right) \mathscr{J}_{P_{0}}\left(\pi_{\lambda}, f\right)\right),
$$

in the notation of [1(e)]. There is a standard estimate for the function $\mathscr{F}_{P_{0}}\left(\pi_{\lambda}, f\right)$. (See $[1(\mathrm{e}),(12.7)]$.) Combined with the rationality properties of $\mathscr{R}_{M}$ and the classical Paley-Wiener theorem, it yields the required assertion.

The map ${ }^{c} \phi_{M}$ behaves the same way under conjugation as $\phi_{M}$.

Lemma 4.3. If $f \in \widetilde{\mathscr{H}}_{\mathrm{ac}}\left(G\left(F_{S}\right)\right)$ and $h \in \mathscr{H}\left(G^{0}\left(F_{S}\right)^{1}\right)$,

$$
{ }^{c} \phi_{M}\left(L_{h} f\right)=\sum_{Q \in \mathscr{F}(M)}{ }^{c} \phi_{M}^{M_{Q}}\left(R_{Q, h} f\right) .
$$

Proof. According to [1(e), (12.2)],

$$
\phi_{M, \nu_{P}}\left(L_{h} f\right)=\sum_{Q \in \mathscr{F}(M)} \phi_{M, \nu_{P}}^{M_{Q}}\left(R_{Q, h} f\right),
$$


for each $P \in \mathscr{P}(M)$. Therefore

$$
{ }^{c} \phi_{M}\left(L_{h} f, \pi, X\right)=\sum_{Q \in \mathscr{F}(M)} \sum_{P \in \mathscr{P}(M)} \omega_{P}(X) \phi_{M, \nu_{P}}^{M_{Q}}\left(R_{Q, h} f, \pi, X\right) .
$$

Fix $Q \in \mathscr{F}(M)$, and set $L=M_{Q}$. If $P \in \mathscr{P}(M)$, the point $\nu_{P}$ certainly belongs to the chamber $\left(\mathfrak{a}_{L \cap P}^{*}\right)^{+}$and is far from the walls. In particular, $\phi_{M, \nu_{P}}^{M_{Q}}$ depends only on the set $L \cap P$ in $\mathscr{P}^{L}(M)$. Any $R \in \mathscr{P}^{L}(M)$ is of this form, and by (4.1),

$$
\omega_{R}(X)=\sum_{\{P \in \mathscr{P}(M): P \cap L=R\}} \omega_{P}(X) .
$$

Lemma 4.3 follows.

By Lemma 4.3, ${ }^{c} \phi_{M}(f)$ and $\phi_{M}(f)$ have the same formal behavior under commutation. We can therefore copy the construction of the distributions $I_{M}(\gamma)$ and $I_{M}(\pi, X)$, but with ${ }^{c} \phi_{M}$ playing the role of $\phi_{M}$. We obtain invariant distributions

$$
\left\{{ }^{c} I_{M}(\gamma): \gamma \in M\left(F_{S}\right)\right\}
$$

and

$$
\left\{{ }^{c} I_{M}(\pi, X):(\pi, X) \in \Pi\left(M\left(F_{S}\right)\right) \times \mathfrak{a}_{M, S}\right\}
$$

on $\widetilde{\mathscr{H}}_{\mathrm{ac}}\left(G\left(F_{S}\right)\right)$ such that

$$
{ }^{c} I_{M}(\gamma, f)=J_{M}(\gamma, f)-\sum_{L \in \mathscr{L}_{0}(M)}{ }^{c} \widehat{I}_{M}^{L}\left(\gamma,{ }^{c} \phi_{L}(f)\right)
$$

and

$$
{ }^{c} I_{M}(\pi, X, f)=J_{M}(\pi, X, f)-\sum_{L \in \mathscr{L}_{0}(M)}{ }^{c} \widehat{I}_{M}^{L}\left(\pi, X,{ }^{c} \phi_{L}(f)\right)
$$

for any $f$. Included in the definition are our third and fourth induction assumptions, namely, that for any $L \in \mathscr{L}_{0}(M)$, the distributions ${ }^{c} I_{M}^{L}(\gamma)$ and ${ }^{c} I_{M}^{L}(\pi, X)$ are supported on characters. The significance of ${ }^{c} I_{M}(\gamma)$ is in the next lemma.

Lemma 4.4. Suppose that $T$ is a "maximal torus" of $M$ over $F_{S}$ (in the sense of $\S 1$ of $[1(\mathrm{~d})])$. Then for any $f \in \widetilde{\mathscr{H}}\left(G\left(F_{S}\right)\right)$, the function

$$
\gamma \rightarrow{ }^{c} I_{M}(\gamma, f), \quad \gamma \in T\left(F_{S}\right)
$$

has compact support.

Proof. It follows from [1(d), Lemma 2.1 and definition (6.5)] that the function

$$
\gamma \rightarrow J_{M}(\gamma, f), \quad \gamma \in T\left(F_{S}\right),
$$

has compact support. Assume inductively that the lemma holds if $G$ is replaced by any $L \in \mathscr{L}_{0}(M)$. By Lemma 4.2 , the function ${ }^{c} \phi_{L}(f)$ belongs to $\widetilde{\mathscr{I}}\left(L\left(F_{S}\right)\right)$. 
Lemma 4.1 then tells us that it is the image of a function on $\widetilde{\mathscr{H}}\left(L\left(F_{S}\right)\right)$. Applying the induction assumption, we obtain the compact support of

$$
\gamma \rightarrow \widehat{I}_{M}^{L}\left(\gamma, \phi_{L}(f)\right), \quad \gamma \in T\left(F_{S}\right) .
$$

The lemma then follows from (4.4).

The distribution ${ }^{c} I_{M}(\pi, X)$ is to be regarded as a companion of $I_{M}(\pi, X)$. The two have some rather similar properties. For example, if $\pi \in \Pi\left(M\left(F_{S}\right)\right)$, $X \in \mathfrak{a}_{M, S}$ and $f \in \widetilde{\mathscr{H}}_{\mathrm{ac}}\left(G\left(F_{S}\right)\right)$ are fixed, the function

$$
{ }^{c} I_{M, \mu}(\pi, X, f)={ }^{c} I_{M}\left(\pi_{\mu}, X, f\right) e^{-\mu(X)}, \quad \mu \in \mathfrak{a}_{M}^{*},
$$

satisfies the analogue of Lemma 3.2. It is locally constant on the complement of a finite set of hyperplanes defined by roots, and it satisfies the mean value property

$$
{ }^{c} I_{M, \mu}(\pi, X, f)=\sum_{P \in \mathscr{P}(M)} \omega_{P}^{c} I_{M, \mu+\varepsilon_{P}}(\pi, X, f) .
$$

Moreover, when $\pi$ is tempered and $X$ is in general position, there is an open set on which (4.6) vanishes. However, for ${ }^{c} I_{M, \mu}$ the open set is an infinite chamber which depends on $X$.

Lemma 4.5. Suppose that $\pi$ is tempered, $f \in \widetilde{\mathscr{H}}_{\mathrm{ac}}\left(G\left(F_{S}\right)\right)$, and $M \neq G$. Then

$$
I_{M}(\pi, X, f)=0 \text {, }
$$

and

$$
\sum_{P \in \mathscr{P}(M)} \omega_{P}(X)^{c} I_{M, \nu_{P}}(\pi, X, f)=0 .
$$

Proof. Assertion (a) is just Lemma 3.1. We have included it here only for the sake of comparison.

For assertion (b), we begin by observing that

$$
\begin{aligned}
{ }^{c} \phi_{M}(f, \pi, X) & =\sum_{P \in \mathscr{P}(M)} \omega_{P}(X) \phi_{M, \nu_{P}}(f, \pi, X) \\
& =\sum_{P \in \mathscr{P}(M)} \omega_{P}(X) e^{-\nu_{P}(X)} J_{M}\left(\pi_{\nu_{P}}, X, f\right) .
\end{aligned}
$$

Therefore, the given expression,

$$
\sum_{P \in \mathscr{P}(M)} \omega_{P}(X)^{c} I_{M, \nu_{P}}(\pi, X, f),
$$

is equal to the difference between ${ }^{c} \phi_{M}(f, \pi, X)$ and

$$
\sum_{P \in \mathscr{P}(M)} \omega_{P}(X) \sum_{L \in \mathscr{L}_{0}(M)}{ }^{c} \widehat{I}_{M, \nu_{P}}^{L}\left(\pi, X,{ }^{c} \phi_{L}(f)\right)
$$


Since ${ }^{c} \widehat{I}_{M, \nu_{P}}^{L}$ depends only on the element $R=P \cap L$ in $\mathscr{P}^{L}(M)$, we can argue as in the proof of Lemma 4.3. The last expression becomes

$$
\sum_{L \in \mathscr{L}_{0}(M)} \sum_{R \in \mathscr{P} L(M)} \omega_{R}(X)^{c} \widehat{I}_{M, \nu_{R}}^{L}\left(\pi, X,{ }^{c} \phi_{L}(f)\right) .
$$

We can assume inductively that the summand corresponding to any $L \neq M$ vanishes. But the summand corresponding to $L=M$ is just equal to ${ }^{c} \phi_{M}(f, \pi, X)$. It follows that the original expression vanishes.

Corollary 4.6. Suppose that $\pi, f$, and $M$ are as in the lemma and that $X$ belongs to a chamber $\mathfrak{a}_{P}^{+}, P \in \mathscr{P}(M)$. Then

$$
{ }^{c} I_{M}\left(\pi_{\nu_{P}}, X, f\right)=0 \text {. }
$$

If we try to compare $\phi_{M}(f)$ and ${ }^{c} \phi_{M}(f)$ directly, we are lead to define invariant maps

$$
\theta_{M},{ }^{c} \theta_{M}: \widetilde{\mathscr{H}}_{\mathrm{ac}}\left(G\left(F_{S}\right)\right) \rightarrow \widetilde{\mathscr{J}}_{\mathrm{ac}}\left(M\left(F_{S}\right)\right)
$$

We define them inductively by

$$
\theta_{M}(f)={ }^{c} \phi_{M}(f)-\sum_{L \in \mathscr{L}_{0}(M)} \hat{\theta}_{M}^{L}\left(\phi_{L}(f)\right)
$$

and

$$
{ }^{c} \theta_{M}(f)=\phi_{M}(f)-\sum_{L \in \mathscr{L}_{0}(M)}{ }^{c} \hat{\theta}_{M}^{L}\left({ }^{c} \phi_{L}(f)\right),
$$

for any $f \in \widetilde{\mathscr{H}}_{\mathrm{ac}}\left(G\left(F_{S}\right)\right)$. Once again, the definition includes induction assumptions, our fifth and sixth, that for any $L \in \mathscr{L}_{0}(M)$, the maps $\theta_{M}^{L}$ and ${ }^{c} \theta_{M}^{L}$ are supported on characters.

Lemma 4.7. Suppose that $\pi \in \Pi_{\text {temp }}\left(M\left(F_{S}\right)\right)$ and $f \in \widetilde{\mathscr{H}}_{\mathrm{ac}}\left(G\left(F_{S}\right)\right)$. Then

$$
\theta_{M}(f, \pi, X)=\sum_{P \in \mathscr{P}(M)} \omega_{P}(X) I_{M, \nu_{P}}(\pi, X, f),
$$

and

$$
{ }^{c} \theta_{M}(f, \pi, X)={ }^{c} I_{M}(\pi, X, f) .
$$

Proof. According to the definition, $\theta_{M}(f, \pi, X)$ equals the difference between ${ }^{c} \phi_{M}(f, \pi, X)$ and $\sum_{L \in \mathscr{L}_{0}(M)} \hat{\theta}_{M}^{L}\left(\phi_{L}(f), \pi, X\right)$. By induction, we can assume that

$$
\hat{\theta}_{M}^{L}\left(\phi_{L}(f), \pi, X\right)=\sum_{R \in \mathscr{P} L(M)} \omega_{R}(X) \widehat{I}_{M, \nu_{R}}^{L}\left(\pi, X, \phi_{L}(f)\right),
$$

for any $L \in \mathscr{L}_{0}(M)$. The summand on the right is independent of $\nu_{R}$ as long as the point remains highly regular in $\left(\mathfrak{a}_{R}^{*}\right)^{+}$. It follows from (4.1) that

$$
\hat{\theta}_{M}^{L}\left(\phi_{L}(f), \pi, X\right)=\sum_{P \in \mathscr{P}(M)} \omega_{P}(X) \widehat{I}_{M, \nu_{P}}^{L}\left(\pi, X, \phi_{L}(f)\right) .
$$


We must subtract the sum over $L \in \mathscr{L}_{0}(M)$ of this expression from the function

$$
{ }^{c} \phi_{M}(f, \pi, X)=\sum_{P \in \mathscr{P}(M)} \omega_{P}(X) \phi_{M, \nu_{P}}(f, \pi, X) .
$$

Since

the result is just

$$
\phi_{M, \nu_{P}}(f, \pi, X)=J_{M, \nu_{P}}(\pi, X, f) \text {, }
$$

$$
\sum_{P \in \mathscr{P}(M)} \omega_{P}(X) I_{M, \nu_{P}}(\pi, X, f)
$$

The equality of this expression with $\theta_{M}(f, \pi, X)$ is the required formula (4.9).

The second formula (4.10) follows by a similar inductive argument from (4.5) and (4.8).

Lemma 4.8. Suppose that $f \in \widetilde{\mathscr{H}}_{\mathrm{ac}}\left(G\left(F_{S}\right)\right), \gamma \in M\left(F_{S}\right), \pi \in \Pi\left(M\left(F_{S}\right)\right)$, and $X \in \mathfrak{a}_{M, S}$. Then the following formulas are valid.

$$
\begin{gathered}
I_{M}(\gamma, f)={ }^{c} I_{M}(\gamma, f)+\sum_{L \in \mathscr{L}_{0}(M)}{ }^{c} \widehat{I}_{M}^{L}\left(\gamma, \theta_{L}(f)\right) . \\
{ }^{c} I_{M}(\gamma, f)=I_{M}(\gamma, f)+\sum_{L \in \mathscr{L}_{0}(M)} \widehat{I}_{M}^{L}\left(\gamma,{ }^{c} \theta_{L}(f)\right) . \\
I_{M}(\pi, X, f)={ }^{c} I_{M}(\pi, X, f)+\sum_{L \in \mathscr{L}_{0}(M)}{ }^{c} \widehat{I}_{M}^{L}\left(\pi, X, \theta_{L}(f)\right) . \\
{ }^{c} I_{M}(\pi, X, f)=I_{M}(\pi, X, f)+\sum_{L \in \mathscr{L}_{0}(M)} \hat{I}_{M}^{L}\left(\pi, X,{ }^{c} \theta_{L}(f)\right) . \\
{ }^{c} \theta_{M}(f)+\sum_{L \in \mathscr{L}_{0}(M)}{ }^{c} \hat{\theta}_{M}^{L}\left(\theta_{L}(f)\right)=\theta_{M}(f)+\sum_{L \in \mathscr{L}_{0}(M)} \hat{\theta}_{M}^{L}\left({ }^{c} \theta_{L}(f)\right) \\
= \begin{cases}M=G, \\
M \neq G .\end{cases}
\end{gathered}
$$

Remark. We should keep in mind what will eventually be proved, namely that the distributions and maps above are all supported on characters. Once we know this, we will be able to change the right-hand side of each formula to a single sum over $L \in \mathscr{L}(M)$.

Proof. We assume inductively that each formula holds when $G$ is replaced by a proper Levi subset. The formulas for $G$ are then easily established from the definitions. We shall prove only (4.11).

It follows from the definitions (2.1) and (4.4) that

$$
I_{M}(\gamma, f)-{ }^{c} I_{M}(\gamma, f)=\sum_{L \in \mathscr{L}_{0}(M)}{ }^{c} \widehat{I}_{M}^{L}\left(\gamma,{ }^{c} \phi_{L}(f)\right)-\sum_{L_{1} \in \mathscr{L}_{0}(M)} \widehat{I}_{M}^{L_{1}}\left(\gamma, \phi_{L_{1}}(f)\right) .
$$


By (4.7) the first of these sums equals

$$
\sum_{L \in \mathscr{L}_{0}(M)}{ }^{c} \widehat{I}_{M}^{L}\left(\gamma, \theta_{L}(f)\right)+\sum_{L_{1} \in \mathscr{L}_{0}(M)} \sum_{L \in \mathscr{L}^{L_{1}}(M)}{ }^{c} \widehat{I}_{M}^{L}\left(\gamma, \hat{\theta}_{L}^{L_{1}}\left(\phi_{L_{1}}(f)\right)\right) .
$$

Applying (4.11) inductively to each $L_{1} \in \mathscr{L}_{0}(M)$, we obtain

$$
\sum_{L \in \mathscr{L}^{L_{1}(M)}}{ }^{c} \widehat{I}_{M}^{L}\left(\gamma, \hat{\theta}_{L}^{L_{1}}\left(\phi_{L_{1}}(f)\right)\right)=\widehat{I}_{M}^{L_{1}}\left(\gamma, \phi_{L_{1}}(f)\right) .
$$

Formula (4.11) then follows for $G$.

\section{A CONTOUR INTEGRAL}

Formulas (4.11) and (4.12) can be taken as motivation for the introduction of the maps $\theta_{M}$ and ${ }^{c} \theta_{M}$. The two formulas describe the asymptotic behavior of $I_{M}(\gamma)$. Their value lies in the fact that they consist entirely of invariant distributions. Of course it is the compact support (Lemma 4.4) of ${ }^{c} I_{M}(\gamma)$ that is essential here. We point out that this property has come at the expense of properties of smoothness. The original distribution $I_{M}(\gamma, f)$ is not smooth in $\gamma$, but its singularities are not too bad. For example, if $F=\mathbb{R},(2.7)$ provides a simple formula for its jumps across singular hyperplanes. The singularities of ${ }^{c} \widehat{I}_{M}(\gamma, f)$ are more complicated. The same sort of thing is true of ${ }^{c} \phi_{M}$, ${ }^{c} I_{M}(\pi, M)$, and ${ }^{c} \theta_{M}$. Each of these objects has better support properties than the original one but has worse properties of smoothness.

The distributions $\left\{I_{M}^{L}(\pi, X)\right\}$ and $\left\{{ }^{c} I_{M}^{L}(\pi, X)\right\}$ and the maps $\left\{\theta_{M}^{L}\right\}$ and $\left\{{ }^{c} \theta_{M}^{L}\right\}$ are closely related. It turns out that all of these objects can be computed from each other. By (4.15), either of the two sets of maps can be computed from the other one. By Lemma 4.7, the maps can in turn be computed from either of the families of distributions. The other family of distributions could then be obtained from (4.13) and (4.14). To complete the picture, we need to establish a formula for ${ }^{c} I_{M}(\pi, X)$ in terms of the map ${ }^{c} \theta_{M}$. In this section we shall show how to write ${ }^{c} I_{M}(\pi, X)$ as a sum of contour integrals of a certain meromorphic function. This meromorphic function is derived from ${ }^{c} \theta_{M}$ in the same way that the weighted character

$$
\phi_{M}\left(f, \pi_{\lambda}\right)=J_{M}\left(\pi_{\lambda}, f\right)
$$

can be obtained from the map $\phi_{M}$. We shall review this latter construction first.

Suppose that $\pi \in \Pi_{\text {temp }}\left(M\left(F_{S}\right)\right)$, and let $\Gamma$ be a finite subset of $\Pi(K)$. For the moment, take $f$ to be a function in $\mathscr{H}\left(G\left(F_{S}\right)\right)_{\Gamma}$. The original definition of $\phi_{M}(f)$ was given in $\S 7$ of [1(e)]. Recall that

$$
\phi_{M}(f, \pi, X)=\int_{i \mathrm{a}_{M . S}^{*}} \phi_{M}\left(f, \pi_{\lambda}\right) e^{-\lambda(X)} d \lambda,
$$

where

$$
\phi_{M}\left(f, \pi_{\lambda}\right)=J_{M}\left(\pi_{\lambda}, f\right)=\operatorname{tr}\left(\mathscr{R}_{M}\left(\pi_{\lambda}, P_{0}\right) \mathscr{J}_{P_{0}}\left(\pi_{\lambda}, f\right)\right), \quad \lambda \in \mathfrak{a}_{M, \mathbb{C}}^{*},
$$


in the notation of [1(e)]. As a function of $\lambda, \phi_{M}\left(f, \pi_{\lambda}\right)$ is meromorphic. It has finitely many poles, which lie along hypersurfaces of the form

$$
q_{v, \alpha}(\lambda)-c=0, \quad c \in \mathbb{C},
$$

where $\alpha$ is a root of $\left(G, A_{M}\right)$ and $v$ is a valuation of $F$. (As in [1(e)], $q_{v, \alpha}(\lambda)$ equals $\lambda\left(\alpha^{\vee}\right)$ if $v$ is Archimedean, and equals $q_{v}^{-\lambda\left(\alpha^{\vee}\right)}$ if $v$ is a discrete valuation with residue field of order $q_{v}$.) In fact, there is a finite product

$$
q_{\pi, \Gamma}(\lambda)=\prod_{(v, \alpha)}\left(q_{v, \alpha}(\lambda)-c_{v, \alpha}\right), \quad c_{v, \alpha} \in \mathbb{C},
$$

which depends only on $\pi$ and $\Gamma$, such that the function

$$
\lambda \rightarrow q_{\pi, \Gamma}(\lambda) \phi_{M}\left(f, \pi_{\lambda}\right)
$$

belongs to the rapidly decreasing Paley-Wiener space on $\mathfrak{a}_{M}^{*}+i \mathfrak{a}_{M, S}^{*} \cdot\left(\right.$ If $\mathfrak{a}_{M, S}=$ $\mathfrak{a}_{M}$, the definition of the Paley-Wiener space is standard. Otherwise $\mathfrak{a}_{M, S}$ is a lattice and $i \mathfrak{a}_{M, S}^{*}$ is compact. In this case, the definition is similar, except that we impose no growth condition in the imaginary direction.) More generally, $\phi_{M}\left(f, \pi_{\lambda}\right)$ is meromorphic in $\pi$. In other words, if $\mathscr{M}$ is a Levi subset of $M$ over $F_{S}$, and

$$
\pi=\sigma_{\Lambda}^{M}, \quad \sigma \in \Pi_{\text {temp }}\left(\mathscr{M}\left(F_{S}\right)\right), \Lambda \in i \mathfrak{a}_{\mathscr{M}}^{*},
$$

in the notation of $\S 6$ of [1(e)], the resulting function of $\Lambda$ extends to a meromorphic function on $\mathfrak{a}_{\mathscr{M}, S}^{*}$. From the Fourier inversion formula on $\mathfrak{a}_{M, S}$ we obtain

$$
\phi_{M}\left(f, \pi_{\lambda}\right)=\int_{\mathfrak{a}_{M S}} \phi_{M}(f, \pi, X) e^{\lambda(X)} d X .
$$

Now, suppose that $f$ belongs to the larger space $\widetilde{\mathscr{H}}\left(G\left(F_{S}\right)\right)_{\Gamma}$. Then $f$ has compact support, and we can still define

$$
\mathscr{I}_{P_{0}}\left(\pi_{\lambda}, f\right)=\int_{G\left(F_{S}\right)} f(x) \mathscr{I}_{P_{0}}\left(\pi_{\lambda}, x\right) d x, \quad P_{0} \in \mathscr{P}(M),
$$

and

$$
\phi_{M}\left(f, \pi_{\lambda}\right)=J_{M}\left(\pi_{\lambda}, f\right)=\operatorname{tr}\left(\mathscr{R}_{M}\left(\pi_{\lambda}, P_{0}\right) \mathscr{J}_{P_{0}}\left(\pi_{\lambda}, f\right)\right) .
$$

Again, $\phi_{M}\left(f, \pi_{\lambda}\right)$ is meromorphic in $\pi$. In particular, it is a meromorphic function of $\lambda$. There is a function $q_{\pi, \Gamma}(\lambda)$ of the form above such that

$$
\lambda \rightarrow q_{\pi, \Gamma}(\lambda) \phi_{M}\left(f, \pi_{\lambda}\right)
$$

belongs to the slowly increasing Paley-Wiener space on $\mathfrak{a}_{M}^{*}+i \mathfrak{a}_{M, S}^{*}$.

Lemma 5.1. The function

$$
\phi_{M}(f, \pi, X), \quad X \in \mathfrak{a}_{M, S},
$$

is rapidly decreasing on $\mathfrak{a}_{M, S}$, and we have

$$
\phi_{M}\left(f, \pi_{\lambda}\right)=\int_{\mathfrak{a}_{M}, s} \phi_{M}(f, \pi, X) e^{\lambda(X)} d X
$$


Proof. By definition [1(e)],

$$
\phi_{M}(f, \pi, X)=\int_{i \mathrm{a}_{M, S}^{*} / i_{a_{G}^{*}, S}^{*}} \operatorname{tr}\left(\mathscr{R}_{M}\left(\pi_{\lambda}, P_{0}\right) \mathscr{F}_{P_{0}}\left(\pi_{\lambda}, f^{h_{G}(X)}\right)\right) e^{-\lambda(X)} d \lambda,
$$

where $h_{G}(X)=Z$ is the projection of $X$ onto $\mathfrak{a}_{G}$, and

$$
\mathscr{I}_{P_{0}}\left(\pi_{\lambda}, f^{h_{G}(X)}\right)=\int_{G\left(F_{S}\right)^{z}} f(x) \mathscr{I}_{P_{0}}\left(\pi_{\lambda}, x\right) d x .
$$

As in (4.2), we can write $f(x)$ as a finite sum

$$
\sum_{c \in \mathscr{C}} \omega\left(c, H_{G}(x)\right) f_{c}(x),
$$

where each function $f_{c}$ belongs to $\mathscr{H}\left(G\left(F_{S}\right)\right)$. Then

$$
\phi_{M}(f, \pi, X)=\sum_{c \in \mathscr{C}} \omega(c, X) \phi_{M}\left(f_{c}, \pi, X\right) .
$$

Since each function $\phi_{M}\left(f_{c}, \pi, X\right)$ is rapidly decreasing in $X$, the same is true of $\phi_{M}(f, \pi, X)$. To prove the second assertion of the lemma, note that

$$
\mathscr{I}_{P_{0}}\left(\pi_{\lambda}, f\right)=\int_{\mathfrak{a}_{G . S}} \mathscr{I}_{P_{0}}\left(\pi_{\lambda}, f^{Z}\right) d Z
$$

since $f$ has compact support. Consequently

$$
\phi_{M}\left(f, \pi_{\lambda}\right)=\int_{\mathfrak{a}_{G, S}} \operatorname{tr}\left(\mathscr{R}_{M}\left(\pi_{\lambda}, P_{0}\right) \mathscr{I}_{P_{0}}\left(\pi_{\lambda}, f^{Z}\right)\right) d Z .
$$

The required formula (5.1) then follows from the Fourier inversion formula on $i \mathfrak{a}_{M, S}^{*} / i \mathfrak{a}_{G, S}^{*}$.

We continue to assume that $f \in \widetilde{\mathscr{H}}\left(G\left(F_{S}\right)\right)$. Copying the formula (5.1), we shall define

$$
{ }^{c} \theta_{M}\left(f, \pi_{\lambda}\right)=\int_{\mathfrak{a}_{M} . S}{ }^{c} \theta_{M}(f, \pi, X) e^{\lambda(X)} d X .
$$

For the absolute convergence of the integral, we require a lemma.

Lemma 5.2. The function

$$
{ }^{c} \theta_{M}(f, \pi, X), \quad X \in \mathfrak{a}_{M, S},
$$

is rapidly decreasing on $\mathfrak{a}_{M, S}$.

Proof. Definition (4.8) is

$$
{ }^{c} \theta_{M}(f, \pi, X)=\phi_{M}(f, \pi, X)-\sum_{L \in \mathscr{L}_{0}(M)}{ }^{c} \hat{\theta}_{M}^{L}\left({ }^{c} \phi_{L}(f), \pi, X\right) .
$$

According to Lemma 4.2, each function ${ }^{c} \phi_{L}(f)$ belongs to $\widetilde{\mathcal{F}}\left(L\left(F_{S}\right)\right)$. Lemma 5.2 then follows inductively from Lemmas 4.1 and 5.1.

For future reference, we record a corollary. It is proved exactly the same way. 
Corollary 5.3. Suppose that $S \cap S_{\infty} \neq \varnothing \quad$ (so that $\mathfrak{a}_{M, S}=\mathfrak{a}_{M}$ ), and that $\Delta$ is an invariant differential operator on $\mathfrak{a}_{M}$. Then $\Delta^{c} \theta_{M}(f, \pi, X)$ is a rapidly decreasing (piecewise smooth) function of $X \in \mathfrak{a}_{M}$.

We can now take up the study of the function (5.2). Suppose that $f$ belongs to $\widetilde{\mathscr{H}}\left(G\left(F_{S}\right)\right)_{\Gamma}$. It follows inductively from $(5.3)$ and $(5.1)$ that

$$
{ }^{c} \theta_{M}\left(f, \pi_{\lambda}\right)=\phi_{M}\left(f, \pi_{\lambda}\right)-\sum_{L \in \mathscr{L}_{0}(M)}{ }^{c} \hat{\theta}_{M}^{L}\left({ }^{c} \phi_{L}(f), \pi_{\lambda}\right) .
$$

This formula in turn tells us that ${ }^{c} \theta_{M}\left(f, \pi_{\lambda}\right)$ has properties which are similar to those of $\phi_{M}\left(f, \pi_{\lambda}\right)$. In particular, ${ }^{c} \theta_{M}\left(f, \pi_{\lambda}\right)$ is analytic in $\pi$, and therefore also in $\lambda$. Moreover, there is a function $q_{\pi, \Gamma}(\lambda)$ of the form above such that

$$
\lambda \rightarrow q_{\pi, \Gamma}(\lambda)^{c} \theta_{M}\left(f, \pi_{\lambda}\right)
$$

belongs to the slowly increasing Paley-Wiener space on $\mathfrak{a}_{M}^{*}+i \mathfrak{a}_{M, S}^{*}$. Observe that the functions $\phi_{M}\left(f, \pi_{\lambda}\right)$ and ${ }^{c} \theta_{M}\left(f, \pi_{\lambda}\right)$ can be analytically continued in $\pi$. They may therefore both be defined, as meromorphic functions of $\lambda$, if $\pi$ is replaced by a standard representation $\rho \in \Sigma\left(M\left(F_{S}\right)\right)$.

Let us now take $\pi$ to be any representation in $\Pi\left(M\left(F_{S}\right)\right)$. Motivated by Lemma 6.1 of $[1(\mathrm{e})]$, we define

$$
\phi_{M}\left(f, \pi_{\lambda}\right)=\sum_{L \in \mathscr{L}(M)} \sum_{\rho \in\left\{\Sigma\left(M\left(F_{S}\right)\right)\right\}} r_{M}^{L}\left(\pi_{\lambda}, \rho_{\lambda}\right) \phi_{L}\left(f, \rho_{\lambda}\right)
$$

and

$$
{ }^{c} \theta_{M}\left(f, \pi_{\lambda}\right)=\sum_{L \in \mathscr{L}(M)} \sum_{\rho \in\left\{\Sigma\left(M\left(F_{S}\right)\right)\right\}} r_{M}^{L}\left(\pi_{\lambda}, \rho_{\lambda}\right)^{c} \theta_{L}\left(f, \rho_{\lambda}\right) .
$$

(The functions $r_{M}^{L}\left(\pi_{\lambda}, \rho_{\lambda}\right)$, we recall, were defined in $\S 5$ of [1(e)] and were shown to be rational functions of $\left.\left\{q_{v, \alpha}(\lambda)\right)\right\}$.) Then we have

$$
{ }^{c} \theta_{M}\left(f, \pi_{\lambda}\right)=\phi_{M}\left(f, \pi_{\lambda}\right)-\sum_{L \in \mathscr{L}_{0}(M)}{ }^{c} \hat{\theta}_{M}^{L}\left({ }^{c} \phi_{L}(f), \pi_{\lambda}\right) .
$$

Once more, $\phi_{M}\left(f, \pi_{\lambda}\right)$ and ${ }^{c} \theta_{M}\left(f, \pi_{\lambda}\right)$ are meromorphic in $\lambda$. Again, there is a function $q_{\pi, \Gamma}(\lambda)$ of the form above whose product with either of them belongs to the slowly increasing Paley-Wiener space on $\mathfrak{a}_{M}^{*}+i \mathfrak{a}_{M, S}^{*}$.

Proposition 5.4. Suppose that $\pi \in \Pi\left(M\left(F_{S}\right)\right)$ and $f \in \widetilde{\mathscr{C}}\left(G\left(F_{S}\right)\right)$. Then

$$
{ }^{c} I_{M}(\pi, X, f)=\lim _{\beta} \sum_{P \in \mathscr{P}(M)} \omega_{P} \int_{\varepsilon_{P}+i a_{M}^{*} . S} \hat{\beta}(\lambda)^{c} \theta_{M}\left(f, \pi_{\lambda}\right) e^{-\lambda(X)} d \lambda,
$$

where $X$ lies in the complement of a finite set of hyperplanes and $\beta$ is a test function in $C_{c}^{\infty}\left(\mathfrak{a}_{M, S}\right)$ which approaches the Dirac measure at the origin.

Remarks. (1) The function $\hat{\beta}$ belongs to the rapidly decreasing Paley-Wiener space on $\mathfrak{a}_{M}^{*}+i \mathfrak{a}_{M, S}^{*}$, so the existence of the integrals over $\varepsilon_{P}+i \mathfrak{a}_{M, S}^{*}$ follows from the remarks above. 
(2) If none of the poles of ${ }^{c} \theta_{M}\left(f, \pi_{\lambda}\right)$ meet $i \mathfrak{a}_{M, S}^{*}$, the right-hand side of the formula simplifies to

$$
\lim _{\beta} \int_{i \mathrm{a}_{M . S}^{*}} \hat{\beta}(\lambda)^{c} \theta_{M}\left(f, \pi_{\lambda}\right) e^{-\lambda(X)} d \lambda .
$$

(3) Suppose that $S$ consists of one discrete valuation. Then $\mathfrak{a}_{M, S}$ is a lattice in $\mathfrak{a}_{M}$, and $\beta$ may be taken to be the Dirac measure. It can be removed from the formula. The formula in this case holds for all values of $X$.

Proof. We shall actually show that

$\int_{\mathfrak{a}_{M} . S} \beta(Y)^{c} I_{M}(\pi, X-Y, f) d Y=\sum_{P \in \mathscr{P}(M)} \omega_{P} \int_{\varepsilon_{P}+i a_{M}^{*}, S} \hat{\beta}(\lambda)^{c} \theta_{M}\left(f, \pi_{\lambda}\right) e^{-\lambda(X)} d \lambda$,

for any $X \in \mathfrak{a}_{M, S}$ and $\beta \in C_{c}^{\infty}\left(\mathfrak{a}_{M, S}\right)$. It follows easily from (4.5) and Lemma 4.2 that ${ }^{c} I_{M}(\pi, X, f)$ is a piecewise smooth function of $X$, whose singularities lie along a finite set of hyperplanes. The required formula of the lemma would then hold for $X$ in the complement of these hyperplanes.

We shall first derive an analogue of $(5.7)$ for $J_{M}(\pi, X, f)$. Since $f$ has compact support, the function

$$
J_{M}\left(\pi_{\lambda}, f\right)=\operatorname{tr}\left(\mathscr{R}_{M}\left(\pi_{\lambda}, P_{0}\right) \mathscr{I}_{P_{0}}\left(\pi_{\lambda}, f\right)\right)
$$

exists. It in fact equals the function $\phi_{M}\left(f, \pi_{\lambda}\right)$ introduced above. This is just the definition if $\pi$ is tempered, and the general case follows from analytic continuation, Proposition 6.1 of [1(e)], and formula (5.4). Consequently

$$
\phi_{M}\left(f, \pi_{\lambda}\right)=\int_{\mathfrak{a}_{G, S}} J_{M}\left(\pi_{\lambda}, f^{Z}\right) d Z,
$$

where

By definition,

$$
J_{M}\left(\pi_{\lambda}, f^{Z}\right)=\operatorname{tr}\left(\mathscr{R}_{M}\left(\pi_{\lambda}, P_{0}\right) \mathscr{I}_{P_{0}}\left(\pi_{\lambda}, f^{Z}\right)\right)
$$

$$
J_{M}(\pi, X, f)=\sum_{P \in \mathscr{P}(M)} \omega_{P} \int_{\varepsilon_{P}+i a_{M . S}^{*} / a_{G, S}^{*}} J_{M}\left(\pi_{\lambda}, f^{h_{G}(X)}\right) e^{-\lambda(X)} d \lambda .
$$

Combined with the Fourier inversion formula in $i \mathrm{a}_{M, S}{ }^{*}$, these facts lead without difficulty to the formula

$$
\int_{\mathfrak{a}_{M . S}} \beta(Y) J_{M}(\pi, X-Y, f) d Y=\sum_{P \in \mathscr{P}(M)} \omega_{P} \int_{\varepsilon_{P}+i \mathrm{a}_{M}^{*}, S} \hat{\beta}(\lambda) \phi_{M}\left(f, \pi_{\lambda}\right) e^{-\lambda(X)} d \lambda .
$$

We shall prove (5.7). According to (4.5), the left-hand side of (5.7) equals the difference between the left-hand side of (5.8) and

$$
\sum_{L \in \mathscr{L}_{0}(M)} \int_{\mathfrak{a}_{M . S}} \beta(Y)^{c} \widehat{I}_{M}^{L}\left(\pi, X-Y,{ }^{c} \phi_{L}(f) d Y .\right.
$$


Assume inductively that (5.7) holds for $L$. Then the last expression can be written as

$$
\sum_{L \in \mathscr{L}_{0}(M)} \sum_{R \in \mathscr{P} L(M)} \omega_{R} \int_{\varepsilon_{R}+i a_{M}^{*}, S} \hat{\beta}(\lambda)^{c} \hat{\theta}_{M}^{L}\left({ }^{c} \phi_{L}(f), \pi_{\lambda}\right) e^{-\lambda(X)} d \lambda
$$

Since ${ }^{c} \phi_{L}(f)$ belongs to $\widetilde{\mathcal{F}}\left(L\left(F_{S}\right)\right)$, the function

$$
\zeta \rightarrow{ }^{c} \hat{\theta}_{M}^{L}\left({ }^{c} \phi_{L}(f), \pi_{\lambda+\zeta}\right), \quad \zeta \in \mathfrak{a}_{L, \mathbb{C}}^{*},
$$

is entire. We can therefore translate the contour of integration by any vector in $\mathfrak{a}_{L}^{*}$. The expression may consequently be written as

$$
\sum_{L \in \mathscr{L}_{0}(M)} \sum_{P \in \mathscr{P}(M)} \omega_{P} \int_{\varepsilon_{P}+i a_{M . S}^{*}} \hat{\beta}(\lambda)^{c} \hat{\theta}_{M}^{L}\left({ }^{c} \phi_{L}(f), \pi_{\lambda}\right) e^{-\lambda(X)} d \lambda .
$$

In particular, the sum over $L$ can be taken inside the integral over $\lambda$. Thus, the left-hand side of (5.7) equals

$$
\sum_{P} \omega_{P} \int_{\varepsilon_{P}+i a_{M}^{*}, S} \hat{\beta}(\lambda)\left(\phi_{M}\left(f, \pi_{\lambda}\right)-\sum_{L \in \mathscr{L}_{0}(M)}{ }^{c} \hat{\theta}_{M}^{L}\left({ }^{c} \phi_{L}(f), \pi_{\lambda}\right)\right) e^{-\lambda(X)} d \lambda .
$$

By (5.6), this is just the required right-hand side of (5.7). The proposition is proved.

Let $\beta_{1}$ be a function in $C_{c}^{\infty}\left(\mathfrak{a}_{M, S}\right)$ which is symmetric about the origin, and set

$$
\beta_{\varepsilon}(Y)=\varepsilon^{-\operatorname{dim} \mathfrak{a}_{M}} \beta_{1}\left(\varepsilon^{-1} Y\right), \quad \varepsilon>0, Y \in \mathfrak{a}_{M, S}
$$

It is not hard to show from our definitions that

$$
{ }^{c} I_{M}(\pi, X, f)=\lim _{\varepsilon \rightarrow 0} \int_{\mathfrak{a}_{M} S} \beta_{\varepsilon}(Y)^{c} I_{M}(\pi, X-Y, f) d Y,
$$

for any $X \in \mathfrak{a}_{M, S}$. It follows from (5.8) that

$$
{ }^{c} I_{M}(\pi, X, f)=\lim _{\varepsilon \rightarrow 0} \sum_{P} \omega_{P} \int_{\varepsilon_{P}+i a_{M}^{*}, S}\left(\hat{\beta}_{\varepsilon}\right)(\lambda)^{c} \theta_{M}\left(f, \pi_{\lambda}\right) e^{-\lambda(X)} d \lambda,
$$

for any $X$. In particular, we can determine ${ }^{c} I_{M}(\pi, X, f)$ from ${ }^{c} \theta_{M}$ for all values of $X$.

\section{REDUCTION OF INDUCTION HYPOTHESES}

The distributions $I_{M}(\gamma)$ do not have compact support in $\gamma$. This circumstance is behind the existence of the distributions $I_{M}(\pi, X)$. It is also the reason we have defined the supplementary distributions and the maps $\theta_{M}$ and ${ }^{c} \theta_{M}$. The implication is that these objects could all be computed from an adequate knowledge of the asymptotic behavior of $I_{M}(\gamma)$. This will be the role of the integral formula in Proposition 5.4. The formula is actually more suited to comparing distributions on different groups than to evaluating them on a single 
group. The same is of course true of the trace formula itself. However, we can give one illustration here of how the integral formula may be applied. We shall show that ${ }^{c} I_{M}(\gamma), I_{M}(\pi, X),{ }^{c} I_{M}(\pi, X), \theta_{M}$, and ${ }^{c} \theta_{M}$ are all supported on characters, provided that the same is true of $I_{M}(\gamma)$. In other words, we shall show that induction hypotheses of $\S \S 3$ and 4 may be subsumed in those of $\S 2$.

Theorem 6.1. Fix a Levi subset $M$ and a function $f \in \widetilde{\mathscr{H}}_{\mathrm{ac}}\left(G\left(F_{S}\right)\right)$ such that $f_{G}=0$. Assume that $I_{L}(\delta, f)=0$ for each $L \in \mathscr{L}(M)$ and $\delta \in L\left(F_{S}\right)$. Then

$$
\begin{gathered}
{ }^{c} I_{M}(\gamma, f)=0, \quad \gamma \in M\left(F_{S}\right), \\
\theta_{M}(f)={ }^{c} \theta_{M}(f)=0,
\end{gathered}
$$

and

(c) $\quad I_{M}(\pi, X, f)={ }^{c} I_{M}(\pi, X, f)=0, \quad \pi \in \Pi\left(M\left(F_{S}\right)\right), X \in \mathfrak{a}_{M, S}$.

In particular, the induction hypotheses of $\S \S 3$ and 4 are all implied by original induction assumption of $\S 2$.

Proof. If $M=G$, the definitions imply that

$$
\begin{gathered}
I_{M}(\pi, X, f)={ }^{c} I_{M}(\pi, X, f)=f_{G}(\pi, X)=0, \\
{ }^{c} I_{M}(\gamma, f)=I_{M}(\gamma, f)=0,
\end{gathered}
$$

and

$$
\theta_{M}(f)={ }^{c} \theta_{M}(f)=f_{G}=0 .
$$

We may therefore assume that $M \neq G$. We may also take $f$ to be a function in $\mathscr{H}\left(G\left(F_{S}\right)\right)$. For if $Z$ equals either $h_{G}(X)$ or $H_{G}(\gamma)$, the restriction of any given function in $\widetilde{\mathscr{H}}\left(G\left(F_{S}\right)\right)$ to the set $G\left(F_{S}\right)^{Z}$ coincides with that of some function in $\mathscr{H}\left(G\left(F_{S}\right)\right)$.

Assume inductively that the theorem has been proved if $M$ is replaced by any $L \in \mathscr{L}(M)$ with $L \neq M$. By (4.12) we have

$$
{ }^{c} I_{M}(\gamma, f)=I_{M}(\gamma, f)+\sum_{L \in \mathscr{L}_{0}(M)} \hat{I}_{M}^{L}\left(\gamma,{ }^{c} \theta_{L}(f)\right), \quad \gamma \in M\left(F_{S}\right) .
$$

Our latest induction assumption then implies that ${ }^{c} \theta_{L}(f)=0$ if $L \neq M$. Combining this with the hypothesis of the theorem, we obtain

$$
{ }^{c} I_{M}(\gamma, f)=\widehat{I}_{M}^{M}\left(\gamma,{ }^{c} \theta_{M}(f)\right) .
$$

Since $f$ belongs to $\mathscr{H}\left(G\left(F_{S}\right)\right)$, Lemma 4.4 tells us that the left-hand side has bounded support as a function of $\gamma$ in the space of $M^{0}\left(F_{S}\right)$-orbits in $M\left(F_{S}\right)$. The same is therefore true of the right-hand side. For a given $X \in \mathfrak{a}_{M, S}$, the right-hand side is the orbital integral in

$$
\left\{\gamma \in M\left(F_{S}\right): H_{M}(\gamma)=X\right\}
$$


of a function defined on $M\left(F_{S}\right)^{X}$. The tempered characters of this function are just

$$
{ }^{c} \theta_{M}(f, \pi, X), \quad \pi \in \Pi_{\text {temp }}\left(M\left(F_{S}\right)\right) .
$$

Therefore, this last expression is compactly supported in $X \in \mathfrak{a}_{M, S}$. It follows that

$$
{ }^{c} \theta_{M}\left(f, \pi_{\lambda}\right)=\int_{\mathfrak{a}_{M} S}{ }^{c} \theta_{M}(f, \pi, X) e^{\lambda(X)} d \lambda
$$

is an entire function of $\lambda \in \mathfrak{a}_{M, \mathbb{C}}^{*}$.

Take a representation $\pi \in \Pi_{\text {temp }}\left(M\left(F_{S}\right)\right)$ and a point $\mu \in \mathfrak{a}_{M, S}^{*}$ in general position. Apply Proposition 5.4 to the representation $\pi_{\mu}$. We obtain

$$
\begin{aligned}
{ }^{c} \widehat{I}_{M}\left(\pi_{\mu}, X, f\right) & =\lim _{\beta} \int_{i \mathrm{a}_{M}^{*}, S} \hat{\beta}(\lambda)^{c} \theta_{M}\left(f, \pi_{\mu+\lambda}\right) e^{-\lambda(X)} d \lambda \\
& =e^{\mu(X)} \lim _{\beta} \int_{\mu+i \mathrm{a}_{M, S}^{*}} \hat{\beta}(\lambda-\mu)^{c} \theta_{M}\left(f, \pi_{\lambda}\right) e^{-\lambda(X)} d \lambda .
\end{aligned}
$$

Remember that $\beta$ is allowed to be any test function which approaches the Dirac measure at the origin. But $\lambda \rightarrow \hat{\beta}(\lambda-\mu)$ is the Fourier-Laplace transform of a function $X \rightarrow e^{-\mu(X)} \beta(X)$ which also approaches the Dirac measure at the origin. We may therefore replace $\hat{\beta}(\lambda-\mu)$ by $\hat{\beta}(\lambda)$. We obtain

$$
e^{-\mu(X) c} I_{M}\left(\pi_{\mu}, X, f\right)=\lim _{\beta} \int_{\mu+i a_{M}^{*} s} \hat{\beta}(\lambda)^{c} \theta_{M}\left(f, \pi_{\lambda}\right) e^{-\lambda(X)} d \lambda .
$$

Now, the integrand on the right is entire in $\lambda$. It follows that the integral over $\mu+i \mathfrak{a}_{M, S}^{*}$ can be deformed to any other translate of $i \mathfrak{a}_{M, S}{ }^{*}$. The outcome is that the function

$$
e^{-\mu(X) c} I_{M}\left(\pi_{\mu}, X, f\right)={ }^{c} I_{M, \mu}(\pi, X, f)
$$

is independent of $\mu$. At least this is true for almost all $\mu$ and $X$. But by the formulas in $\S 4$, the value of this function at any $\mu$ and $X$ can be expressed in terms of its values at nearby points in general position. It follows that the function is independent of $\mu$ without exception. Deforming $\mu$ to each of the points $\nu_{P}, P \in \mathscr{P}(M)$, we obtain

$$
{ }^{c} I_{M, \mu}(\pi, X, f)=\sum_{P \in \mathscr{P}(M)} \omega_{P}(X)^{c} I_{M, \nu_{P}}(\pi, X, f) .
$$

It thus follows from Lemma 4.5 that

$$
{ }^{c} I_{M, \mu}(\pi, X, f)=0 .
$$

Set $\mu=0$, and combine the last formula with that of Lemma 4.7. The result is

$$
{ }^{c} \theta_{M}(f, \pi, X)={ }^{c} I_{M}(\pi, X, f)={ }^{c} I_{M, 0}(\pi, X, f)=0,
$$

for any $\pi \in \Pi_{\text {temp }}\left(M\left(F_{S}\right)\right)$ and $X \in \mathfrak{a}_{M, S}$. Therefore, the function ${ }^{c} \theta_{M}(f)$ vanishes. The assertions of the theorem can now be easily proved. The required 
formula (a) follows immediately from (6.1). Formula (b) follows from (4.15) and the fact that the functions ${ }^{c} \theta_{L}(f), L \in \mathscr{L}(M)$, all vanish. To establish (c), fix an arbitrary representation $\pi$ in $\Pi\left(M\left(F_{S}\right)\right)$ and consider the function ${ }^{c} \theta_{M}\left(f, \pi_{\lambda}\right)$. The vanishing of ${ }^{c} \theta_{M}(f)$ means that the function is zero if $\pi$ is tempered. By analytic continuation from the tempered case, it follows that ${ }^{c} \theta_{M}\left(f, \rho_{\lambda}\right)=0$ for any standard representation $\rho \in \Sigma\left(M\left(F_{S}\right)\right)$. A similar formula is of course valid if $M$ is replaced by any element $L \in \mathscr{L}(M)$. Consequently, the expansion (5.5) implies that ${ }^{c} \theta_{M}\left(f, \pi_{\lambda}\right)=0$ in general. Apply Proposition 5.4. The formula

$$
{ }^{c} I_{M}(\pi, X, f)=0, \quad X \in \mathfrak{a}_{M, S},
$$

follows. But with what has already been proved, the formula (4.14) simplifies to

$$
I_{M}(\pi, X, f)={ }^{c} I_{M}(\pi, X, f) .
$$

This gives the final assertion (c).

\section{A PROPERTY OF $(G, M)$-FAMILIES}

We would like to investigate the descent and splitting properties of our distributions. We shall establish splitting formulas in $\S 9$. They reduce questions about the distributions to the case that $S$ contains one valuation. The descent formulas, which we shall prove in $\S 8$, reduce such questions further to the case that the data which parametrize the distributions are elliptic. Both properties were studied in the earlier paper [1(a), $\S 10,11]$ but under quite limited circumstances. Only the distributions $I_{M}(\gamma)$ were discussed there and only for $\gamma$ regular. Moreover, we need to generalize the formulas in another sense. For example, it is important to be able to rewrite the distributions in which $M$ is given over a global field, in terms of distributions indexed by Levi sets defined over local fields. We must introduce new methods. In this paragraph, we shall discuss a general descent formula for $(G, M)$-families. The formula, whose verification we will postpone until the appendix, will make the behavior of our distributions appear more transparent. In particular, it will provide a simple interpretation of the coefficients that appear in the expansions of the distributions.

Suppose that

$$
c_{P}(\lambda), \quad P \in \mathscr{P}(M), \quad \lambda \in i \mathfrak{a}_{M}^{*},
$$

is a $(G, M)$-family $([1(\mathrm{a}), \S 6],[1(\mathrm{~d}), \S 1])$. Then

$$
c_{M}(\lambda)=\sum_{P \in \mathscr{P}(M)} c_{P}(\lambda) \theta_{P}(\lambda)^{-1}, \quad \lambda \in i \mathfrak{a}_{M}^{*},
$$

is a smooth function whose value at $\lambda=0$ we generally denote $c_{M}$ [1(a), Lemma 6.3]. Recall that for each $L \in \mathscr{L}(M)$ there is an associated $(G, L)$-family

$$
c_{Q}(\lambda), \quad Q \in \mathscr{P}(L), \lambda \in i \mathfrak{a}_{L}^{*},
$$


and for every $Q \in \mathscr{F}(M)$ there is an associated $\left(M_{Q}, M\right)$-family

$$
c_{R}(\lambda), \quad R \in \mathscr{P}^{M_{Q}}(M), \lambda \in i \mathfrak{a}_{M}^{*} .
$$

For each of these we have the corresponding functions $c_{L}(\lambda)$ and $c_{M}^{Q}(\lambda)$. We shall find a formula for $c_{L}(\lambda)$ in terms of the functions $c_{M}^{Q}(\lambda)$.

We shall actually study a family of functions derived from $\left\{c_{P}(\lambda)\right\}$ which is larger than the collection $\left\{c_{L}(\lambda): L \in \mathscr{L}(M)\right\}$. This comes from a class of subspaces of $\mathfrak{a}_{M}$ which was introduced in [7, §2]. Suppose first that $\mathfrak{b}$ is any vector subspace of $\mathfrak{a}_{M}$ which contains $\mathfrak{a}_{G}$. Then

$$
\mathfrak{a}_{M}=\mathfrak{a}_{M}^{\mathfrak{b}} \oplus \mathfrak{b}^{G} \oplus \mathfrak{a}_{G},
$$

where $\mathfrak{a}_{M}^{\mathfrak{b}}$ and $\mathfrak{b}^{G}$ stand for the respective orthogonal complements of $\mathfrak{b}$ and $\mathfrak{a}_{G}$ in $\mathfrak{a}_{M}$ and $\mathfrak{b}$. By a root $\beta$ of $\mathfrak{b}$, we mean the restriction to $\mathfrak{b}$ of a root of $\left(G, A_{M}\right)$. For any such $\beta$, let $\Sigma(\beta)$ be the set of roots of $\left(G, A_{M}\right)$ whose restriction to $\mathfrak{b}$ equals $\beta$. We say that $\mathfrak{b}$ is special if for every such $\beta$, the linear function $\sum_{\alpha \in \Sigma(\beta)}\left(m_{\alpha} \alpha\right)$, in which $m_{\alpha}$ stands for the multiplicity of $\alpha$, vanishes on $\mathfrak{a}_{M}^{\mathfrak{b}}$. Assume that this is the case. The roots partition $\mathfrak{b}$ into a finite set of chambers, and to each of these corresponds a system of positive roots. We shall write $\mathscr{P}(\mathfrak{b})$ for the collection of such systems of positive roots, and we shall write

$$
\mathfrak{b}_{\mathfrak{p}}^{+}, \quad \mathfrak{p} \in \mathscr{P}(\mathfrak{b}),
$$

for the corresponding chambers in $\mathfrak{b}$. According to Lemma 2.2 of [7], every positive system $\mathfrak{p}$ in $\mathscr{P}(\mathfrak{b})$ has a uniquely determined subset $\Delta_{\mathfrak{p}}$ which has the usual properties of simple roots. Namely, $\Delta_{\mathfrak{p}}$ is linearly independent, and every element in $\mathfrak{p}$ can be represented as a nonnegative integral combination of roots in $\Delta_{\mathfrak{p}}$. Suppose that $\mathfrak{p} \in \mathscr{P}(\mathfrak{b})$. Then there is a unique element $Q \in \mathscr{F}(M)$ such that the chamber $\mathfrak{b}_{\mathfrak{p}}^{+}$is contained in $\mathfrak{a}_{Q}^{+}$. The restriction to $\mathfrak{b}$ of any root of $\left(Q, A_{M_{Q}}\right)$ belongs to $\mathfrak{p}$. It follows easily that $\Delta_{\mathfrak{p}}$ is the restriction to $\mathfrak{b}$ of a subset of the simple roots $\Delta_{Q}$.

Many of the constructions for the space $\mathfrak{a}_{M}$ can be carried over to $\mathfrak{b}$. For example, if $\mathfrak{p} \in \mathscr{P}(\mathfrak{b})$, one can define "co-roots" $\Delta_{\mathfrak{p}}^{\vee}=\left\{\alpha^{\vee}: \alpha \in \Delta_{\mathfrak{p}}\right\}$, and one can then set

$$
\theta_{\mathfrak{p}}(\lambda)=\left(\operatorname{vol}\left(\mathfrak{b}^{G} / \mathbb{Z}\left(\Delta_{\mathfrak{p}}^{\vee}\right)\right)\right)^{-1} \prod_{\alpha \in \Delta_{\mathfrak{p}}} \lambda\left(\alpha^{\vee}\right), \quad \lambda \in i \mathfrak{b}^{*} .
$$

One can also introduce the notion of an $\left(\mathfrak{a}_{G}, \mathfrak{b}\right)$-family of functions

$$
c_{\mathfrak{p}}(\lambda), \quad \mathfrak{p} \in \mathscr{P}(\mathfrak{b}), \lambda \in i \mathfrak{b}^{*},
$$

by copying the definition of a $(G, M)$-family. For any such family, the number

$$
c_{\mathfrak{b}}=\lim _{\lambda \rightarrow 0} c_{\mathfrak{b}}(\lambda)=\lim _{\lambda \rightarrow 0} \sum_{\mathfrak{p} \in \mathscr{P}(\mathfrak{b})} c_{\mathfrak{p}}(\lambda) \theta_{\mathfrak{p}}(\lambda)^{-1}
$$


is defined. Pursuing the analogy further, we let $\mathscr{L}(\mathfrak{b})$ denote the finite collection of subspaces of $\mathfrak{b}$ of the form

$$
\mathfrak{b}_{1}=\left\{H \in \mathfrak{b}: \beta_{1}(H)=\cdots=\beta_{l}(H)=0\right\},
$$

for roots $\beta_{1}, \ldots, \beta_{l}$ of $\mathfrak{b}$. Any such $\mathfrak{b}_{1}$ is also a special subspace of $\mathfrak{a}_{M}$. We write $\mathscr{F}(\mathfrak{b})$ for the set of positive systems $\mathfrak{q} \in \mathscr{P}\left(\mathfrak{b}_{1}\right)$, where $\mathfrak{b}_{1}=\mathfrak{b}_{\mathfrak{q}}$ ranges over the spaces in $\mathscr{L}(\mathfrak{b})$. For any $\left(\mathfrak{a}_{G}, \mathfrak{b}\right)$-family, and elements $\mathfrak{b}_{1} \in \mathscr{L}(\mathfrak{b})$ and $\mathfrak{q} \in \mathscr{F}(\mathfrak{b})$, there is associated an $\left(\mathfrak{a}_{G}, \mathfrak{b}_{1}\right)$-family and $\mathfrak{a}\left(\mathfrak{b}_{\mathfrak{q}}, \mathfrak{b}\right)$-family.

Suppose that $\left\{c_{P}(\lambda): P \in \mathscr{P}(M)\right\}$ is a $(G, M)$-family. If $\mathfrak{b}$ is a special subspace of $\mathfrak{a}_{M}$, we shall write $M_{\mathfrak{b}}$ for the maximal element in $\mathscr{L}(M)$ such that $\mathfrak{a}_{M_{\mathfrak{b}}}$ contains $\mathfrak{b}$. Then $M_{\mathfrak{b}}$ is the Levi subset defined by the roots of $\left(G, A_{M}\right)$ which vanish on $\mathfrak{b}$. Consider the associated $\left(G, M_{\mathfrak{b}}\right)$-family

$$
\left\{c_{Q}(\nu): Q \in \mathscr{P}\left(M_{\mathfrak{b}}\right), \nu \in i \mathfrak{a}_{M_{b}}^{*}\right\} \text {. }
$$

For any $\mathfrak{p} \in \mathscr{P}(\mathfrak{b})$, there is a unique element $Q \in \mathscr{P}\left(M_{\mathfrak{b}}\right)$ such that $\mathfrak{b}_{\mathfrak{p}}^{+}$is contained in $\mathfrak{a}_{Q}^{+}$. Define $c_{\mathfrak{p}}(\nu)=c_{Q}(\nu)$, for $\nu$ restricted to the subspace $i \mathfrak{b}^{*}$ of $i \mathfrak{a}_{M_{\mathfrak{b}}}^{*}$. Then

is an $\left(\mathfrak{a}_{G}, \mathfrak{b}\right)$-family.

$$
c_{\mathfrak{p}}(\nu), \quad \mathfrak{p} \in \mathscr{P}(\mathfrak{b}), \nu \in i \mathfrak{b}^{*},
$$

Our main result will be an expansion for $c_{\mathfrak{b}}$ in terms of $\left\{c_{M}^{Q}: Q \in \mathscr{F}(M)\right\}$. The coefficients will be certain constants

$$
d_{M}^{G}(\mathfrak{b}, L), \quad L \in \mathscr{L}(M),
$$

which we define as follows. For a given element $L \in \mathscr{L}(M)$, consider the natural map

$$
\mathfrak{a}_{M}^{\mathfrak{b}} \oplus \mathfrak{a}_{M}^{L} \rightarrow \mathfrak{a}_{M}^{G} .
$$

If the map is not an isomorphism, $d_{M}^{G}(\mathfrak{b}, L)$ is defined to be 0 . If the map is an isomorphism, we set $d_{M}^{G}(\mathfrak{b}, L)$ equal to the volume in $\mathfrak{a}_{M}^{G}$ of the parallelogram generated by orthonormal bases of $\mathfrak{a}_{M}^{\mathfrak{b}}$ and $\mathfrak{a}_{M}^{L}$. Notice that in this case, the natural map from $\mathfrak{a}_{M}^{L}$ to $\mathfrak{b}^{G}$ is also an isomorphism. If $\Gamma$ is any bounded measurable subset of $\mathfrak{a}_{M}^{L}$ and $\widetilde{\Gamma}$ is its image in $\mathfrak{b}^{G}$, then

$$
\operatorname{vol}(\widetilde{\Gamma})=d_{M}^{G}(\mathfrak{b}, L) \operatorname{vol}(\Gamma) .
$$

In the special case that $\mathfrak{b}=\mathfrak{a}_{M_{1}}, M_{1} \in \mathscr{L}(M)$, we shall write

$$
d_{M}^{G}\left(M_{1}, L\right)=d_{M}^{G}(\mathfrak{b}, L) .
$$

Fix a small point $\xi$ in $\mathfrak{a}_{M}^{\mathfrak{b}}$, and consider an element $L \in \mathscr{L}(M)$ with

$$
\operatorname{dim}\left(\mathfrak{a}_{M}^{\mathfrak{b}}\right)+\operatorname{dim}\left(\mathfrak{a}_{M}^{L}\right) \geq \operatorname{dim}\left(\mathfrak{a}_{M}^{G}\right) .
$$

Assume that $\xi$ is in general position in $\mathfrak{a}_{M}^{\mathfrak{b}}$. Then the affine space $\xi+\mathfrak{b}^{G}$ does not intersect $\mathfrak{a}_{L}^{G}$ unless $\mathfrak{a}_{M}^{G}=\mathfrak{a}_{M}^{\mathfrak{b}} \oplus \mathfrak{a}_{M}^{L}$, or equivalently, unless $d_{M}^{G}(\mathfrak{b}, L) \neq 0$. 
In this case, the spaces $\xi+\mathfrak{b}^{G}$ and $\mathfrak{a}_{L}^{G}$ intersect at one point. The point is nonsingular, and so belongs to a chamber $\mathfrak{a}_{Q}^{+}$, for a unique element $Q=Q_{L}$ in $\mathscr{P}(L)$. Thus, $\xi$ determines a section

$$
L \rightarrow Q_{L}
$$

from the set

$$
\left\{L \in \mathscr{L}(M): d_{M}^{G}(\mathfrak{b}, L) \neq 0\right\}
$$

into the fibers $\mathscr{P}(L)$.

Proposition 7.1. Suppose that

$$
c_{P}(\lambda), \quad P \in \mathscr{P}(M), \lambda \in i \mathrm{a}_{M}^{*},
$$

is a $(G, M)$-family. Then for any $\nu \in i \mathfrak{b}^{*}$, we have

$$
c_{\mathfrak{b}}(\nu)=\sum_{L \in \mathscr{L}(M)} d_{M}^{G}(\mathfrak{b}, L) c_{M}^{Q_{L}}(\nu) .
$$

The proof of this proposition requires a study of convex polytopes. In order not to interrupt the discussion, we shall postpone the proof until the appendix. In the rest of this section, we shall derive some simple consequences of the proposition.

Most of the applications of the proposition concern only the case $\nu=0$, so we state this separately.

Corollary 7.2. $c_{\mathfrak{b}}=\sum_{L \in \mathscr{L}(M)} d_{M}^{G}(\mathfrak{b}, L) c_{M}^{Q_{L}}$.

For certain natural $(G, M)$-families, Corollary 7.2 provides a formula which is independent of the section $L \rightarrow Q_{L}$.

Corollary 7.3. Suppose that for any $L \in \mathscr{L}(M)$, the number

$$
c_{M}^{L}=c_{M}^{Q}, \quad Q \in \mathscr{P}(L),
$$

is independent of $Q$. Then

$$
c_{\mathfrak{b}}=\sum_{L \in \mathscr{L}(M)} d_{M}^{G}(\mathfrak{b}, L) c_{M}^{L} .
$$

Another special case of Corollary 7.2 pertains to products of $(G, M)$-families. Instead of $(G, M)$, we take the pair

$$
(\mathscr{G}, \mathscr{M})=(G \times G, M \times M) \text {. }
$$

Then $\mathfrak{a}_{\mathscr{M}}=\mathfrak{a}_{M} \oplus \mathfrak{a}_{M}$, and $\mathscr{L}(\mathscr{M})$ consists of the set of pairs

$$
\mathscr{L}=\left(L_{1}, L_{2}\right), \quad L_{i} \in \mathscr{L}(M) .
$$

Take $\mathfrak{b}$ to be the direct sum of $\mathfrak{a}_{\mathscr{G}}$ with the space $\mathfrak{a}_{M}$, embedded diagonally in $\mathfrak{a}_{\mathscr{K}}$. It is a special subspace. In order to apply the proposition, we must fix a small point

$$
\Xi=(H,-H), \quad H \in \mathfrak{a}_{M},
$$


in general position in the orthogonal complement of $\mathfrak{b}$. For any pair $\mathscr{L}=$ $\left(L_{1}, L_{2}\right)$ in $\mathscr{L}(\mathscr{M})$, one checks that

$$
d_{\mathscr{M}}^{\mathscr{G}}(\mathfrak{b}, L)=2^{(1 / 2) \operatorname{dim}\left(\mathfrak{a}_{M}^{G}\right)} d_{M}^{G}\left(L_{1}, L_{2}\right) .
$$

If this number is nonzero, we have

$$
\mathfrak{a}_{M}^{G}=\mathfrak{a}_{M}^{L_{1}} \oplus \mathfrak{a}_{M}^{L_{2}}=\mathfrak{a}_{L_{1}}^{G} \oplus \mathfrak{a}_{L_{2}}^{G},
$$

and we can write

$$
H=\frac{1}{2} H_{1}-\frac{1}{2} H_{2}, \quad H_{1} \in \mathfrak{a}_{L_{1}}^{G}, H_{2} \in \mathfrak{a}_{L_{2}}^{G} .
$$

For each $i=1,2, H_{i}$ is a point in general position in $\mathfrak{a}_{L_{i}}^{G}$, and belongs to a chamber $\mathfrak{a}_{Q_{i}}^{+}$, for a unique element $Q_{i} \in \mathscr{P}\left(L_{i}\right)$. Then $\left(L_{1}, L_{2}\right) \rightarrow\left(Q_{1}, Q_{2}\right)$ is the section determined by the point $\Xi$. Suppose that $\left\{c_{P}(\lambda)\right\}$ and $\left\{d_{P}(\lambda)\right\}$ are two $(G, M)$-families. Then

$$
c_{\mathscr{P}}(\Lambda)=c_{P_{1}}\left(\lambda_{1}\right) d_{P_{2}}\left(\lambda_{2}\right), \quad \mathscr{P} \in \mathscr{P}(\mathscr{M}), \Lambda \in i \mathfrak{a}_{\mathscr{M}}^{*},
$$

is a $(\mathscr{G}, \mathscr{M})$-family, where

$$
\mathscr{P}=\left(P_{1}, P_{2}\right), \quad P_{1}, P_{2} \in \mathscr{P}(M),
$$

and

$$
\Lambda=\left(\lambda_{1}, \lambda_{2}\right), \quad \lambda_{1}, \lambda_{2} \in i \mathfrak{a}_{M}^{*} .
$$

Its restriction to $\mathfrak{b}$ is just

$$
(c d)_{P}(\lambda)=c_{P}(\lambda) d_{P}(\lambda), \quad P \in \mathscr{P}(M), \lambda \in i \mathfrak{a}_{M}^{*},
$$

the product $(G, M)$-family. One checks that

$$
\theta_{\mathscr{P}}(\lambda, \lambda)=2^{(1 / 2) \operatorname{dim}\left(\mathfrak{a}_{M}^{G}\right)} \theta_{P}(\lambda),
$$

if $\mathscr{P}$ corresponds to the point $(P, P)$. Corollary 7.2 becomes

Corollary 7.4.

$$
(c d)_{M}=\sum_{L_{1}, L_{2} \in \mathscr{L}(M)} d_{M}^{G}\left(L_{1}, L_{2}\right) c_{M}^{Q_{1}} d_{M}^{Q_{2}}
$$

where $\left(Q_{1}, Q_{2}\right)$ stands for the value of the section at $\left(L_{1}, L_{2}\right)$.

Corollary 7.4 is reminiscent of earlier product formulas for $(G, M)$-families, and in particular, Lemma 6.3 of [1(a)]. It seems to be independent of this result, but it does imply Corollary 6.5 of [1(a)], which is a special case. Suppose that $\left\{c_{P}(\lambda)\right\}$ satisfies the condition of Corollary 7.3. The formula in Corollary 7.4 contains a sum over pairs $\left(L_{1}, L_{2}\right)$, with $L_{i} \in \mathscr{P}(M)$, such that $d_{M}^{G}\left(L_{1}, L_{2}\right) \neq$ 0 . We shall fix $L=L_{1}$ and use Corollary 7.3 with $\mathfrak{b}=\mathfrak{a}_{L_{1}}$ to interpret the remaining sums over $L_{2}$. Take $\xi$ to be the projection of $(-2 H)$ onto the orthogonal complement of $\mathfrak{a}_{L_{1}}^{G}$ in $\mathfrak{a}_{M}^{G}$. Then

$$
\xi+\mathfrak{b}=-2 H+\mathfrak{a}_{L_{1}}=H_{2}+\mathfrak{a}_{L_{1}} .
$$


This intersects $\mathfrak{a}_{L_{2}}^{G}$ in the unique point $H_{2}$. But for a given $L_{2}, Q_{2}$ is the unique element in $\mathscr{P}\left(L_{2}\right)$ such that $H_{2}$ belongs to $\mathfrak{a}_{Q_{2}}^{+}$. Combining Corollaries 7.3 and 7.4 , we obtain

This is Corollary 6.5 of $[1(a)]$.

$$
(c d)_{M}=\sum_{L \in \mathscr{L}(M)} d_{M}^{L} d_{L}
$$

We shall conclude this paragraph with some supplementary remarks on the Jacobians $d_{M}^{G}(\mathfrak{b}, L)$. Suppose that $M_{1} \in \mathscr{L}(M)$ is fixed and that $\mathfrak{b}$ is a special subspace of $\mathfrak{a}_{M_{1}}$. Suppose that $\left\{c_{P}(\lambda)\right\}$ is a $(G, M)$-family that satisfies the condition of Corollary 7.3. We can apply Corollary 7.3 in two stages, first with $M_{1}$ as the base, and then with $M$ itself. We obtain

$$
\begin{aligned}
c_{\mathfrak{b}} & =\sum_{L_{1} \in \mathscr{L}\left(M_{1}\right)} d_{M_{1}}^{G}\left(\mathfrak{b}, L_{1}\right) c_{M_{1}}^{L_{1}} \\
& =\sum_{L_{1} \in \mathscr{L}\left(M_{1}\right)} d_{M_{1}}^{G}\left(\mathfrak{b}, L_{1}\right) \sum_{L \in \mathscr{L}^{L_{1}}(M)} d_{M}^{L_{1}}\left(M_{1}, L\right) c_{M}^{L} .
\end{aligned}
$$

Let us agree to set $d_{M}^{L_{1}}\left(M_{1}, L\right)=0$ if $L_{1}$ does not contain both $M_{1}$ and $L$. Then

$$
c_{\mathfrak{b}}=\sum_{L \in \mathscr{L}(M)}\left(\sum_{L_{1} \in \mathscr{L}\left(M_{1}\right)} d_{M}^{L_{1}}\left(M_{1}, L\right) d_{M_{1}}^{G}\left(\mathfrak{b}, L_{1}\right)\right) c_{M}^{L} .
$$

On the other hand, the direct application of Corollary 7.3 gives

$$
c_{\mathfrak{b}}=\sum_{L \in \mathscr{L}(M)} d_{M}^{G}(\mathfrak{b}, L) c_{M}^{L} .
$$

We can choose $\left\{c_{P}(\lambda)\right\}$ so as to compare the coefficients of these two expressions. Fix an element $L \in \mathscr{L}(M)$ with $\operatorname{dim}\left(\mathfrak{a}_{M}^{L}\right)=\operatorname{dim}\left(\mathfrak{b}^{G}\right)$, and set

$$
c_{P}(\lambda)=\prod_{\alpha \in \Sigma_{P}^{r}} c_{\alpha}\left(\lambda\left(\alpha^{\vee}\right)\right), \quad \lambda \in i \mathfrak{a}_{M}^{*}, P \in \mathscr{P}(M),
$$

where $\Sigma_{P}^{r}$ stands for the set of reduced roots of $\left(G, A_{M}\right)$, and

$$
c_{\alpha}(z)= \begin{cases}e^{z}, & \text { if } \alpha \text { vanishes on } \mathfrak{a}_{L}, \\ 1, & \text { otherwise. }\end{cases}
$$

Then $\left\{c_{p}(\lambda)\right\}$ is a $(G, M)$-family which satisfies the condition of Corollary 7.3. It is easy to see that if $L^{\prime}$ is any element in $\mathscr{L}(M)$ with $\operatorname{dim}\left(\mathfrak{a}_{M}^{L^{\prime}}\right)=\operatorname{dim}\left(\mathfrak{b}^{G}\right)$, then $c_{M}^{L^{\prime}}$ vanishes unless $L^{\prime}=L$. It follows that $L$ gives the only nonvanishing summand in the two expansions for $c_{\mathfrak{b}}$. We obtain

$$
d_{M}^{G}(\mathfrak{b}, L)=\sum_{L_{1} \in \mathscr{L}\left(M_{1}\right)} d_{M}^{L_{1}}\left(M_{1}, L\right) d_{M_{1}}^{G}\left(\mathfrak{b}, L_{1}\right) .
$$

There is a variant of this formula which we shall need. Fix a special subspace $b \subset \mathfrak{a}_{M}$. Proposition 7.1 and its corollaries have versions for arbitrary $\left(\mathfrak{a}_{G}, b\right)$ families. They are all established by the same arguments. In particular, if 
$\left\{c_{P}(\lambda)\right\}$ and $\left\{d_{P}(\lambda)\right\}$ are $(G, M)$-families which both satisfy the conditions of Corollary 7.3, the remarks following Corollary 7.4 can be adapted to the resulting $\left(\mathfrak{a}_{G}, \mathfrak{b}\right)$ families. The result is

$$
(c d)_{\mathfrak{b}}=\sum_{\mathfrak{b}_{1} \in \mathscr{L}(\mathfrak{b})} c_{\mathfrak{b}}^{\mathfrak{b}_{1}} d_{\mathfrak{b}_{1}} .
$$

Apply Corollary 7.3 to the left-hand side of this. We obtain

$$
\begin{aligned}
(c d)_{\mathfrak{b}} & =\sum_{L \in \mathscr{L}(M)} d_{M}^{G}(\mathfrak{b}, L)(c d)_{M}^{L} \\
& =\sum_{L \in \mathscr{L}(M)} \sum_{M_{1} \in \mathscr{L}^{L}(M)} d_{M}^{G}(\mathfrak{b}, L) c_{M}^{M_{1}} d_{M_{1}}^{L} .
\end{aligned}
$$

We can also apply Corollary 7.3 to the right-hand side. If $\mathfrak{b}_{1}$ is contained in $\mathfrak{a}_{M_{1}}$, we define $d_{M}^{\mathfrak{b}_{1}}\left(\mathfrak{b}, M_{1}\right)$ exactly as we defined $d_{M}^{G}\left(\mathfrak{b}, M_{1}\right)$, but with $\mathfrak{a}_{G}$ replaced by $\mathfrak{b}_{1}$. If $\mathfrak{b}_{1}$ is not contained in $\mathfrak{a}_{M_{1}}$, we simply set $d_{M}^{\mathfrak{b}_{1}}\left(\mathfrak{b}, M_{1}\right)=0$. Note that if $G$ is replaced by $G_{1}=M_{\mathfrak{b}_{1}}$, then $\mathfrak{b}+\mathfrak{a}_{G_{1}}$ becomes a special subspace of $\mathfrak{a}_{M}$, and one has

$$
d_{M}^{\mathfrak{b}_{1}}\left(\mathfrak{b}, M_{1}\right)=d_{M}^{G_{1}}\left(\mathfrak{b}+\mathfrak{a}_{G_{1}}, M_{1}\right) .
$$

Applied in this context, Corollary 7.3 is easily converted to the formula

$$
c_{\mathfrak{b}}^{\mathfrak{b}_{1}}=\sum_{M_{1} \in \mathscr{L}(M)} d_{M}^{\mathfrak{b}_{1}}\left(\mathfrak{b}, M_{1}\right) c_{M}^{M_{1}} .
$$

Therefore, the right-hand side equals

$$
\begin{aligned}
& \sum_{\mathfrak{b}_{1} \in \mathscr{L}(\mathfrak{b})} \sum_{M_{1} \in \mathscr{L}(M)} d_{M}^{\mathfrak{b}_{1}}\left(\mathfrak{b}, M_{1}\right) c_{M}^{M_{1}} d_{\mathfrak{b}_{1}} \\
& \quad=\sum_{\mathfrak{b}_{1}} \sum_{M_{1}} d_{M}^{\mathfrak{b}_{1}}\left(\mathfrak{b}, M_{1}\right) c_{M}^{M_{1}} \sum_{L \in \mathscr{L}\left(M_{1}\right)} d_{M_{1}}^{G}\left(\mathfrak{b}_{1}, L\right) d_{M_{1}}^{L} \\
& \quad=\sum_{M_{1} \in \mathscr{L}(M)} \sum_{L \in \mathscr{L}\left(M_{1}\right)}\left(\sum_{\mathfrak{b}_{1} \in \mathscr{L}(\mathfrak{b})} d_{M}^{\mathfrak{b}_{1}}\left(\mathfrak{b}, M_{1}\right) d_{M_{1}}^{G}\left(\mathfrak{b}_{1}, L\right)\right) c_{M}^{M_{1}} d_{M_{1}}^{L} .
\end{aligned}
$$

Arguing as above, one can see without much trouble how to choose $\left\{c_{P}(\lambda)\right\}$ and $\left\{d_{P}(\lambda)\right\}$ so as to isolate any given pair of coefficients. Equating the coefficients, one obtains

$$
d_{M}^{G}(\mathfrak{b}, L)=\sum_{\mathfrak{b}_{1} \in \mathscr{L}(\mathfrak{b})} d_{M}^{\mathfrak{b}_{1}}\left(\mathfrak{b}, M_{1}\right) d_{M_{1}}^{G}\left(\mathfrak{b}_{1}, L\right)
$$

\section{DESCENT}

We want to establish descent formulas for our various distributions. For example, if $M_{1} \in \mathscr{L}(M)$ and $\gamma$ is a $G$-regular element in $M\left(F_{S}\right)$, then Lemma 10.3 of [1(a)] provides a formula for $I_{M_{1}}(\gamma, f)$ in terms of the distributions 
$\widehat{I}_{M}^{L}\left(\gamma, f_{L}\right)$. This formula, however, does not apply to arbitrary elements in $M\left(F_{S}\right)$. The correct generalization must be stated in terms of induced ( onjugacy classes. For any $\gamma \in M\left(F_{S}\right)$, recall that $\gamma^{M_{1}}$ denotes the induced space in $M_{1}\left(F_{S}\right)$. If $\gamma$ is such that $M_{1, \gamma}=M_{\gamma}$, then $\gamma^{M_{1}}$ is just the $M_{1}^{0}\left(F_{S}\right)$-orbit of $\gamma$. In general, however, $\gamma^{M_{1}}$ is a finite union of $M_{1}^{0}\left(F_{S}\right)$-orbits $\left\{\gamma_{i}\right\}$ in $M_{1}\left(F_{S}\right)$. We shall prove a formula for

$$
I_{M_{1}}\left(\gamma^{M_{1}}, f\right)=\sum_{i} I_{M_{1}}\left(\gamma_{i}, f\right)
$$

in terms of the distributions $\widehat{I}_{M}^{L}\left(\gamma, f_{L}\right)$.

We shall in fact establish a more general result. Suppose that $\mathfrak{b}$ is a special subspace of $\mathfrak{a}_{M}$. In $\S 7$ we defined the Levi set $M_{\mathfrak{b}} \in \mathscr{L}(M)$. If $\gamma_{1}$ belongs to $M_{\mathfrak{b}}\left(F_{S}\right)$, we can define the distribution $J_{\mathfrak{b}}\left(\gamma_{1}, f\right)$ on $\mathscr{H}_{\text {ac }}\left(G\left(F_{S}\right)\right)$ exactly as in the special case that $\mathfrak{b}=\mathfrak{a}_{M}$. (See [1(d), (2.1) and (6.5)].) We need only replace the volume $v_{M}(x)$ in [1(d), (2.1)] by $v_{\mathfrak{b}}(x)$, the volume in $\mathfrak{b}^{G}$ of the convex hull of

$$
\left\{H_{Q}(x): Q \in \mathscr{P}\left(M_{\mathfrak{b}}\right), \mathfrak{a}_{Q}^{+} \cap \mathfrak{b} \neq \varnothing\right\} .
$$

Similarly, copying the definition of $\phi_{M}$ [1(e),§7], we can introduce a map

$$
\phi_{\mathrm{b}}: \mathscr{H}_{\mathrm{ac}}\left(G\left(F_{S}\right)\right) \rightarrow \mathscr{I}_{\mathrm{ac}}\left(M_{\mathrm{b}}\left(F_{S}\right)\right) .
$$

Some effort was required in [1(d)] and [1(e)] to establish the existence and properties of $J_{M}$ and $\phi_{M}$. However, the identical arguments suffice for arbitrary $\mathfrak{b}$; alternatively, one can deal with the general case directly from that of $M=M_{\mathfrak{b}}$ by applying Proposition 7.1. At any rate, we shall take for granted the obvious analogues of results and constructions that have hitherto been discussed only in the case that $\mathfrak{b}$ equals $\mathfrak{a}_{M}$. In particular, we have an invariant distribution $I_{b}\left(\gamma_{1}\right)$ on $\mathscr{H}_{\text {ac }}\left(G\left(F_{S}\right)\right)$. It is defined inductively by

$$
I_{\mathfrak{b}}\left(\gamma_{1}, f\right)=J_{\mathfrak{b}}\left(\gamma_{1}, f\right)-\sum_{\mathfrak{b}_{1} \in \mathscr{L}_{0}(\mathfrak{b})} \hat{I}_{\mathfrak{b}}^{\mathfrak{b}_{1}}\left(\gamma_{1}, \phi_{\mathfrak{b}_{1}}(f)\right),
$$

where

$$
I_{\mathfrak{b}}^{\mathfrak{b}_{1}}\left(\gamma_{1}\right)=I_{\mathfrak{b}+\mathfrak{a}_{G_{1}}}^{G_{1}}\left(\gamma_{1}\right), \quad G_{1}=M_{\mathfrak{b}_{1}} .
$$

Included in the definition is the induction assumption that for any $\mathfrak{b}_{1} \in \mathscr{L}_{0}(\mathfrak{b})$, the distribution $I_{\mathfrak{b}}^{\mathfrak{b}_{1}}\left(\gamma_{1}\right)$ on $\mathscr{H}_{\mathrm{ac}}\left(G_{1}\left(F_{S}\right)\right)$ is supported on characters. The next theorem will provide a formula which resolves this new induction hypothesis in terms of the original one.

The space $\mathfrak{b}$ is always contained in $\mathfrak{a}_{M_{\mathfrak{b}}}$. If the two spaces are the same, then $I_{b}\left(\gamma_{1}, f\right)$ is just equal to $I_{M_{\mathfrak{b}}}\left(\gamma_{1}, f\right)$. However, this need not always be so. For example, $M_{\mathfrak{b}}$ could be defined over a subfield $F_{1}$ of $F$, and $\mathfrak{b}$ could be the split component of $M_{\mathfrak{b}}$ over $F_{1}$. This might well be a proper subspace of the split component $\mathfrak{a}_{M_{\mathfrak{b}}}$ of $M_{\mathfrak{b}}$ over $F$, in which case $I_{\mathfrak{b}}\left(\gamma_{1}, f\right)$ would not be equal to $I_{M_{\mathrm{b}}}\left(\gamma_{1}, f\right)$. 
If $\gamma$ belongs to $M\left(F_{S}\right)$, write $\gamma^{\mathfrak{b}}=\gamma^{M_{\mathfrak{b}}}$ for the induced class in $M_{\mathfrak{b}}\left(F_{S}\right)$, and set

$$
I_{\mathfrak{b}}\left(\gamma^{\mathfrak{b}}, f\right)=I_{\mathfrak{b}}\left(\gamma^{M_{\mathfrak{b}}}, f\right) .
$$

Theorem 8.1. Given $\gamma \in M\left(F_{S}\right)$, we have

$$
I_{\mathfrak{b}}\left(\gamma^{\mathfrak{b}}, f\right)=\sum_{L \in \mathscr{L}(M)} d_{M}^{G}(\mathfrak{b}, L) \widehat{I}_{M}^{L}\left(\gamma, f_{L}\right), \quad f \in \mathscr{H}_{\mathrm{ac}}\left(G\left(F_{S}\right)\right) .
$$

Proof. Both sides depend only on the values of $f$ on

$$
\left\{x \in G\left(F_{S}\right): H_{G}(x)=H_{G}(\gamma)\right\} .
$$

Since the restriction of $f$ to this subset coincides with that of some function in $\mathscr{H}\left(G\left(F_{S}\right)\right)$, we can assume that $f$ itself belongs to $\mathscr{H}\left(G\left(F_{S}\right)\right)$. We shall also assume for the moment that $\gamma \in M\left(F_{S}\right)$ is such that $M_{\gamma}=G_{\gamma}$. Then $\gamma^{b}$ equals $\gamma$ and $J_{\mathfrak{b}}(\gamma, f)$ equals

$$
\left|D^{G}(\gamma)\right|^{1 / 2} \int_{G_{\gamma}\left(F_{S}\right) \backslash G^{0}\left(F_{S}\right)} f\left(x^{-1} \gamma x\right) v_{\mathfrak{b}}(x) d x .
$$

Applying Corollary 7.2 to the $(G, M)$-family

$$
\left\{v_{P}(x)=e^{-\lambda\left(H_{P}(x)\right)}: P \in \mathscr{P}(M)\right\},
$$

we write

$$
v_{\mathfrak{b}}(x)=\sum_{L \in \mathscr{L}(M)} d_{M}^{G}(\mathfrak{b}, L) v_{M}^{L_{Q}}(x) .
$$

This allows us to make a standard change of variables in the integral over $G_{\gamma}\left(F_{S}\right) \backslash G^{0}\left(F_{S}\right)[1(\mathrm{~d}),(8.11)]$, and we find that $J_{\mathfrak{b}}(\gamma, f)$ equals

$$
\sum_{L \in \mathscr{L}(M)} d_{M}^{G}(\mathfrak{b}, L) J_{M}^{L}\left(\gamma, f_{Q_{L}}\right)
$$

Our distribution $I_{\mathfrak{b}}(\gamma, f)$ equals the difference between (8.2) and the expression

$$
\sum_{\mathfrak{b}_{1} \in \mathscr{L}_{0}(\mathfrak{b})} \hat{I}_{\mathfrak{b}}^{b_{1}}\left(\gamma, \phi_{\mathfrak{b}_{1}}(f)\right)
$$

We can assume inductively that the theorem holds for each of the distributions $I_{b}^{\mathfrak{b}_{1}}(\gamma)$. Then (8.3) may be written

$$
\sum_{\mathfrak{b}_{1} \in \mathscr{L}_{0}(\mathfrak{b})} \sum_{M_{1} \in \mathscr{L}^{\mathfrak{b}_{1}}(M)} d_{M}^{\mathfrak{b}_{1}}\left(\mathfrak{b}, M_{1}\right) \widehat{I}_{M}^{M_{1}}\left(\gamma, \phi_{\mathfrak{b}_{1}}(f)_{M_{1}}\right) .
$$

Now $\phi_{\mathfrak{b}_{1}}(f)_{M_{1}}$ is a function in $\mathscr{J}_{\mathrm{ac}}\left(M_{1}\left(F_{S}\right)\right)$. Its value at any representation $\pi_{1} \in \Pi_{\text {temp }}\left(M_{1}\left(F_{S}\right)\right)$ equals

$$
\operatorname{tr}\left(\mathscr{R}_{\mathfrak{b}_{1}}\left(\pi_{1}^{\mathfrak{b}_{1}}, Q_{0}\right) \mathscr{I}_{Q_{0}}\left(\pi_{1}^{\mathfrak{b}_{1}}, f\right)\right) .
$$


Here $Q_{0}$ is a fixed element in $\mathscr{P}\left(G_{1}\right)$, where $G_{1}=M_{\mathfrak{b}_{1}}$, and $\pi_{1}^{\mathfrak{b}_{1}}$ stands for the induced representation $\pi_{1}^{G_{1}}$; the operator $\mathscr{R}_{\mathfrak{b}_{1}}\left(\pi_{1}^{\mathfrak{b}_{1}}, Q_{0}\right)$ is obtained from the restriction to $\mathfrak{b}_{1}$ of the $\left(G, G_{1}\right)$-family

$$
\mathscr{R}_{Q}\left(\nu, \pi_{1}^{\mathfrak{b}_{1}}, Q_{0}\right), \quad Q \in \mathscr{P}\left(G_{1}\right), \nu \in i \mathfrak{a}_{G_{1}}^{*},
$$

described in $\S 6$ of [1(e)]. It follows easily from Corollary 7.2 that

$$
\phi_{\mathfrak{b}_{1}}(f)_{M_{1}}=\sum_{L \in \mathscr{L}\left(M_{1}\right)} d_{M_{1}}^{G}\left(\mathfrak{b}_{1}, L\right) \phi_{M_{1}}^{L}\left(f_{Q_{L}}\right)
$$

(See also formula (7.8) of [1(a)].) Therefore (8.3) equals

$$
\sum_{M_{1} \in \mathscr{L}(M)} \sum_{L \in \mathscr{L}\left(M_{1}\right)} \sum_{\mathfrak{b}_{1} \in \mathscr{L}_{0}(\mathfrak{b})} d_{M}^{\mathfrak{b}_{1}}(\mathfrak{b}, M) d_{M_{1}}^{G}\left(\mathfrak{b}_{1}, L\right) \widehat{L}_{M}^{M_{1}}\left(\gamma, \phi_{M_{1}}^{L}\left(f_{Q_{L}}\right)\right) .
$$

The section $L \rightarrow Q_{L}$ is defined in (8.4) with respect to some point $\xi_{1} \in \mathfrak{a}_{M_{1}}^{\mathfrak{b}_{1}}$ in general position, while in (8.2) it is defined with respect to a point $\xi \in \mathfrak{a}_{M}^{\mathfrak{b}}$. However, it turns out that the notation is consistent. For we need only consider elements $M_{1}$ such that $d_{M}^{\mathfrak{b}_{1}}\left(\mathfrak{b}, M_{1}\right) \neq 0$. This means that

$$
\mathfrak{a}_{M}^{\mathfrak{b}_{1}}=\mathfrak{a}_{M_{1}}^{\mathfrak{b}_{1}} \oplus \mathfrak{b}^{\mathfrak{b}_{1}}
$$

and so there is a natural isomorphism

$$
\mathfrak{a}_{M}^{\mathfrak{b}} \cong \mathfrak{a}_{M} / \mathfrak{b} \stackrel{\simeq}{\longrightarrow} \mathfrak{a}_{M_{1}} / \mathfrak{b}_{1} \cong \mathfrak{a}_{M_{1}}^{\mathfrak{b}_{1}}
$$

We take $\xi_{1}$ to be the image of $\xi$. Then if $L$ is any element in $\mathscr{L}\left(M_{1}\right)$ with $d_{M_{1}}^{G}\left(\mathfrak{b}_{1}, L\right) \neq 0$, we have

$$
\mathfrak{a}_{M_{1}}^{G}=\mathfrak{a}_{L}^{G} \oplus \mathfrak{b}_{1}^{G},
$$

and $\xi_{1}+\mathfrak{b}_{1}$ and $\xi+\mathfrak{b}$ both intersect $\mathfrak{a}_{L}^{G}$ at the same point. Consequently for any given $L$, the parabolic $Q_{L}$ in (8.4) is the same as that in (8.2). In particular, $Q_{L}$ is independent of $\mathfrak{b}_{1}$. Thus, the only part of the expression (8.4) which depends on $\mathfrak{b}_{1}$ is the sum

$$
\sum_{\mathfrak{b}_{1} \in \mathscr{L}_{0}(\mathfrak{b})} d_{M}^{\mathfrak{b}_{1}}\left(\mathfrak{b}, M_{1}\right) d_{M_{1}}^{G}\left(\mathfrak{b}_{1}, L\right) .
$$

This can be simplified. If $L \neq M_{1}$, we can replace $\mathscr{L}_{0}(\mathfrak{b})$ by $\mathscr{L}(\mathfrak{b})$, for the term corresponding to $\mathfrak{b}_{1}=\mathfrak{a}_{G}$ vanishes. By (7.2), the sum is equal to $d_{M}^{G}(\mathfrak{b}, L)$. If $L=M_{1}$,

$$
d_{M_{1}}^{G}\left(\mathfrak{b}_{1}, L\right)=d_{M_{1}}^{G}\left(\mathfrak{b}_{1}, M_{1}\right)=0,
$$

since $\mathfrak{b}_{1} \neq \mathfrak{a}_{G}$, so in this case the summands are all zero. It follows that (8.4), which on the one hand equals the original expression (8.3), also equals

$$
\sum_{L \in \mathscr{L}(M)} \sum_{\left\{M_{1} \in \mathscr{L}^{L}(M): M_{1} \neq L\right\}} d_{M}^{G}(\mathfrak{b}, L) \widehat{I}_{M}^{M_{1}}\left(\gamma, \phi_{M_{1}}^{L}\left(f_{Q_{L}}\right)\right) .
$$


This is easily combined with (8.2). From the inductive definition of $I_{M}^{L}(\gamma)$ we see that the difference between (8.2) and (8.3) equals

$$
\sum_{L \in \mathscr{L}(M)} d_{M}^{G}(\mathfrak{b}, L) I_{M}^{L}\left(\gamma, f_{Q_{L}}\right) .
$$

Since $\left(f_{Q_{L}}\right)_{L}$ equals $f_{L}$, this becomes

$$
\sum_{L \in \mathscr{L}(M)} d_{M}^{G}(\mathfrak{b}, L) \widehat{I}_{M}^{L}\left(\gamma, f_{L}\right),
$$

the required formula for $I_{\mathfrak{b}}(\gamma, f)$.

Now, suppose that $\gamma$ is an arbitrary element in $M\left(F_{S}\right)$. As in $\left(2.2^{*}\right)$, we can write

$$
I_{\mathfrak{b}}\left(\gamma^{\mathfrak{b}}, f\right)=\lim _{a \rightarrow 1} \sum_{\mathfrak{b}_{1} \in \mathscr{L}(\mathfrak{b})} r_{\mathfrak{b}}^{\mathfrak{b}_{1}}(\gamma, a) I_{\mathfrak{b}_{1}}(a \gamma, f),
$$

where $a$ approaches 1 through the regular points in $A_{M}\left(F_{S}\right)$. The theorem will be established by arguing as in the derivation of (7.2). For the function $r_{\mathrm{b}}^{b_{1}}(\gamma, a)$ comes from a $(G, M)$-family

$$
r_{P}(\lambda, \gamma, a), \quad P \in \mathscr{P}(M), \lambda \in i \mathfrak{a}_{M}^{*},
$$

which satisfies the condition of Corollary 7.3. (See [1(d), Lemma 5.1].) Moreover, we are assuming that $a \in A_{M}\left(F_{S}\right)$ is regular, so that $M_{a \gamma}=G_{a \gamma}$. Applying Corollary 7.3 and what we have just proved, we obtain

$$
\begin{aligned}
& \sum_{\mathfrak{b}_{1} \in \mathscr{L}(\mathfrak{b})} r_{\mathfrak{b}}^{\mathfrak{b}_{1}}(\gamma, a) I_{\mathfrak{b}_{1}}(a \gamma, f) \\
& =\sum_{\mathfrak{b}_{1} \in \mathscr{L}(\mathfrak{b})} \sum_{M_{1} \in \mathscr{L}(M)} d_{M}^{\mathfrak{b}_{1}}\left(\mathfrak{b}, M_{1}\right) r_{M}^{M_{1}}(\gamma, a) I_{\mathfrak{b}_{1}}(a \gamma, f) \\
& =\sum_{\mathfrak{b}_{1}} \sum_{M_{1}} d_{M}^{\mathfrak{b}_{1}}\left(\mathfrak{b}, M_{1}\right) r_{M}^{M_{1}}(\gamma, a) \sum_{L \in \mathscr{L}\left(M_{1}\right)} d_{M_{1}}^{G}\left(\mathfrak{b}_{1}, L\right) \widehat{I}_{M_{1}}^{L}\left(a \gamma, f_{L}\right) \\
& =\sum_{L \in \mathscr{L}(M)} d_{M}^{G}(\mathfrak{b}, L) \sum_{M_{1} \in \mathscr{L}^{L}(M)} r_{M}^{M_{1}}(\gamma, a) \widehat{I}_{M_{1}}^{L}\left(a \gamma, f_{L}\right) .
\end{aligned}
$$

The last step follows from (7.2). But

$$
\lim _{a \rightarrow 1} \sum_{M_{1} \in \mathscr{L}^{L}(M)} r_{M}^{M_{1}}(\gamma, a) \widehat{I}_{M_{1}}^{L}\left(a \gamma, f_{L}\right)=\widehat{I}_{M}^{L}\left(\gamma, f_{L}\right),
$$

by (2.2). Taking the limits in $a$ thus gives us

$$
I_{\mathfrak{b}}\left(\gamma^{\mathfrak{b}}, f\right)=\sum_{L \in \mathscr{L}(M)} d_{M}^{G}(\mathfrak{b}, L) \widehat{I}_{M}^{L}\left(\gamma, f_{L}\right) .
$$

This completes the proof.

We are of course interested in the special case that $\mathfrak{b}=\mathfrak{a}_{M_{1}}$, for some element $M_{1} \in \mathscr{L}(M)$. 
Corollary 8.2. Given $\gamma \in M\left(F_{S}\right)$, we have

$$
I_{M_{1}}\left(\gamma^{M_{1}}, f\right)=\sum_{L \in \mathscr{L}(M)} d_{M}^{G}\left(M_{1}, L\right) \widehat{I}_{M}^{L}\left(\gamma, f_{L}\right), \quad f \in \mathscr{H}_{\mathrm{ac}}\left(G\left(F_{S}\right)\right) .
$$

Corollary 8.3. Suppose that $\gamma \in M\left(F_{S}\right)$ is such that $M_{\gamma}=M_{1, \gamma}$. Then

$$
I_{M_{1}}(\gamma, f)=\sum_{L \in \mathscr{L}(M)} d_{M}^{G}\left(M_{1}, L\right) \widehat{I}_{M}^{L}\left(\gamma, f_{L}\right)
$$

There is a similar descent property of $I_{M}(\pi, X, f)$. Once again, it is important to work in a slightly broader context. Suppose again that $\mathfrak{b}$ is a special subspace of $\mathfrak{a}_{M}$. If $\pi_{1} \in \Pi\left(M_{\mathfrak{b}}\left(F_{S}\right)\right)$ and $X_{1} \in \mathfrak{a}_{M_{1}, S}$, we can define the distributions $J_{\mathfrak{b}}\left(\pi_{1}, X_{1}, f\right)$ on $\mathscr{H}_{\mathrm{ac}}\left(G\left(F_{S}\right)\right)$ exactly the same way as in the special case that $\mathfrak{b}=\mathfrak{a}_{M}$. (See [1(e), $\left.\left.\S \S 6,7\right].\right)$

We can also define an invariant distribution $I_{b}\left(\pi_{1}, X_{1}\right)$ on $\mathscr{H}_{\mathrm{ac}}\left(G\left(F_{S}\right)\right)$ inductively by

$$
I_{\mathfrak{b}}\left(\pi_{1}, X_{1}, f\right)=J_{\mathfrak{b}}\left(\pi_{1}, X_{1}, f\right)-\sum_{\mathfrak{b}_{1} \in \mathscr{L}_{0}(\mathfrak{b})} \widehat{I}_{\mathfrak{b}}^{\mathfrak{b}_{1}}\left(\pi_{1}, X_{1}, \phi_{\mathfrak{b}_{1}}(f)\right) .
$$

Included in the definition is the induction assumption that $I_{b}^{\mathfrak{b}_{1}}\left(\pi_{1}, X_{1}\right)$ is supported on characters. This will be resolved in terms of our original induction hypothesis by the next theorem (together with Theorem 6.1).

Suppose that $\pi \in \Pi\left(M\left(F_{S}\right)\right)$ and $X \in \mathfrak{a}_{M, S}$. We shall write

$$
J_{\mathfrak{b}}(\pi, X, f)=\int_{i \mathfrak{a}_{M, S}^{*} / \mathfrak{b}_{S}^{*}} J_{\mathfrak{b}}\left(\pi_{\lambda}, h_{\mathfrak{b}}(X), f\right) e^{-\lambda(X)} d \lambda,
$$

for any $f \in \mathscr{H}_{\mathrm{ac}}\left(G\left(F_{S}\right)\right)$. (Here $h_{\mathfrak{b}}(X)$ is the projection of $X$ onto $\mathfrak{b}$. As in [1(e)], we shall often write $\pi_{\lambda}$ when we really mean the induced representation $\pi_{\lambda}^{\mathfrak{b}}=\left(\pi_{\lambda}\right)^{M_{\mathfrak{b}}}$.) The integral clearly depends only on the restriction of $f$ to $G\left(F_{S}\right)^{Z}, Z=h_{G}(X)$. Since this is compactly supported, we can always replace $f$ itself by a compactly supported function. It follows from standard estimates [1(e), (12.7)] that the integral over $\lambda$ is absolutely convergent. Define an invariant distribution $I_{b}(\pi, X)$ on $\mathscr{H}_{\mathrm{ac}}\left(G\left(F_{S}\right)\right)$ inductively by

$$
I_{\mathfrak{b}}(\pi, X, f)=J_{\mathfrak{b}}(\pi, X, f)-\sum_{\mathfrak{b}_{1} \in \mathscr{L}_{0}(\mathfrak{b})} \widehat{I}_{\mathfrak{b}}^{\mathfrak{b}_{1}}\left(\pi, X, \phi_{\mathfrak{b}_{1}}(f)\right) .
$$

It then follows that

$$
I_{\mathfrak{b}}(\pi, X, f)=\int_{i \mathfrak{a}_{M, S}^{*} / i^{*}{ }_{s}^{*}} I_{\mathfrak{b}}\left(\pi_{\lambda}, h_{\mathfrak{b}}(X), f\right) e^{-\lambda(X)} d \lambda,
$$

with the integral converging absolutely.

Theorem 8.4. Given $\pi \in \Pi\left(M\left(F_{S}\right)\right)$ and $X \in \mathfrak{a}_{M, S}$, we have

$$
I_{\mathfrak{b}}(\pi, X, f)=\sum_{L \in \mathscr{L}(M)} d_{M}^{G}(\mathfrak{b}, L) \widehat{I}_{M}^{L}\left(\pi, X, f_{L}\right), \quad f \in \mathscr{H}_{\mathrm{ac}}\left(G\left(F_{S}\right)\right) .
$$


Proof. As above, we can assume that $f$ actually belongs to $\mathscr{H}\left(G\left(F_{S}\right)\right)$. It also happens that we can restrict $\pi$. For as in Lemma 3.2(b), we have

$$
I_{\mathfrak{b}}(\pi, X, f)=\sum_{\mathfrak{p} \in \mathscr{P}(\mathfrak{b})} \omega_{\mathfrak{p}} I_{\mathfrak{b}}\left(\pi_{\varepsilon_{\mathfrak{p}}}, X, f\right) e^{-\varepsilon_{\mathfrak{p}}(X)},
$$

where for each $\mathfrak{p}, \omega_{\mathfrak{p}}=\omega\left(b_{\mathfrak{p}}^{+}, 0\right)$, and $\varepsilon_{\mathfrak{p}}$ denotes a small regular point in the dual chamber $\left(\mathfrak{b}^{*}\right)_{\mathfrak{p}}^{+}$. Suppose that $L \in \mathscr{L}(M)$ is such that $d_{M}^{G}(\mathfrak{b}, L) \neq 0$. Then the canonical map

$$
\mathfrak{b}^{*} / \mathfrak{a}_{G}^{*} \rightarrow \mathfrak{a}_{M}^{*} / \mathfrak{a}_{L}^{*}
$$

is an isomorphism. Each chamber in the second space is, up to a set of measure 0 , a union of images of chambers $\left(\mathfrak{b}^{*}\right)_{\mathfrak{p}}^{+}$. Moreover, for any small regular point $\varepsilon$ in $\mathfrak{a}_{M}^{*}$, the number

$$
I_{M, \varepsilon}(\pi, X, f)=I_{M}\left(\pi_{\varepsilon}, X, f\right) e^{-\varepsilon(X)}
$$

depends only on the chamber in $\mathfrak{a}_{M}^{*}$ which contains $\varepsilon$. Consequently

$$
\begin{aligned}
\sum_{\mathfrak{p} \in \mathscr{P}(\mathfrak{b})} \omega_{\mathfrak{p}} \widehat{I}_{M}^{L}\left(\pi_{\varepsilon_{\mathfrak{p}}}, X, f_{L}\right) e^{-\varepsilon_{\mathfrak{p}}(X)} & =\sum_{R \in \mathscr{P} L(M)} \omega_{R} \widehat{I}_{M, \varepsilon_{R}}^{L}\left(\pi, X, f_{L}\right) \\
& =\widehat{I}_{M}^{L}\left(\pi, X, f_{L}\right) .
\end{aligned}
$$

It follows that if the theorem holds with $\pi$ replaced by $\pi_{\varepsilon_{\mathfrak{b}}}$, it then holds for $\pi$ itself. We may therefore assume that $\pi$ is in general position, as a point in some $\mathfrak{a}_{M}^{*}$-orbit in $\Pi\left(M\left(F_{S}\right)\right)$.

The general position of $\pi$ implies that the function

$$
J_{\mathfrak{b}}\left(\pi_{\lambda}, f\right)=\operatorname{tr}\left(\mathscr{R}_{\mathfrak{b}}\left(\pi_{\lambda}^{\mathfrak{b}}, Q_{0}\right) \mathscr{F}_{Q_{0}}\left(\pi_{\lambda}^{\mathfrak{b}}, f\right)\right)
$$

is analytic for $\lambda \in i \mathrm{a}_{M}^{*}$. Recall that $Q_{0}$ is a fixed element in $\mathscr{P}\left(M_{\mathfrak{b}}\right)$, and $\mathscr{R}_{\mathfrak{b}}\left(\pi_{\lambda}^{\mathfrak{b}}, Q_{0}\right)$ is obtained from the restriction to $\mathfrak{b}$ of a $\left(G, M_{\mathfrak{b}}\right)$-family

$$
\mathscr{R}_{Q}\left(\nu, \pi_{\lambda}^{\mathfrak{b}}, Q_{0}\right), \quad Q \in \mathscr{P}\left(M_{\mathfrak{b}}\right), \nu \in i \mathfrak{a}_{M_{\mathfrak{b}}}^{*} .
$$

As in [1(a), (7.8)] we have

$$
\operatorname{tr}\left(\mathscr{R}_{\mathfrak{b}}\left(\pi_{\lambda}^{\mathfrak{b}}, Q_{0}\right) \mathscr{I}_{Q_{0}}\left(\pi_{\lambda}^{\mathfrak{b}}, f\right)\right)=\operatorname{tr}\left(\mathscr{R}_{\mathfrak{b}}\left(\pi_{\lambda}, P_{0}\right) \mathscr{I}_{P_{0}}\left(\pi_{\lambda}, f\right)\right)
$$

for any fixed element $P_{0} \in \mathscr{P}(M)$. It follows that

$$
\begin{aligned}
J_{\mathfrak{b}}(\pi, X, f) & =\int_{i a_{M, S}^{*} / i_{s}^{*}}\left(\int_{i b_{S}^{*}} J_{\mathfrak{b}}\left(\pi_{\lambda+\mu}, f\right) e^{-\mu\left(h_{\mathfrak{b}}(X)\right)} d \mu\right) e^{-\lambda(X)} d \lambda \\
& =\int_{i \mathfrak{a}_{M, S}^{*}} \operatorname{tr}\left(\mathscr{R}_{\mathfrak{b}}\left(\pi_{\lambda}, P_{0}\right) \mathscr{I}_{P_{0}}\left(\pi_{\lambda}, f\right)\right) e^{-\lambda(X)} d \lambda .
\end{aligned}
$$

If we apply Corollary 7.2 to the $(G, M)$-family

$$
\mathscr{R}_{P}\left(\nu, \pi_{\lambda}, P_{0}\right), \quad P \in \mathscr{P}(M), \nu \in i \mathrm{a}_{M}^{*},
$$


we find that $J_{\mathfrak{b}}(\pi, X, f)$ equals

$$
\sum_{L \in \mathscr{L}(M)} d_{M}^{G}(\mathfrak{b}, L) \int_{i \mathfrak{a}_{M}^{*}, S} \operatorname{tr}\left(\mathscr{R}_{M}^{Q_{L}}\left(\pi_{\lambda}, P_{0}\right) \mathscr{I}_{P_{0}}\left(\pi_{\lambda}, f\right)\right) e^{-\lambda(X)} d \lambda
$$

The argument used to prove Lemma 7.1 of $[1(a)]$ then allows us to write this last expression as

$$
\sum_{L \in \mathscr{L}(M)} d_{M}^{G}(\mathfrak{b}, L) \int_{\mathfrak{i a}_{M, S}^{*}} J_{M}^{L}\left(\pi_{\lambda}, f_{Q_{L}}\right) e^{-\lambda(X)} d \lambda
$$

It follows that $J_{\mathfrak{b}}(\pi, X, f)$ equals

$$
\sum_{L \in \mathscr{L}(M)} d_{M}^{G}(\mathfrak{b}, L) J_{M}^{L}\left(\pi, X, f_{Q_{L}}\right)
$$

Our distribution $I_{\mathfrak{b}}(\pi, X, f)$ equals the difference between (8.6) and the expression

$$
\sum_{\mathfrak{b}_{1} \in \mathscr{L}_{0}(\mathfrak{b})}{\widehat{I_{b}}}_{\mathfrak{b}_{1}}\left(\pi, X, \phi_{\mathfrak{b}_{1}}(f)\right)
$$

The proof is now identical to that of Theorem 8.1. Assuming inductively that Theorem 8.4 holds for the distributions $I_{\mathfrak{b}}^{\mathfrak{b}_{1}}(\pi, X)$, we are lead to an expansion of (8.7) into

$$
\sum_{L \in \mathscr{L}(M)} \sum_{\left\{M_{1} \in \mathscr{L}^{L}(M): M_{1} \neq L\right\}} d_{M}^{G}(\mathfrak{b}, L) \widehat{I}_{M}^{M_{1}}\left(\pi, X, \phi_{M_{1}}^{L}\left(f_{Q_{L}}\right)\right) .
$$

It follows that the difference between (8.6) and (8.7) equals

$$
\sum_{L \in \mathscr{L}(M)} d_{M}^{G}(\mathfrak{b}, L) \widehat{I}_{M}^{L}\left(\pi, X, f_{L}\right)
$$

the required formula for $I_{\mathfrak{b}}(\pi, X, f)$.

Consider the special case that $\mathfrak{b}=\mathfrak{a}_{M_{1}}$ for some element $M_{1} \in \mathscr{L}(M)$. Then the distribution $I_{M_{1}}(\pi, X, f)=I_{\mathfrak{b}}(\pi, X, f)$ equals

$$
\int_{i \mathfrak{a}_{M, S}^{*} / i \mathfrak{a}_{M_{1}, S}^{*}} I_{M_{1}}\left(\pi_{\lambda}, h_{M_{1}}(X), f\right) e^{-\lambda(X)} d \lambda,
$$

an absolutely convergent integral.

Corollary 8.5. Given $\pi \in \Pi\left(M\left(F_{S}\right)\right)$ and $X \in \mathfrak{a}_{M, S}$, we have

$$
I_{M_{1}}(\pi, X, f)=\sum_{L \in \mathscr{L}(M)} d_{M}^{G}\left(M_{1}, L\right) \widehat{I}_{M}^{L}\left(\pi, X, f_{L}\right), \quad f \in \mathscr{H}_{\mathrm{ac}}\left(G\left(F_{S}\right)\right) .
$$

Suppose that $\mathfrak{b}$ is a proper subspace of $\mathfrak{a}_{M}$. Then $d_{M}^{G}(\mathfrak{b}, L)$ is nonzero only when $L \neq G$, in which case the distributions $\widehat{I}_{M}^{L}$ are all well defined. Strictly speaking, the two theorems are only valid for such $\mathfrak{b}$. However, until we complete the induction in the next paper [1(f)], it will be understood that $\widehat{I}\left(f_{G}\right)$ 
really means $I(f)$, for any given invariant distribution $I$ on $\mathscr{H}_{\text {ac }}\left(G\left(F_{S}\right)\right)$. With this temporary abuse of notation, the theorem and corollaries of this paragraph are all valid as stated.

\section{SPLitTing}

The splitting properties are essentially special cases of Theorems 8.1 and 8.4. However, they are important enough to discuss separately on their own. To state them, we take $S$ to be the disjoint union of two sets $S_{1}$ and $S_{2}$. We assume that both $S_{1}$ and $S_{2}$ have the closure property. Theorem 11.1 of [1(a)] provides a splitting formula for $I_{M}(\gamma)$ that applies to elements $\gamma \in M\left(F_{S}\right)$ which are $G$-regular. We must generalize it to arbitrary elements in $M\left(F_{S}\right)$.

Proposition 9.1. Suppose that

$$
\gamma=\gamma_{1} \gamma_{2}, \quad \gamma_{i} \in M\left(F_{S_{i}}\right),
$$

is any element in $M\left(F_{S}\right)$. Then for any function $f \in \mathscr{H}_{\mathrm{ac}}\left(G\left(F_{S}\right)\right)$ of the form

$$
f=f_{1} f_{2}, \quad f_{i} \in \mathscr{H}_{\mathrm{ac}}\left(G\left(F_{S_{i}}\right)\right),
$$

we have

$$
I_{M}(\gamma, f)=\sum_{L_{1}, L_{2} \in \mathscr{L}(M)} d_{M}^{G}\left(L_{1}, L_{2}\right) \widehat{I}_{M}^{L_{1}}\left(\gamma_{1}, f_{1, L_{1}}\right) \widehat{I}_{M}^{L_{2}}\left(\gamma_{2}, f_{2, L_{2}}\right) .
$$

Proof. This is essentially a special case of Theorem 8.1. We say essentially because we must in fact replace $(G, M)$ by the pair $(\mathscr{G}, \mathscr{M})=(G \times G, M \times M)$, in which the products are regarded as varieties over the ring $F \times F$. However, the definitions of $\S 8$ extend in a straightforward way to this setting. We take $b$ to be the space $\mathfrak{a}_{M}$, embedded diagonally in $\mathfrak{a}_{\mathscr{M}}=\mathfrak{a}_{M} \oplus \mathfrak{a}_{M}$. Notice that

$$
G\left(F_{S}\right)=(G \times G)\left(F_{S_{1}} \times F_{S_{2}}\right)=\mathscr{G}\left((F \times F)_{S_{1} \times S_{2}}\right) .
$$

It follows without difficulty that

$$
I_{b}(\gamma, f)=2^{(1 / 2) \operatorname{dim}\left(\mathfrak{a}_{M}^{G}\right)} I_{M}\left(\gamma_{1} \gamma_{2}, f_{1} f_{2}\right) .
$$

Obviously $M_{\mathfrak{b}}=M \times M=\mathscr{M}$, so that $\gamma_{1} \gamma_{2}=\left(\gamma_{1} \gamma_{2}\right)^{\mathfrak{b}}$. As we noted in the discussion prior to Corollary $7.4, \mathscr{L}(\mathscr{M})$ is the set of pairs $\mathscr{L}=\left(L_{1}, L_{2}\right)$, $L_{i} \in \mathscr{L}(M)$. Clearly

$$
\widehat{I}_{\mathscr{M}}^{\mathscr{L}}\left(\gamma, f_{\mathscr{L}}\right)=\widehat{I}_{M}^{L_{1}}\left(\gamma_{1}, f_{1, L_{1}}\right) \widehat{I}_{M}^{L_{2}}\left(\gamma_{2}, f_{2, L_{2}}\right)
$$

(As in the proof of Theorem 8.1, this really comes from the distribution $I_{\mathscr{M}}^{\mathscr{L}}\left(\gamma, f_{Q_{\mathscr{L}}}\right)$, so there is no intrinsic difficulty with the notation $\widehat{I}_{\mathscr{M}}^{\mathscr{L}}$, even in the case $L_{i}=G$ where our induction hypothesis does not apply.) Since

$$
d_{\mathscr{M}}^{\mathscr{G}}(\mathfrak{b}, L)=2^{(1 / 2) \operatorname{dim}\left(\mathfrak{a}_{M}^{G}\right)} d_{M}^{G}\left(L_{1}, L_{2}\right),
$$

Theorem 8.1 gives the required formula for $I_{M}(\gamma, f)$. 
Remarks. (1) If we combine Proposition 9.1 with Corollary 8.2, we obtain the formula

$$
I_{M}(\gamma, f)=\sum_{L \in \mathscr{L}(M)} \widehat{I}_{M}^{L}\left(\gamma_{1}, f_{1, L}\right) I_{L}\left(\gamma_{2}^{L}, f_{2}\right) .
$$

This was actually the splitting formula derived in Theorem 11.1 of [1(a)] in the special case of $\gamma$ regular.

(2) According to the induction assumption of $\S 2$, the Fourier transform $\widehat{I}_{M}^{L_{i}}\left(\gamma_{i}\right)$ is defined if $L_{i} \varsubsetneqq G$. However,

$$
d_{M}^{G}(M, G)=d_{M}^{G}(G, M)=1,
$$

sc there are terms in the formula of the proposition with $L_{i}=G$. For these terms, it is understood that

$$
\widehat{I}_{G}\left(\gamma_{i}, f_{G}\right)=I_{G}\left(\gamma_{i}, f\right),
$$

as we agreed at the end of $\S 8$.

It is sometimes useful to combine the splitting and descent properties into one formula. Suppose that for each $v \in S, M_{v}$ is a Levi subset of $M$ which is defined over $F_{v}$. We can of course apply all our earlier definitions with $F$ replaced by $F_{v}$. In particular, we have the real vector space $\mathfrak{a}_{M_{v}}$, and the map

$$
H_{M_{v}}: M_{v}\left(F_{v}\right) \rightarrow \mathfrak{a}_{M_{v}}
$$

We should point out that even if $M_{v}$ equals $M$, the spaces $\mathfrak{a}_{M_{v}}$ and $\mathfrak{a}_{M}$ need not be equal, for they are defined relative to the different fields $F_{v}$ and $F$. Set $\mathscr{M}=\prod_{v \in S} M_{v}$ and $\mathfrak{a}_{\mathscr{M}}=\bigoplus_{v \in S} \mathfrak{a}_{M_{v}}$. If we think of $\mathscr{M}$ as a Levi subset of $M$ defined over $F_{S}$, it will be clear how to extend our earlier definitions. For example, $\mathscr{L}(\mathscr{M})$ will denote the set of

$$
\mathscr{L}=\prod_{v \in S} L_{v}, \quad L_{v} \in \mathscr{L}\left(M_{v}\right) .
$$

Given such an $\mathscr{L}$, we can define the distribution

$$
I_{\mathscr{M}}^{\mathscr{L}}(\gamma), \quad \gamma \in \mathscr{M}\left(F_{S}\right),
$$

on $\mathscr{H}_{\mathrm{ac}}\left(\mathscr{L}\left(F_{S}\right)\right)$, and the map $f \rightarrow f_{\mathscr{L}}$ from $\mathscr{H}_{\mathrm{ac}}\left(G\left(F_{S}\right)\right)$ to $\mathscr{I}_{\mathrm{ac}}\left(\mathscr{L}\left(F_{S}\right)\right)$. We also have a constant $d_{\mathscr{M}}^{G}(M, \mathscr{L})$. It is defined to be zero unless the natural map

$$
\mathfrak{a}_{\mathscr{M}}^{M} \oplus \mathfrak{a}_{\mathscr{M}}^{\mathscr{L}} \rightarrow \mathfrak{a}_{\mathscr{M}}^{G}
$$

is an isomorphism, in which case $d_{\mathscr{M}}^{G}(M, \mathscr{L})$ is the volume in $\mathfrak{a}_{\mathscr{M}}^{G}$ of the parallelogram generated by orthonormal bases of $\mathfrak{a}_{\mathscr{M}}^{M}$ and $\mathfrak{a}_{\mathscr{M}}^{\mathscr{L}}$.

Corollary 9.2. Suppose that $\gamma=\prod_{v \in S} \gamma_{v}$ is a point in $\mathscr{M}\left(F_{S}\right)$, and that

$$
\gamma^{M}=\prod_{v \in S} \gamma_{v}^{M}
$$


is the induced space in $M\left(F_{S}\right)$. Then

$$
I_{M}\left(\gamma^{M}, f\right)=\sum_{\mathscr{L} \in \mathscr{L}(\mathscr{M})} d_{\mathscr{M}}^{G}(M, \mathscr{L}) \widehat{I}_{\mathscr{M}}^{\mathscr{L}}\left(\gamma, f_{\mathscr{L}}\right),
$$

for any function $f \in \mathscr{H}_{\mathrm{ac}}\left(G\left(F_{S}\right)\right)$.

Proof. It is easy to see how to extend Theorem 8.1 in a formal way so that it includes Corollary 9.2 as well as Proposition 9.1 as special cases. Alternatively, the corollary follows by repeatedly applying Theorem 8.1 and Proposition 9.1 directly.

Remarks. In the special case that $\gamma$ is regular, a similar formula was stated in [1(a), Corollary 11.3]. However, the proof there does not apply in the generality claimed. For in $[1(\mathrm{a})]$ we failed to account for the fact that the space $\mathfrak{a}_{M}$ depends on the ground field over which $M$ is taken. Theorem 12.1 of [1(a)] is likewise affected, for it depends on Corollary 11.3 of [1(a)]. As established in [1(a)], these results are only valid of $G$ is an inner twist of a split group. We hasten to add, however, that $\S \S 11$ and 12 of [1(a)] have since been subsumed in other results, and are no longer needed. For example, Theorem 12.1 of [1(a)] can be replaced by the assertion that $\phi_{M}$ maps $\mathscr{H}_{\text {ac }}\left(G\left(F_{S}\right)\right)$ continuously to $\mathscr{I}_{\text {ac }}\left(M\left(F_{S}\right)\right)$. This was established as Theorem 12.1 of $[1(\mathrm{e})]$. It can also be proved quite simply by applying Corollary 7.2 directly to the $(G, M)$-family from which $\phi_{M}(f, \pi, X)$ is defined. However, the proof in [1(e)] has the advantage of providing an obstruction, in terms of residues, for a function $\phi_{M}(f)$ to lie in $\mathscr{J}\left(M\left(F_{S}\right)\right)$.

Corollary 9.3. For each $v \in S$, set $M_{v}=M$, and suppose that the distributions

$$
I_{M_{v}}^{L_{v}}\left(\gamma_{v}\right), \quad L_{v} \in \mathscr{L}\left(M_{v}\right), \gamma_{v} \in M_{v}\left(F_{v}\right)
$$

are supported on characters. Then the corresponding distributions

$$
I_{M}^{L}(\gamma), \quad L \in \mathscr{L}(M), \gamma \in M\left(F_{S}\right),
$$

for $F_{S}$ are also supported on characters. In particular, the induction assumption of $\S 2$ is valid for $(G / F, S)$, provided that it holds for each $\left(G / F_{v},\{v\}\right)$.

Proof. We need only consider the case that $L=G$. Fix $\gamma \in M\left(F_{S}\right)$. We must show that $I_{M}(\gamma)$ annihilates the functions $f \in \mathscr{H}\left(G\left(F_{S}\right)\right)$ such that $f_{G}=0$. We leave the reader to check that any such function can be approximated by linear combinations of functions of the form

$$
\prod_{v \in S} f_{v}, \quad f_{v} \in \mathscr{H}\left(G\left(F_{v}\right)\right),
$$

in which $f_{w, G}=0$ for some valuation $w$ in $S$. Corollary 9.2 tells us that $I_{M}(\gamma)$ vanishes on this latter function.

The splitting formula for the dual distributions is similar. Let

$$
\pi=\pi_{1} \otimes \pi_{2}, \quad \pi_{i} \in \Pi\left(M\left(F_{S_{i}}\right)\right),
$$


be an arbitrary representation in $\Pi\left(M\left(F_{S}\right)\right)$, and consider a point

$$
X=\left(X_{1}, X_{2}\right), \quad X_{i} \in \mathfrak{a}_{M, S_{i}} .
$$

For each $f \in \mathscr{H}_{\text {ac }}\left(G\left(F_{S}\right)\right)$, we shall write

$$
J_{M}(\pi, X, f)=\int J_{M}\left(\pi_{\lambda}, X_{1}+X_{2}, f\right) e^{-\lambda(X)} d \lambda
$$

and

$$
I_{M}(\pi, X, f)=\int I_{M}\left(\pi_{\lambda}, X_{1}+X_{2}, f\right) e^{-\lambda(X)} d \lambda,
$$

where each integral is taken over the direct sum of $i \mathfrak{a}_{M, S_{1}}^{*}$ and $i \mathfrak{a}_{M, S_{2}}^{*}$, modulo the diagonally embedded image of $i \mathrm{a}_{M, S}^{*}$. Both integrals converge absolutely, and we have

$$
I_{M}(\pi, X, f)=J_{M}(\pi, X, f)-\sum_{L \in \mathscr{L}_{0}(M)} \widehat{I}_{M}^{L}\left(\pi, X, \phi_{L}(f)\right) .
$$

Specializing Theorem 8.4 , we obtain

Proposition 9.4. Let $\pi=\pi_{1} \otimes \pi_{2}$ and $X=\left(X_{1}, X_{2}\right)$ be as above. Then for any function

we have

$$
f=f_{1} f_{2}, \quad f_{i} \in \mathscr{H}_{\mathrm{ac}}\left(G\left(F_{S_{i}}\right)\right)
$$

$$
I_{M}(\pi, X, f)=\sum_{L_{1}, L_{2} \in \mathscr{L}(M)} d_{M}^{G}\left(L_{1}, L_{2}\right) \widehat{I}_{M}^{L_{1}}\left(\pi_{1}, X_{1}, f_{1, L_{1}}\right) \widehat{I}_{M}^{L_{2}}\left(\pi_{2}, X_{2}, f_{2, L_{2}}\right) .
$$

Remark. Proposition 9.4, and also the results Theorem 8.4 and Corollary 8.5 of the last section, have obvious analogues if $\pi$ is replaced by a standard representation $\rho \in \Sigma\left(M\left(F_{S}\right)\right)$.

\section{THE EXAMPLE OF $G L(n)$. LOCAL VANishing PROPERTIES}

Let us look at an example. We shall show that for $G L(n)$, the invariant distributions sometimes vanish. These vanishing results, which extend those of $\S 14$ of [1(a)], demonstrate how the descent formula of $\S 8$ can be usefully applied. They will also be needed in the study of base change for $G L(n)$.

The first lemma is a companion to Lemma 14.1 of [1(a)]. Together, the two results summarize the algebraic properties of $G L(n)$ that are behind the vanishing results.

Lemma 10.1. Suppose that $G=G L(n)$. Let $L, L_{1}$, and $L_{2}$ be Levi subgroups of $G$ over $F$, with $L_{1} \subset L$ and $L_{1} \subset L_{2}$, such that $d_{L_{1}}^{G}\left(L, L_{2}\right) \neq 0$. Then the natural map

is surjective.

$$
X(L)_{F} \oplus X\left(L_{2}\right)_{F} \rightarrow X\left(L_{1}\right)_{F}
$$

Proof. Fix an isomorphism

$$
L_{1} \stackrel{\sim}{\longrightarrow} G L\left(n_{1}\right) \times \cdots \times G L\left(n_{r}\right) .
$$


If

is an arbitrary point in $L_{1}$, set

$$
x \rightarrow x_{1} \times \cdots \times x_{r}, \quad x_{i} \in G L\left(n_{i}\right),
$$

$$
\chi_{i}(x)=\operatorname{det}\left(x_{i}\right), \quad 1 \leq i \leq r .
$$

Then $\left\{\chi_{i}: 1 \leq i \leq r\right\}$ is a basis of $X\left(L_{1}\right)_{F}$. Once the isomorphism above is fixed, the group $L \in \mathscr{L}\left(L_{1}\right)$ corresponds canonically to a partition of the set $\{1, \ldots, r\}$ into disjoint subsets $S_{1}, \ldots, S_{p}$. The characters

$$
\prod_{i \in S_{j}} \chi_{i}, \quad 1 \leq j \leq p
$$

form a basis of $X(L)_{F}$. Similarly, $L_{2}$ corresponds to a partition of $\{1, \ldots, r\}$ into disjoint subsets $T_{1}, \ldots, T_{q}$. We must show that each $\chi_{i}$ belongs to $X(L)_{F} \oplus X\left(L_{2}\right)_{F}$.

The nonvanishing of $d_{L_{1}}^{G}\left(L, L_{2}\right)$ is equivalent to the property that

$$
\mathfrak{a}_{L}^{*} \oplus \mathfrak{a}_{L_{2}}^{*} \rightarrow \mathfrak{a}_{L_{1}}^{*},
$$

is a surjective, with 1-dimensional kernel $\left\{(Z,-Z): Z \in \mathfrak{a}_{G}^{*}\right\}$. The reader can check that this implies (a) that $p+q=r+1$, and (b) that no proper nonempty subset of $\{1, \ldots, r\}$ is a simultaneous union of sets $S_{j}$ or $T_{k}$. According to (a), one of the two partitions contains a set consisting of one element. To be definite, we can assume that $S_{p}=\{r\}$. Then the character $\chi_{r}$ belongs to $X(L)_{F} \oplus X\left(L_{2}\right)_{F}$. The element $r$ also belongs to a unique set $T_{k}$, and (b) implies that $T_{k}$ contains more than one element. In other words, $T_{k}^{\prime}=T_{k}-\{r\}$ is not empty. We obtain two disjoint partitions $S_{1}, \ldots, S_{p-1}$ and $T_{1}, \ldots, T_{k}^{\prime}, \ldots, T_{q}$ of the set $\{1, \ldots, r-1\}$, which also satisfy (a) and (b). Since the character $\prod_{i \in T_{k}^{\prime}}^{q} \chi_{i}$ belongs to $X(L)_{F} \oplus X\left(L_{2}\right)_{F}$, the lemma follows by induction on $r$.

For the rest of this section we shall assume that we have been given an inner twist

$$
\eta: G \rightarrow G^{*}=(G L(n) \times \cdots \times G L(n)) \rtimes \theta^{*},
$$

as in (1.2). We shall let $E$ denote the smallest extension of $F$ over which the image of the cocycle

$$
\eta^{\sigma} \eta^{-1}, \quad \sigma \in \operatorname{Gal}(\bar{F} / F)
$$

in $G^{+} / G^{0}$ splits. Then $E$ is a cyclic extension of $F$ whose degree $l_{E}$ over $F$ divides $l$. This is just the setup for base change of a central simple algebra. One can show that

$$
G^{0}(F) \cong \underbrace{G L\left(\frac{n}{d}, D \otimes E\right) \times \cdots \times G L\left(\frac{n}{d}, D \otimes E\right)}_{l_{1}}, \quad l_{1}=l l_{E}^{-1},
$$

where $d$ is a divisor of $n$, and $D$ is a division algebra of degree $d^{2}$ over $F$. 
We shall write $G^{\prime}$ for the group $G L(n)$, embedded diagonally in $\left(G^{*}\right)^{0}$. We are going to show that our invariant distributions on $G^{\prime}$ vanish on certain data related to $G$, in a sense that depends only on the integer $d$ and the field $E$. Suppose that $L$ is a Levi subgroup of $G^{\prime}$ (defined over $F$ ). As in [1(a)], we write

$$
\mathfrak{p}(L)=\left(n_{1}, \ldots, n_{r}\right), \quad n_{1} \geq n_{2} \geq \cdots \geq n_{r},
$$

for the unique partition of $n$ such that

$$
L \cong G L\left(n_{1}\right) \times \cdots \times G L\left(n_{r}\right) .
$$

We shall say that $L$ comes from $G$ if $d$ divides each of the integers $n_{i}$. This means that there is a Levi subset $M$ of $G$ such that $L=M^{\prime}$. In other words, $L$ is embedded diagonally in $\left(M^{*}\right)^{0}$, where $M^{*}$ is some product of components of the form (1.1) which is related to $M$ by inner twisting. Suppose that $L_{1} \subset L_{2}$ are two other Levi subgroups of $G^{\prime}$ with $d_{L_{1}}^{G^{\prime}}\left(M^{\prime}, L_{2}\right) \neq 0$. Then if $L_{2}$ comes from $G$, Lemma 14.1 of $[1(\mathrm{a})]$ asserts that $L_{1}$ also comes from $G$.

Recall that an element $\delta \in G^{\prime}(F)$ is $F$-elliptic if it lies in a maximal torus of $G^{\prime}$ which is anisotropic over $F$, modulo $A_{G}$. We shall write $G^{\prime}(F)_{\text {ell }}$ for the set of such elements. By the theory of elementary divisors every çonjugacy class in $G^{\prime}(F)$ is induced from an elliptic class. In other words, for any $\delta \in G^{\prime}(F)$ there is a Levi subgroup $L_{1}$ of $G^{\prime}$, and an element $\tau \in L_{1}(F)_{\text {ell }}$, such that $\delta$ belongs to the induced conjugacy class $\tau^{G^{\prime}}$. The pair $\left(L_{1}, \tau\right)$ is uniquely determined by $\delta$ up to $G^{\prime}(F)$-conjugacy. We shall say that $\delta$ comes from $G$ if the group $L_{1}$ comes from $G$, and if for every character $\xi_{1} \in X\left(L_{1}\right)_{F}$, the element $\xi_{1}(\tau)$ belongs to $N_{E / F}\left(E^{*}\right)$, the image of the norm from $E^{*}$. We shall write $G^{\prime}(F)_{G}$ for the set of such elements. We shall also write $G^{\prime}(F)^{G}$ simply for the set of elements $\delta \in G^{\prime}(F)$ such that $\xi(\delta)$ belongs to $N_{E / F}\left(E^{*}\right)$ for any $\xi \in X(G)_{F}$. Then $G^{\prime}(F)_{G}$ is a subset of $G^{\prime}(F)^{G}$. Observe that if $M^{\prime}$ is a Levi subset of $G^{\prime}$ which comes from $G$, we can also define the subsets $M^{\prime}(F)_{M} \subset M^{\prime}(F)^{M}$ of $M^{\prime}(F)$.

Suppose now that $F$ is a local field and that $S=\{v\}$, so that $F=F_{v}=F_{S}$. Let $f^{\prime}$ be a fixed function in $\mathscr{H}\left(G^{\prime}(F)\right)$ such that

$$
I_{G^{\prime}}\left(\zeta, f^{\prime}\right)=0
$$

for any $G^{\prime}$-regular element $\zeta \in G^{\prime}(F)$ which does not belong to $G^{\prime}(F)_{G}$.

Proposition 10.2. Suppose that $M^{\prime}$ is a Levi subgroup of $G^{\prime}$ which comes from $G$ and that $\delta$ belongs to $M^{\prime}(F)^{M}$. Then $I_{M^{\prime}}\left(\delta, f^{\prime}\right)=0$, unless $\delta$ lies in $M^{\prime}(F)_{M}$.

Remark. If $M^{\prime}=G^{\prime}$, the proposition is essentially a restatement of the definition of $f^{\prime}$. It is of course the case $M^{\prime} \neq G^{\prime}$ that is interesting.

Proof. Assume that $I_{M^{\prime}}\left(\delta, f^{\prime}\right) \neq 0$. Fix a pair

$$
\left(L_{1}, \tau\right), \quad \tau \in L_{1}(F)_{\mathrm{ell}}, \delta \in \tau^{M^{\prime}},
$$


and a character $\xi_{1} \in X\left(L_{1}\right)_{F}$. We must show that $L_{1}$ comes from $M$ and that $\xi_{1}(\tau)$ belongs to $N_{E / F}\left(E^{*}\right)$.

The situation is made to order for our descent formula. For Corollary 8.2 immediately yields an expansion

$$
I_{M^{\prime}}\left(\delta, f^{\prime}\right)=\sum_{L_{2} \in \mathscr{L}\left(L_{1}\right)} d_{L_{1}}^{G^{\prime}}\left(M^{\prime}, L_{2}\right) \widehat{I}_{L_{1}}^{L_{2}}\left(\tau, f_{L_{2}}^{\prime}\right)
$$

and hence the existence of some $L_{2} \in \mathscr{L}\left(L_{1}\right)$ with

$$
d_{L_{1}}^{G}\left(M^{\prime}, L_{2}\right) \widehat{I}_{L_{1}}^{L_{2}}\left(\tau, f_{L_{2}}^{\prime}\right) \neq 0
$$

The nonvanishing of $d_{L_{1}}^{G^{\prime}}\left(M^{\prime}, L_{2}\right)$ allows us to apply Lemma 10.1. We obtain

$$
\xi_{1}=\xi+\xi_{2}, \quad \xi \in X\left(M^{\prime}\right)_{F}, \xi_{2} \in X\left(L_{2}\right)_{F} .
$$

Now the distribution $\hat{I}_{L_{1}}^{L_{2}}\left(\tau, f_{L_{2}}^{\prime}\right)$ belongs to the closed linear span of $\left\{\hat{I}_{L_{2}}^{L_{2}}\left(\zeta, f_{L_{2}}^{\prime}\right)\right\}$, where $\zeta$ ranges over the $G$-regular points in $L_{2}(F)$ with $\xi_{2}(\tau)=$ $\xi_{2}(\zeta)$. But $\widehat{I}_{L_{1}}^{L_{2}}\left(\tau, f_{L_{2}}^{\prime}\right)$ does not vanish, so there exists such a $\zeta$ with

$$
\widehat{I}_{L_{2}}^{L_{2}}\left(\zeta, f_{L_{2}}^{\prime}\right)=I_{G}\left(\zeta, f^{\prime}\right) \neq 0 .
$$

It follows from the definition of $f^{\prime}$ that $L_{2}$ comes from $G$ and that $\xi_{2}(\tau)$ belongs to $N_{E / F}\left(E^{*}\right)$. Applying Lemma 14.1 of [1(a)], we see that $L_{1}$ also comes from $G$. This obviously implies that $L_{1}$ comes from $M$, our first required condition. Moreover, by assumption, the element $\xi(\tau)=\xi(\delta)$ belongs to $N_{E / F}\left(E^{*}\right)$. Therefore, the element $\xi_{1}(\tau)=\xi(\tau) \xi_{2}(\tau)$ also belongs to $N_{E / F}\left(E^{*}\right)$. This is the second required condition.

There is a parallel vanishing property for the distributions

$$
I_{M^{\prime}}\left(\pi, Y, f^{\prime}\right), \quad \pi \in \Pi\left(M^{\prime}(F)\right), Y \in \mathfrak{a}_{M^{\prime}, v} .
$$

We shall only deal with the first half of it here. The other half will appear as Lemma II.8.1 of [2].

Proposition 10.3. Suppose that $M^{\prime}$ is a Levi subgroup of $G^{\prime}$ which comes from $G$ and that $L_{1}$ is a Levi subgroup of $M^{\prime}$. Then

$$
I_{M^{\prime}}\left(\pi, Y, f^{\prime}\right)=0
$$

for any $Y \in \mathfrak{a}_{M^{\prime}, v}$, and any induced representation

$$
\pi=\pi_{1, \lambda}^{M^{\prime}}, \quad \lambda \in i \mathfrak{a}_{L, v}^{*}, \pi_{1} \in \Pi\left(L_{1}(F)\right),
$$

unless $L_{1}$ comes from $M$.

Proof. The proof is similar to that of the last proposition. It is enough to show that if $L_{1}$ does not come from $M$, then the Fourier transform

$$
I_{M^{\prime}}\left(\pi_{1}, Y_{1}, f^{\prime}\right)=\int_{i a_{L_{1}, v}^{*} / i a_{M^{\prime}, v}^{*}} I_{M^{\prime}}\left(\pi_{1, \lambda}^{M^{\prime}}, Y_{1}, f^{\prime}\right) e^{-\lambda\left(Y_{1}\right)} d \lambda
$$


vanishes, for every point $Y_{1} \in \mathfrak{a}_{L_{1}, v}$ whose projection onto $\mathfrak{a}_{M^{\prime}, v}$ equals $Y$. The descent formula, Corollary 8.5 , yields

$$
I_{M^{\prime}}\left(\pi_{1}, Y_{1}, f^{\prime}\right)=\sum_{L_{2} \in \mathscr{L}\left(L_{1}\right)} d_{L_{1}}^{G^{\prime}}\left(M^{\prime}, L_{2}\right) \hat{I}_{L_{1}}^{L_{2}}\left(\pi_{1}, Y_{1}, f_{L_{2}}^{\prime}\right) .
$$

The proposition then follows as above from [1(a), Lemma 14.1].

Remark. Obviously, a similar vanishing property holds if $\pi$ and $\pi_{1}$ are replaced by standard representations $\rho \in \Sigma\left(M^{\prime}(F)\right)$ and $\rho_{1} \in \Sigma\left(L_{1}(F)\right)$.

The function $f^{\prime}$ is intended to come from a function on $G(F)$ by a transfer of orbital integrals. To make this more plausible, we shall describe the set $G^{\prime}(F)_{G}$ in terms of the norm mapping from $G(F)$ to $G^{\prime}(F)$. This discussion is not really needed here but will be used in the article [2] (in combination with $\S \mathrm{I} .2$ of that paper).

We shall first recall some elementary facts, for which $F$ can be a general field. Any element

$$
y=\left(y_{1}, \ldots, y_{l}\right) \rtimes \theta^{*}
$$

in $G^{*}$ is $\left(G^{*}\right)^{0}$-conjugate to the point

$$
\left(1, \ldots, 1, y_{1} \cdots y_{l}\right) \rtimes \theta^{*} .
$$

Consequently, $y^{l}$ is $\left(G^{*}\right)^{0}$-conjugate to an element in $G^{\prime}$, which is uniquely determined up to $G^{\prime}$-conjugacy. We obtain a bijection from the $\left(G^{*}\right)^{0}$-orbits in $G^{*}$ onto the conjugacy classes in $G^{\prime}$. A given orbit in $G^{*}$ meets $G^{*}(F)$ if and only if the corresponding conjugacy class in $G^{\prime}$ meets $G^{\prime}(F)$.

Suppose that $\gamma$ belongs to $G(F)$. For any $\sigma \in \operatorname{Gal}(\bar{F} / F)$, we have

$$
\eta(\gamma)^{\sigma}=\eta^{\sigma}\left(\gamma^{\sigma}\right)=\left(\eta^{\sigma} \eta^{-1}\right)(\eta(\gamma)) \text {. }
$$

By assumption, $\eta^{\sigma} \eta^{-1}$ is an inner automorphism of $\left(G^{*}\right)^{+}$. Since the centralizer of $\eta(\gamma)$ intersects $G^{*}$, the points $\eta(\gamma)^{\sigma}$ and $\eta(\gamma)$ are $\left(G^{*}\right)^{0}$-conjugate. Thus, $\operatorname{Gal}(\bar{F} / F)$ preservec the $\left(G^{*}\right)^{0}$-orbit of $\eta(\gamma)$. Equivalently, $\operatorname{Gal}(\bar{F} / F)$ preserves the $G^{\prime}$-orbit of $(\eta(\gamma))^{l}$. It follows from the theory of elementary divisors that the $G^{\prime}$-conjugacy class of $\eta(\gamma)^{l}$ has a representative in $G^{\prime}(F)$. The same is therefore true of the $\left(G^{*}\right)^{0}$-orbit of $\eta(\gamma)$. In other words, there is an element $c_{\gamma}$ in $\left(G^{*}\right)^{0}$ such that the point $\gamma^{*}=c_{\gamma} \eta(\gamma) c_{\gamma}^{-1}$ belongs to $G^{*}(F)$. One can, in fact, assume that $\gamma^{*}$ is of the form

$$
\left(1, \ldots, \gamma^{\prime}\right) \rtimes \theta^{*}, \quad \gamma^{\prime} \in G L_{n}(F) .
$$

Then the element

$$
\gamma^{\prime}=\left(\gamma^{*}\right)^{l}=c_{\gamma} \eta(\gamma)^{l} c_{\gamma}^{-1}=\left(\gamma^{\prime}, \ldots, \gamma^{\prime}\right)
$$

belongs to $G^{\prime}(F)$ and is uniquely determined up to $G^{\prime}(F)$-conjugacy. The correspondence $\gamma \rightarrow \gamma^{\prime}$ gives a map from $G^{0}(F)$-orbits in $G(F)$ into $G^{\prime}(F)$ conjugacy classes, which is easily seen to be injective. This is the norm from 
$G(F)$ to $G^{\prime}(F)$. The symbol $\gamma^{\prime}$ can denote either a conjugacy class or some element in the class.

If $\gamma$ is as above, the function

$$
\eta_{\gamma}(x)=c_{\gamma} \eta(x) c_{\gamma}^{-1}, \quad x \in G^{+},
$$

maps $G_{\gamma}$ onto $G_{\gamma^{*}}^{*}$. But $\gamma^{*}$ is of the form (10.2), and one sees immediately that $G_{\gamma^{*}}$ equsls $G_{\gamma^{\prime}}^{\prime}$. Therefore, $\eta_{\gamma}$ is an isomorphism from $G_{\gamma}$ onto $G_{\gamma^{\prime}}^{\prime}$. It follows easily from the definitions that it is actually an inner twist. Now, suppose that $\sigma \in G(F)$ is semisimple. Then the group $G_{\sigma}$, together with the inner twist, $\eta_{\sigma}: G_{\sigma} \rightarrow G_{\sigma^{\prime}}^{\prime}$, satisfies our original conditions on $G$ (with $l=1$ ). We shall denote the corresponding norm mapping from conjugacy classes $\{\mu\}$ in $G_{\sigma}(F)$ to conjugacy classes $\left\{c_{\mu} \eta_{\sigma}(\mu) c_{\mu}^{-1}\right\}$ in $G_{\sigma^{\prime}}^{\prime}(F)$ by $\mu \rightarrow \mu_{\sigma^{\prime}}$. If

$$
\gamma=\sigma \mu, \quad \mu \in G_{\sigma}(F),
$$

one can take $c_{\gamma}=c_{\mu} c_{\sigma}$, and one obtains

$$
\mu^{\prime}=\sigma^{\prime} \mu_{\sigma^{\prime}}^{l} .
$$

We return to the case that $F$ is a local field.

Lemma 10.4. The image of the norm map is $G^{\prime}(F)_{G}$. In other words, $G^{\prime}(F)_{G}$ is the union over all $\gamma \in G(F)$ of the conjugacy classes $\gamma^{\prime}$.

Proof. Suppose that $\delta$ is an arbitrary element in $G^{\prime}(F)$. Then $\delta \in \tau^{G}$, where $\tau \in L_{1}(F)_{\text {ell }}$ for a Levi subgroup $L_{1}$ of $G^{\prime}$. This means that $\delta$ lies in the conjugacy class of $\tau \nu$, where $\nu$ belongs to the Richardson orbit in $G_{\tau}^{\prime}$ corresponding to the Levi subgroup $L_{1, \tau}$. Suppose that $\delta$ equals the norm of an element $\gamma \in G(F)$ with Jordan decomposition $\gamma=\sigma u$. Then (10.3) yields $\gamma^{\prime}=\sigma^{\prime} u_{\sigma^{\prime}}^{l}$, which is just the Jordan decomposition of $\gamma^{\prime}$. We can therefore assume that $\tau=\sigma^{\prime}$ and $\nu=u_{\sigma^{\prime}}^{l}$. Now $u_{\sigma^{\prime}}^{l}$ is conjugate in $G_{\sigma^{\prime}}^{\prime}(F)$ to the element $u_{\sigma^{\prime}}$. In other words, the inner twist

$$
\eta_{\sigma}: G_{\sigma} \rightarrow G_{\sigma^{\prime}}^{\prime}=G_{\tau}^{\prime}
$$

maps $u$ to the Richardson orbit in $G_{\tau}^{\prime}$ corresponding to $L_{1, \tau}$. It follows that $L_{1, \tau}$ is the image of a Levi subgroup of $G_{\sigma}$ over $F$. But any such subgroup will necessarily be of the form $M_{1, \sigma}$, where $M_{1}$ is a Levi subset of $G$ over $F$ which contains $\sigma$. Moreover $\eta_{\sigma}: M_{1} \rightarrow L_{1}$ is an inner twist with respect to which $\tau$ is the norm of $\sigma$. Conversely, given $\delta=\tau \nu$, suppose that $L_{1}$ comes from a Levi subset $M_{1}$ of $G$ and that $\tau$ is the norm of an element $\sigma \in M_{1}(F)$. Working backwards, we see that $\delta$ is the norm of an element $\gamma=\sigma u$ in $G(F)$.

We have obtained a reduction of the proof. We have only to establish, for any $L_{1}=M_{1}^{\prime}$ which comes from $G$, and any elliptic element $\tau \in L_{1}(F)$, that $\tau$ belongs to $L_{1}(F)^{M_{1}}$ if and only if $\tau=\sigma^{\prime}$ for some element $\sigma \in M_{1}(F)$. We may assume that $L_{1}=G^{\prime}$ and $M_{1}=G$. 
One way is quite formal. Let $G_{a b}$ be the quotient of $G$ by the derived subgroup of $G^{0}$. Then $G_{a b}$ is a component which satisfies the same hypothesis as $G$. Writing $\{G(F)\}$ in general for the set of $G^{0}(F)$-orbits in $G(F)$, we embed the norm map $\{G(F)\} \rightarrow\left\{G^{\prime}(F)\right\}$ in a commutative diagram

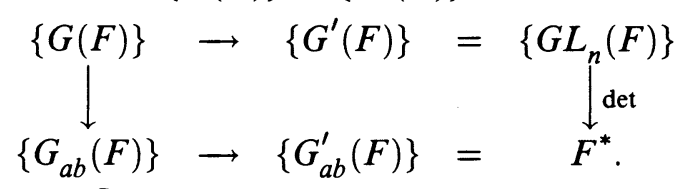

The subset $G^{\prime}(F)^{G}$ of $G^{\prime}(F)$ consists of those elements whose image in $F^{*}$ lies in the subgroup $N_{E / F}\left(E^{*}\right)$. But

$$
G_{a b}(F) \cong \underbrace{\left(E^{*} \times \cdots \times E^{*}\right)}_{l_{1}} \rtimes \theta_{1}, \quad l_{1}=l l_{E}^{-1},
$$

where $\theta_{1}$ is an automorphism

$$
\left(y_{1}, \ldots, y_{l_{1}}\right) \rightarrow\left(y_{2}, \ldots, y_{l_{1}}, \sigma\left(y_{1}\right)\right), \quad y_{i} \in E^{*},
$$

for a fixed generator $\sigma$ of $\operatorname{Gal}(E / F)$. The lower horizontal arrow in the diagram can be identified with the map

$$
\left(y_{1}, \ldots, y_{l_{1}}\right) \rtimes \theta_{1} \rightarrow N_{E / F}\left(y_{1}\right) \cdots N_{E / F}\left(y_{l_{1}}\right), \quad y_{i} \in E^{*} .
$$

It follows that any element in $G^{\prime}(F)$ which is a norm from $G(F)$ lies in $G^{\prime}(F)^{G}$.

Conversely, suppose that $\tau$ is an $F$-elliptic element (relative to $G$ ) in $G^{\prime}(F)^{G}$. Then $\tau \in T^{\prime}(F)$, where $T^{\prime}$ is a maximal torus in $G^{\prime}$ over $F$ which is anisotropic modulo $A_{G}$. Fix an isomorphism $T^{\prime}(F) \cong F_{1}^{*}$, where $F_{1} / F$ is an extension of degree $n$. Then the restriction of the determinant to $T^{\prime}(F)$ is identified with $N_{F_{1} / F}$. The theory of simple algebras attaches a maximal torus $T^{0}$ of $G^{0}$ to the algebra $E_{1}=E \otimes_{F} F_{1}$. In fact, there is a subgroup $T^{+}$of $G^{+}$over $F$, such that

$$
T(F)=T^{+}(F) \cap G(F) \cong \underbrace{\left(E_{1}^{*} \times \cdots \times E_{1}^{*}\right)}_{l_{1}} \rtimes \theta_{1},
$$

where $\theta_{1}$ is an automorphism

$$
\left(u_{1}, \ldots, u_{l_{1}}\right) \rightarrow\left(u_{2}, \ldots, u_{l_{1}}, \sigma\left(u_{1}\right)\right), \quad u_{i} \in E_{1}^{*} .
$$

Here $\sigma$ is the automorphism of $E_{1} / F_{1}$ determined by a generator of $\operatorname{Gal}(E / F)$. It follows that there is an element $c_{\tau} \in G$ such that

$$
t \rightarrow c_{\tau} \eta(t)^{l} c_{\tau}^{-1}, \quad \tau \in T(F)
$$

the restriction of the norm to $T(F)$, corresponds to the map

$$
\left(u_{1}, \ldots, u_{l_{1}}\right) \rtimes \theta \rightarrow \prod_{i=1}^{l_{1}} N_{E_{1} / F_{1}}\left(u_{i}\right), \quad u_{i} \in E_{1}^{*} .
$$


It is an exercise in local class field theory to show that the image of this map is the subgroup

$$
\left\{y \in F_{1}^{*}: N_{F_{1} / F}(y) \in N_{E / F}\left(E^{*}\right)\right\},
$$

of $F_{1}^{*}$. (See Lemma I.1.4 of [2].) It follows that $\tau$ equals $\sigma^{\prime}$, for some element $\sigma \in G(F)$. This completes the proof of the lemma.

\section{APPENDIX. CONVEX POLYTOPES}

Let $\mathfrak{a}$ be a finite dimensional Euclidean space. A convex polytope $\Pi$ in $\mathfrak{a}$ is the convex hull of a finite set of points. Fix such a $\Pi$, and let $\mathscr{F}(\Pi)$ denote the finite set of closed faces of $\Pi$. Then $\mathscr{F}(\Pi)$ is a partially ordered set whose elements are convex polytopes in their own right. The maximal element is just $\Pi$, while the minimal elements form the subset $\mathscr{P}(\Pi)$ of faces which are just points. The faces in $\mathscr{P}(\Pi)$ are of course called the vertices of $\Pi$. Suppose that $F$ is a face in $\mathscr{F}(\Pi)$. The (open) dual cone $\mathfrak{a}_{F}^{+}$is defined as follows. Choose a point $X_{F}$ in $F$ which does not lie on any proper subface of $F$ and form the cone generated by $\Pi-X_{F}$. Then $\mathfrak{a}_{F}^{+}$is the relative interior of the corresponding dual cone. That is, $\mathfrak{a}_{F}^{+}$is the intersection, over all points $X$ in the complement of $F$ in $\Pi$, of the half open spaces

$$
\left\{Y \in \mathfrak{a}:\left\langle Y, X-X_{F}\right\rangle<0\right\} .
$$

Let $\mathfrak{a}^{F}$ denote the subspace of $\mathfrak{a}$ spanned by $F-X_{F}$, and let $\mathfrak{a}_{F}$ be the orthogonal complement of $\mathfrak{a}^{F}$ in $\mathfrak{a}$. Then $\mathfrak{a}_{F}^{+}$is an open convex cone in $\mathfrak{a}_{F}$ which is independent of $X_{F}$.

It is a basic fact that $\mathfrak{a}$ is the disjoint union of the cones $\mathfrak{a}_{F}^{+}$. Let us recall how this is proved. The dual cones consist of cosets of $\mathfrak{a}_{\Pi}$ and are invariant under translation of $\Pi$. We may therefore assume that $\Pi$ contains the origin as an interior point. Let $\widehat{\Pi}$ be the polar set of $\Pi[4, \S \S 6,9]$. More precisely, $\widehat{\Pi}$ is the intersection, over all points $X \in \Pi$, of the closed half spaces

$$
\{Y \in \mathfrak{a}:\langle Y, X\rangle \leq 1\} \text {. }
$$

Then $\hat{\Pi}$ is another convex polytope, whose interior contains the origin. There is an incidence reversing bijection $F \leftrightarrow \widehat{F}$ between the proper faces of $\Pi$ and $\widehat{\Pi}$, and $\mathfrak{a}_{F}^{+}$is just the cone generated by the relative interior of $\widehat{F}$. But any half line through the origin will intersect the relative interior of a unique proper face $\widehat{F}$. Therefore, $\mathfrak{a}$ is indeed a disjoint union of the cones $\mathfrak{a}_{F}^{+}$.

Suppose that $\mathfrak{b}$ is a vector subspace of $\mathfrak{a}$, and let $\Pi_{\mathfrak{b}}$ be the projection of $\Pi$ onto $\mathfrak{b}$. Then $\Pi_{\mathfrak{b}}$ is also a convex polytope. We shall construct a section from $\Pi_{\mathfrak{b}}$ into $\Pi$. We must first fix a point $\xi$ in $\mathfrak{a}^{\mathfrak{b}}$, the orthogonal complement of $\mathfrak{b}$ in $\mathfrak{a}$, which is in general position. Let $\mathscr{F}(\Pi, \xi)$ denote the set of faces $F \in \mathscr{F}(\Pi)$ for which the set

$$
\mathfrak{b}_{\xi, F}=(\xi+\mathfrak{b}) \cap \mathfrak{a}_{F}^{+}
$$


is not empty. Then $(\xi+\mathfrak{b})$ is a disjoint union over $\mathscr{F}(\Pi, \xi)$ of the sets $\mathfrak{b}_{\xi, F}$. Define

$$
\Pi(\xi)=\bigcup_{F \in \mathscr{F}(\Pi, \xi)} F
$$

The general position of $\xi$ implies that if $F$ belongs to $\mathscr{F}(\Pi, \xi)$ and if $F_{1} \in$ $\mathscr{F}(\Pi)$ is a face which is contained in $F$, then $F_{1}$ also belongs to $\mathscr{F}(\Pi, \xi)$. It follows that $\Pi(\xi)$ is a subcomplex of $\Pi$.

Lemma A.1. The orthogonal projection of $\mathfrak{a}$ onto $\mathfrak{b}$ maps $\Pi(\xi)$ bijectively onto $\Pi_{\mathrm{b}}$.

Proof. Let $\eta$ be a point in $\Pi_{b}$. The fiber at $\eta$ is the set

$$
\Pi^{\eta}=\Pi \cap\left(\eta+\mathfrak{a}^{b}\right) .
$$

We must show that $\Pi^{\eta}$ intersects $\Pi(\xi)$ at precisely one point.

The faces of $\Pi(\xi)$ are the elements in $\mathscr{F}(\Pi, \xi)$. Observe that $\mathscr{F}(\Pi, \xi)$ is the subset of faces $F \in \mathscr{F}(\Pi)$ such that $\xi$ belongs to $\left(\mathfrak{a}_{F}^{+}+\mathfrak{b}\right)$. On the other hand, $\Pi^{\eta}$ is also a convex polytope, and its faces are of the form

$$
F^{\eta}=F \cap \Pi^{\eta}, \quad F \in \mathscr{F}(\Pi) .
$$

Many of these intersections will be empty. Moreover, if $\eta$ is not in general position, different $F$ will give the same intersection. However, let us define $\mathscr{F}^{\eta}(\Pi)$ to be the set of elements $F \in \mathscr{F}(\Pi)$ such that $F^{\eta}$ contains a point $X_{F}^{\eta}$ in the relative interior of $F$. Any such $F$ will be minimal among those faces which have the same intersection with $\Pi^{\eta}$. Clearly $F \rightarrow F^{\eta}$ is a bijection from $\mathscr{F}^{\eta}(\Pi)$ onto the set of faces of $\Pi^{\eta}$.

Suppose that $F \in \mathscr{F}^{\eta}(\Pi)$ and that $F_{1} \in \mathscr{F}(\Pi)$ is some other face such that $F_{1}^{\eta}=F^{\eta}$. Then $\mathfrak{a}^{F} \cap \mathfrak{a}^{\mathfrak{b}}=\mathfrak{a}^{F_{1}} \cap \mathfrak{a}^{\mathfrak{b}}$. Taking orthogonal complements, we obtain $\mathfrak{a}_{F}+\mathfrak{b}=\mathfrak{a}_{F_{1}}+\mathfrak{b}$. However, $F$ is minimal, so it is actually a face of $F_{1}$. This means that $\mathfrak{a}_{F_{1}}^{+}$is contained in the closure of $\mathfrak{a}_{F}^{+}$. It follows easily that

$$
\mathfrak{a}_{F}^{+}+\mathfrak{b} \supseteq \mathfrak{a}_{F_{1}}^{+}+\mathfrak{b} .
$$

Thus, in studying the intersection of $\Pi(\xi)$ with $\Pi^{\eta}$, we need only consider those faces of $\Pi(\xi)$ which belong to $\mathscr{F}^{\eta}(\Pi)$. Set

Suppose again that $F \in \mathscr{F}^{\eta}(\Pi)$. We shall find the dual cone $\mathfrak{a}_{F^{\eta}}^{+}$of $F^{\eta}$.

and

$$
C_{F}=\left\{t\left(X-X_{F}^{\eta}\right): t \geq 0, X \in \Pi\right\}
$$

$$
C_{F^{\eta}}=\left\{t\left(X^{\eta}-X_{F}^{\eta}\right): t \geq 0, X^{\eta} \in \Pi^{\eta}\right\} .
$$

Then $C_{F}^{\eta}=C_{F} \cap \mathfrak{a}^{\mathfrak{b}}$. But $C_{F}$ and $\mathfrak{a}^{\mathfrak{b}}$ are both polyhedral cones. As is well known, the dual cone of their intersection equals the sum of their dual cones. It follows that the closure of $\mathfrak{a}_{F \eta}^{+}$equals the sum of the closure of $\mathfrak{a}_{F}^{+}$with $\mathfrak{b}$. Taking the relative interior of these closed cones, we obtain

$$
\mathfrak{a}_{F^{\eta}}^{+}=\mathfrak{a}_{F}^{+}+\mathfrak{b} .
$$


We know that $\mathfrak{a}$ is the disjoint union of the cones $\mathfrak{a}_{F^{\eta}}^{+}$. We can therefore express $\mathfrak{a}$ as the disjoint union, over $F \in \mathscr{F}^{\eta}(\Pi)$, of the cones $\mathfrak{a}_{F}^{+}+\mathfrak{b}$. In particular, $\xi$ lies in precisely one such cone. But $\xi$ is in general position, so we can assume that the cone in which it lies is open and corresponds to a vertex of $\Pi^{\eta}$. We have thus shown that there is precisely one face of $\Pi^{\eta}$ which meets $\Pi(\xi)$ and that this face is a vertex. In other words, $\Pi^{\eta}$ meets $\Pi(\xi)$ in precisely one point, as required.

Our purpose in discussing convex polytopes has of course been for their connection with $(G, M)$-families. Let us consider a typical example. For each $Q \in \mathscr{F}(M)$, let $\rho_{Q} \in \mathfrak{a}_{Q}^{+}$be the usual vector defined by the square root of the modular function. Let $\Pi_{M}$ denote the convex hull of the finite set $\left\{\rho_{P}: P \in \mathscr{P}(M)\right\}$. Then $\Pi_{M}$ is a convex polytope which lies in $\mathfrak{a}_{M}^{G}$. There is an order preserving bijection $Q \rightarrow \Pi_{M}^{Q}$ from $\mathscr{F}(M)$ onto the set of faces of $\Pi_{M}$. Moreover, the dual cone of $\Pi_{M}^{Q}$ is just the chamber $\mathfrak{a}_{Q}^{+}$. Thus, the face $\Pi_{M}^{Q}$ and the chamber $\mathfrak{a}_{Q}^{+}$are of complementary dimensions, and they intersect orthogonally at the point $\rho_{Q}$. Consider the $(G, M)$-family given by

$$
c_{P}(\lambda)=e^{\lambda\left(\rho_{P}\right)}, \quad P \in \mathscr{P}(M), \lambda \in i \mathfrak{a}_{M}^{*} .
$$

Then $c_{M}(\lambda)$ is just the integral of $e^{-\lambda(H)}$ over $\Pi_{M}$. (See $\S 6$ of [1(a)].) More generally, suppose that $Q \in \mathscr{F}(M)$. Then $\Pi_{M}^{Q}$ lies in the affine space $\rho_{Q}+\mathfrak{a}_{M}^{Q}$ and inherits a Euclidean measure $d H$ from that on $\mathfrak{a}_{M}^{Q}$. We have

$$
c_{M}^{Q}(\lambda)=\int_{\Pi_{M}^{Q}} e^{-\lambda(H)} d H, \quad \lambda \in \mathfrak{a}_{M, \mathcal{C}}^{*} .
$$

In particular, $c_{M}^{Q}$ is just the volume in $\rho_{Q}+\mathfrak{a}_{M}^{Q}$ of the face $\Pi_{M}^{Q}$.

Now, as in $\S 7$, suppose that $\mathfrak{b}$ is a special subspace of $\mathfrak{a}_{M}$. Let $\Pi_{M, \mathfrak{b}}$ be the projection of $\Pi_{M}$ onto $\mathfrak{b}$. We claim that the $\left(\mathfrak{a}_{G}, \mathfrak{b}\right)$-family associated to $\left\{c_{P}(\lambda)\right\}$ is also the one attached to the polytope $\Pi_{M, \mathfrak{b}}$. If $\mathfrak{p}$ is any element in $\mathscr{P}(\mathfrak{b})$, let $Q$ be the unique element in $\mathscr{P}\left(M_{\mathfrak{b}}\right)$ such that $\mathfrak{b}_{\mathfrak{p}}^{+}$is contained in $\mathfrak{a}_{Q}^{+}$and define $\rho_{\mathrm{p}}$ to be the projection of $\rho_{Q}$ onto $\mathfrak{b}$. Then

$$
c_{\mathfrak{p}}(\nu)=e^{\nu\left(\rho_{\mathfrak{p}}\right)}, \quad \mathfrak{p} \in \mathscr{P}(\mathfrak{b}), \nu \in i \mathfrak{b}^{*},
$$

is the associated $\left(\mathfrak{a}_{G}, \mathfrak{b}\right)$-family. On the other hand, $\Pi_{M, \mathfrak{b}}$ is the convex hull in $\mathfrak{b}$ of the set

$$
\left\{\rho_{\mathfrak{p}}: \mathfrak{p} \in \mathscr{P}(\mathfrak{b})\right\} .
$$

For it is trivial that $\Pi_{M, b}$ contains the convex hull. The converse is a minor extension of Lemma 3.1 of [1(b)j and is proved the same way. Our claim, then, is justified. In particular, as in $\S 6$ of [1(a)], we can write

$$
c_{\mathfrak{b}}(\nu)=\int_{\Pi_{M, b}} e^{-\nu(\widetilde{H})} d \widetilde{H}, \quad \nu \in i \mathfrak{b}^{*},
$$

where $d \widetilde{H}$ is the Euclidean measure on $\mathfrak{b}$. 
We shall want to apply Lemma A.1. As before, let $\xi$ be a point in $\mathfrak{a}_{M}^{\mathfrak{b}}$ in general position and write $\mathscr{F}(M, \xi)$ for the set of elements $Q \in \mathscr{F}(M)$ such that the set

is not empty. Then

$$
\mathfrak{b}_{\xi, Q}=(\xi+\mathfrak{b}) \cap \mathfrak{a}_{Q}^{+}
$$

$$
\xi+\mathfrak{b}=\bigcup_{Q \in \mathscr{F}(M, \xi)} \mathfrak{b}_{\xi, Q}
$$

is a decomposition of $\xi+\mathfrak{b}$ into a polyhedral complex. The vertices correspond to the parabolics $Q_{L}$ introduced in $\S 7$. The maximal cells correspond to the set

$$
\mathscr{P}(M, \xi)=\mathscr{P}(M) \cap \mathscr{F}(M, \xi) .
$$

We note that $\mathscr{P}(M, \xi)$ is just the set of $P \in \mathscr{P}(M)$ which are contained in one of the parabolics $Q_{L}$. Of particular interest are the cells which are translates in $\xi+\mathfrak{b}$ of the chambers $\mathfrak{b}_{\mathfrak{p}}^{+}$in $\mathfrak{b}$. Let us write $\mathscr{P}_{\text {ext }}(M, \xi)$ for the subset of elements $P \in \mathscr{P}(M, \xi)$ such that the closure of $\mathfrak{a}_{P}^{+}$intersects $\mathfrak{b}$ in an open set. This intersection must necessarily be the closure of a chamber $\mathfrak{b}_{\mathfrak{p}(P)}^{+}$ for a uniquely determined element $\mathfrak{p}(P)$ in $\mathscr{P}(\mathfrak{b})$. We claim that the map $P \rightarrow \mathfrak{p}(P)$ is a bijection from $\mathscr{P}_{\text {ext }}(M, \xi)$ onto $\mathscr{P}(\mathfrak{b})$. For suppose that $\mathfrak{p}$ is an arbitrary element in $\mathscr{P}(\mathfrak{b})$. Let $Q$ be the unique element in $\mathscr{P}\left(M_{\mathfrak{b}}\right)$ such that $\mathfrak{a}_{Q}^{+}$contains $\mathfrak{a}_{P}^{+}$and let $R$ be the unique element in $\mathscr{P}^{M_{\mathfrak{b}}}(M)$ such that $\xi$ belongs to $\mathfrak{a}_{R}^{+}$. Then $P=Q(R)$ is the unique element in $\mathscr{P}_{\text {ext }}(M, \xi)$ with $\mathfrak{p}(P)=\mathfrak{p}$. We point out that $\rho_{\mathfrak{p}(P)}$ is just the projection of $\rho_{P}$ onto $\mathfrak{b}$.

We will use Lemma A.1 to study the function

$$
c_{\mathfrak{b}}(\nu), \quad \nu \in i \mathfrak{b}^{*} \text {. }
$$

Observe that the maximal cells in the complex

$$
\Pi_{M}(\xi)=\bigcup_{Q \in \mathscr{F}(M, \xi)} \Pi_{M}^{Q}
$$

correspond to the parabolics $Q_{L}$, where $L$ ranges over the elements in $\mathscr{L}(M)$ with $d_{M}^{G}(\mathfrak{b}, L) \neq 0$. Let $\widetilde{\Pi}_{M}^{Q_{L}}$ be the projection of $\Pi_{M}^{Q_{L}}$ onto $\mathfrak{b}$. Then Lemma A.1 asserts that $\Pi_{M, b}$ is the disjoint union of the sets $\widetilde{\Pi}_{M}^{Q_{L}}$, together with a set of measure 0 . It follows from (A.3) that

$$
c_{\mathfrak{b}}(\nu)=\int_{\Pi_{M, \mathfrak{b}}} e^{-\nu(\widetilde{H})} d \widetilde{H}=\sum_{L} \int_{\widetilde{\Pi}_{M}^{Q_{L}}} e^{-\nu(\widetilde{H})} d \widetilde{H} .
$$

Fix $L$ for the moment and let $H \rightarrow \widetilde{H}$ denote the orthogonal projection of $\mathfrak{a}_{M}^{L}$ onto $\mathfrak{b}^{G}$. We are assuming that $d_{M}^{G}(\mathfrak{b}, L) \neq 0$, so that this map is an isomorphism, and $d \widetilde{H}=d_{M}^{G}(\mathfrak{b}, L) d H$. Moreover, $e^{-\nu(H)}=e^{-\nu(\widetilde{H})}$, since $\nu$ belongs to $i \mathfrak{b}^{*}$. It follows that

$$
\int_{\widetilde{\Pi}_{M}^{Q_{L}}} e^{-\nu(\widetilde{H})} d \widetilde{H}=d_{M}^{G}(\mathfrak{b}, L) \int_{\Pi_{M}^{Q_{L}}} e^{-\nu(H)} d H
$$


Combining these formulas with (A.2), we obtain

$$
c_{\mathfrak{b}}(\nu)=\sum_{L \in \mathscr{L}(M)} d_{M}^{G}(\mathfrak{b}, L) c_{M}^{Q_{L}}(\nu), \quad \nu \in i \mathfrak{b}^{*} .
$$

On the other hand, we have

$$
\begin{aligned}
c_{\mathfrak{b}}(\nu) & =\sum_{\mathfrak{p} \in \mathscr{P}(\mathfrak{b})} e^{\nu\left(\rho_{\mathfrak{p}}\right)} \theta_{\mathfrak{p}}(\nu)^{-1} \\
& =\sum_{P \in \mathscr{P}_{\operatorname{Pxl}}(M, \xi)} e^{\nu\left(\rho_{P}\right)} \theta_{\mathfrak{p}(P)}(\nu)^{-1}
\end{aligned}
$$

from our correspondence between $\mathscr{P}_{\text {ext }}(M, \xi)$ and $\mathscr{P}(\mathfrak{b})$. It thus follows that

$$
\sum_{L \in \mathscr{L}(M)} d_{M}^{G}(\mathfrak{b}, L) c_{M}^{Q_{L}}(\nu)=\sum_{P \in \mathscr{P}_{\text {exl }}(M, \xi)} e^{\nu\left(\rho_{P}\right)} \theta_{\mathfrak{p}(P)}(\nu)^{-1},
$$

for any point $\nu \in i \mathfrak{b}^{*}$ and for $\left\{c_{P}(\lambda)\right\}$ the $(G, M)$-family given by (A.1).

Our ultimate purpose has been to prove Proposition 7.1. We can at last do this. Suppose that $\left\{c_{P}(\lambda)\right\}$ is an arbitrary $(G, M)$-family. The expression

$$
\sum_{L \in \mathscr{L}(M)} d_{M}^{G}(\mathfrak{b}, L) c_{M}^{Q_{L}}(\lambda)
$$

equals

$$
\sum_{L \in \mathscr{L}(M)} d_{M}^{G}(\mathfrak{b}, L) \sum_{\left\{P \in \mathscr{P}(M): P \subset Q_{L}\right\}} c_{P}(\lambda) \theta_{P \cap L}(\lambda)^{-1} .
$$

Let $r_{P, \xi}(\lambda)$ denote the sum, over all elements $L \in \mathscr{F}(M)$ with $d_{M}^{G}(\mathfrak{b}, L) \neq 0$ and with $Q_{L} \supset P$, of the terms

$$
d_{M}^{G}(\mathfrak{b}, L) \theta_{P \cap L}(\lambda)^{-1} .
$$

Then

$$
\sum_{L \in \mathscr{L}(M)} d_{M}^{G}(\mathfrak{b}, L) c_{M}^{Q_{L}}(\lambda)=\sum_{P \in \mathscr{P}(M, \xi)} c_{P}(\lambda) r_{P, \xi}(\lambda)
$$

Set $\lambda$ equal to a point $\nu$ in $i b^{*}$, and for the moment take $\left\{c_{P}(\nu)\right\}$ to be the $(G, M)$-family defined by (A.1). Then we can combine (A.5) with (A.4). We obtain

$$
\sum_{P \in \mathscr{P}_{\mathrm{cxl}}(M, \xi)} e^{\nu\left(\rho_{P}\right)} \theta_{\mathfrak{p}(P)}(\nu)^{-1}=\sum_{P \in \mathscr{P}(M, \xi)} e^{\nu\left(\rho_{P}\right)} r_{P, \xi}(\nu) .
$$

The functions $\left\{\theta_{\mathfrak{p}(P)}(\nu)^{-1}\right\}$ and $\left\{r_{P, \xi}(\nu)\right\}$ are all rational in $\nu$. Furthermore, by Lemma A.1, the projection of the set

$$
\left\{\rho_{P}: P \in \mathscr{P}(M, \xi)\right\}
$$

onto $\mathfrak{b}$ is injective. Therefore the exponential functions

$$
\nu \rightarrow e^{\nu\left(\rho_{P}\right)}, \quad P \in \mathscr{P}(M, \xi),
$$


are linearly independent over the field of rational functions. Setting the coefficients equal to 0 , we find that

$$
r_{P, \xi}(\nu)=\left\{\begin{array}{l}
\theta_{\mathfrak{p}(P)}(\nu)^{-1}, \quad \text { if } P \in \mathscr{P}_{\text {ext }}(M, \xi), \\
0, \quad \text { otherwise. }
\end{array}\right.
$$

Returning to the case that $\left\{c_{P}(\lambda)\right\}$ is arbitrary, we substitute the formula for $r_{P, \xi}(\nu)$ into the right-hand side of (A.5). We obtain

$$
\begin{aligned}
\sum_{L \in \mathscr{L}(M)} d_{M}^{G}(\mathfrak{b}, L) c_{M}^{Q_{L}}(\nu) & =\sum_{P \in \mathscr{P}_{\text {exl }}(M, \xi)} c_{P}(\nu) \theta_{\mathfrak{p}(P)}(\nu)^{-1} \\
& =\sum_{\mathfrak{p} \in \mathscr{P}(\mathfrak{b})} c_{\mathfrak{p}}(\nu) \theta_{\mathfrak{p}}(\nu)^{-1} \\
& =c_{\mathfrak{b}}(\nu) .
\end{aligned}
$$

This completes the proof of Proposition 7.1.

\section{BIBLIOGRAPHY}

1. J. Arthur, (a) The trace formula in invariant form, Ann. of Math. 114 (1981), 1-74.

(b) On a family of distributions obtained from Eisenstein series II: Explicit formulas, Amer. J. Math. 104 (1982), 1289-1336.

(c) On a family of distributions obtained from orbits, Canad. J. Math. 38 (1986), 179-214.

(d) The local behaviour of weighted orbital integrals, Duke Math. J. 56 (1988), 1-71.

(e) Intertwining operators and residues I. Weighted characters, J. Funct. Anal. (to appear).

(f) The invariant trace formula II. Global theory, J. Amer. Math. Soc. (to appear).

2. J. Arthur and L. Clozel, Base change for $G L(n)$, Ann. of Math. Stud. (to appear).

3. J. Bernstein, P. Deligne, and D. Kazhdan, Trace Paley-Wiener theorem for reductive $p$-adic groups, preprint.

4. A. Brønsted, An introduction to convex polytopes, Graduate Texts in Mathematics, SpringerVerlag, New York, 1983.

5. L. Clozel and P. Delorme, (a) Le théorème de Paley-Wiener invariant pour les groupes de Lie réductifs, Inv. Math. 77 (1984), 427-453.

(b) Sur le théorème de Paley-Wiener invariant pour les groupes de Lie réductifs réels, C. $\mathrm{R}$. Acad. Sci. Paris. Sér. I Math., t. 300, 11 (1985), 331-333.

6. L. Clozel, J.-P. Labesse, and R. Langlands, Morning seminar on the trace formula, Lecture Notes, Institute for Advanced Study, Princeton Univ.

7. R. Langlands, On the functional equations satisfied by Eisenstein series, Lecture Notes in Math., Springer, Berlin and New York, no. 544, 1976.

8. J. Rogawski, The trace Paley-Wiener theorem in the twisted case, preprint.

9. D. Shelstad, L-indistinguishability for real groups, Math. Ann. 259 (1982), 385-430.

Max-Planck-Institut für Mathmatik, Gottfried-Claren-Str. 26, D-5300 Bonn 3, FedERAL REPUBLIC OF GERMANY

Department of Mathematics, University of Toronto, Toronto, Canada, M5S 1A1 